Hope College

Hope College Digital Commons

Faculty Publications

7-16-2013

\title{
VPT2+K Spectroscopic Constants and Matrix Elements of the Transformed Vibrational Hamiltonian of a Polyatomic Molecule with Resonances Using Van Vleck Perturbation Theory
}

Andreana M. Rosnik

Hope College

William F. Polik

Hope College, polik@hope.edu

Follow this and additional works at: https://digitalcommons.hope.edu/faculty_publications

Part of the Atomic, Molecular and Optical Physics Commons, and the Chemistry Commons

\section{Recommended Citation}

Repository citation: Rosnik, Andreana M. and Polik, William F., "VPT2+K Spectroscopic Constants and Matrix Elements of the Transformed Vibrational Hamiltonian of a Polyatomic Molecule with Resonances Using Van Vleck Perturbation Theory" (2013). Faculty Publications. Paper 1066.

https://digitalcommons.hope.edu/faculty_publications/1066

Published in: Molecular Physics, Volume 112, Issue 2, July 16, 2013, pages 261-300. Copyright (c 2013

Taylor \& Francis, Milton Park, Abingdon.

This Article is brought to you for free and open access by Hope College Digital Commons. It has been accepted for inclusion in Faculty Publications by an authorized administrator of Hope College Digital Commons. For more information, please contact digitalcommons@hope.edu. 
VPT2+K spectroscopic constants and matrix elements of the transformed vibrational Hamiltonian of a polyatomic molecule with resonances using Van Vleck perturbation theory

Andreana M. Rosnik and William F. Polik*

Department of Chemistry, Hope College, Holland, MI, United States

Andreana M. Rosnik, Department of Chemistry, Hope College, United States, andreana.rosnik@hope.edu.

*William F. Polik, Department of Chemistry, Hope College, 35 East $12^{\text {th }}$ Street, Holland, MI 49422-9000, United States, 616-395-7639 (telephone), 616-395-7118 (fax), polik@ hope.edu. 


\section{VPT2+K spectroscopic constants and matrix elements of the transformed vibrational Hamiltonian of a polyatomic molecule with resonances using Van Vleck perturbation theory}

Vibrational levels of polyatomic molecules are analyzed with Van Vleck perturbation theory to connect experimental energy levels to computed molecular potential energy surfaces. Vibrational matrix elements are calculated from a quartic potential function via second order Van Vleck perturbation theory, a procedure that treats both weak and strong interactions among vibrational states by approximately block-diagonalizing the vibrational Hamiltonian. A clear and complete derivation of anharmonic and resonance constants as well as general expressions for both on- and off-diagonal matrix elements of the transformed Hamiltonian is presented. The equations are written in partial fraction form and as a constant multiplied by a harmonic oscillator matrix element to facilitate removing the effect of strongly interacting resonant states both in analytical formulae and in computer code. The derived equations are validated numerically, and results for the isotopomers of formaldehyde $\left(\mathrm{H}_{2} \mathrm{CO}, \mathrm{HDCO}\right.$, $\mathrm{D}_{2} \mathrm{CO}$ ) are included. The implications of the equations on zero-point energy calculations and experimental fits are discussed. The VPT $2+\mathrm{K}$ method is defined by these results for use in fitting and calculating vibrational energy levels.

Keywords: molecular vibrations; Van Vleck perturbation theory; vibrational energy

levels; spectroscopic constants; resonances 


\section{Introduction}

Chemists use potential energy surfaces (PES's) to understand chemical structure and reactivity. These potential surfaces are of dimension $3 N-6$, the number of internal degrees of freedom for nonlinear polyatomic molecules, and are functions of the internal coordinates of the molecule, e.g., the bond lengths and angles of the molecule. PES's are mathematical constructs and therefore cannot be directly measured. However, changes in bond lengths and angles correspond to molecular vibrations. Observation of molecular vibrational energy levels provides the best experimental insight into PES's because different vibrational modes probe different internal coordinates and increasing numbers of vibrational quanta probe further away from the equilibrium geometry.

PES's are generated in two general ways. Theoretically, the potential function itself is computed using ab initio calculations or experimental parameters. Experimentally, vibrational energy levels are probed using spectroscopy, and the general shape of the potential wells can be inferred from these energy levels. In this work, an approach connecting experiment and theory is implemented, by which a potential function is used to derive expressions for vibrational energy levels and for spectroscopic constants beyond vibrational frequencies $\omega_{i}$, such as anharmonic constants $x_{k l}$ and resonance constants $K$. The resulting equations are useful for predicting experimental energy levels and assessing methods for computing potentials by comparing calculated and observed vibrational levels. While the $\omega_{i}$ connect experimental and computed second order derivatives, i.e., harmonic force constants, the $x_{k l}$ and $K$ constants are the most direct link between experimental and computed higher order force constants.

Other attempts to relate spectroscopic constants to potential constants have appeared in the literature [1-6]. However, they are presented without derivation [3, 6], with error in the 
derivation $[1,4]$, with omission of some resonance constants $[3,5-6]$, as primarily numerical methods [7], or with error in the final results [3-4]. Furthermore, the contributions of neglected zero-point terms are not addressed $[1,3,4,6]$, which are crucial in applying electronic structure theory to experimental situations.

In polyatomic molecules, many small interactions (perturbations) and some large interactions (resonances) are observed, creating a multi-scale problem involving a wide variety of interaction strengths. When present, the strong interactions among vibrational modes greatly affect vibrational spectra. Resonances give rise to level shifting and mixing. Their exclusion from calculations can lead to poor fits and inaccurate predictions. As resonances result in strong interactions, they cannot be treated using standard second order perturbation theory (PT2). Consequently, in the derivation of spectroscopic constants and matrix elements of the transformed vibrational Hamiltonian presented in this work, second order Van Vleck perturbation theory (VVPT) is used. The method implemented here is called VPT2 $+\mathrm{K}$, for second order vibrational perturbation theory with resonances. VVPT allows for the treatment of both perturbations and resonances simultaneously. Furthermore, VVPT leads to general matrix elements for vibrational interactions. This manuscript presents a clear and complete derivation using a quartic potential energy surface to relate potential constants to vibrational energy levels. Furthermore, expressions for matrix elements of the transformed vibrational Hamiltonian, as well as anharmonic and resonance constants for second order resonances, are derived and presented.

In addition to the derivation and results, the formulae in this work have been implemented and tested numerically. Computational implementation and verification of the formulae were performed, and examples are presented to illustrate the effectiveness of the expressions and programs. The results include the calculation of vibrational energy levels and spectroscopic constants for three example cases of molecules with resonances - 
formaldehyde $\left(\mathrm{H}_{2} \mathrm{CO}\right)$, singly-deuterated formaldehyde (HDCO), and doubly-deuterated formaldehyde $\left(\mathrm{D}_{2} \mathrm{CO}\right)$. Practical applications of the equations are discussed, including calculation of zero-point energies, fitting of experimental data, and implementation of the resulting formulae in computer code.

Section II of this paper defines the Hamiltonian, force constants, and non-restrictive summations used throughout the paper. Section III outlines the Van Vleck transformation and presents the derivation of general second order Van Vleck perturbation theory matrix elements. Using these general matrix elements, section IV.A details the derivation of ondiagonal matrix elements of the transformed vibrational Hamiltonian, and section IV.B presents the derivation of the various types of off-diagonal matrix elements. Section V discusses practical points about the implementation of these formulae, such as the effect of resonances on anharmonic and resonance constants, the partial fraction decomposition of matrix element expressions to isolate resonance denominators, and the removal of resonance terms from sets of strongly interacting states. Section VI describes the computer implementation of the equations and presents their numerical verification. Finally, section VII applies the coded expressions to the isotopomers of formaldehyde and compares the results to experiment. The key expressions that define the VPT2+K computational method are presented in Tables 2 and 3.

\section{The vibrational Hamiltonian}

The vibration-rotation Hamiltonian of a nonlinear polyatomic molecule was expressed by Watson [8] as

$$
\hat{H}_{v i b-r o t}=\frac{1}{2} \sum_{\alpha \beta} \mu_{\alpha \beta}\left(\hat{J}_{\alpha}-\hat{\pi}_{\alpha}\right)\left(\hat{J}_{\beta}-\hat{\pi}_{\beta}\right)-\frac{\hbar^{2}}{8} \sum_{\alpha} \mu_{\alpha \alpha}+\frac{1}{2} \sum_{k=1}^{3 N-6} \hat{P}_{k}^{2}+V
$$

The vibration-only part of Equation (1) is 


$$
\hat{H}_{v i b}=\frac{1}{2} \sum_{\alpha \beta} \mu_{\alpha \beta} \hat{\pi}_{\alpha} \hat{\pi}_{\beta}-\frac{\hbar^{2}}{8} \sum_{\alpha} \mu_{\alpha \alpha}+\frac{1}{2} \sum_{k=1}^{3 N-6} \hat{P}_{k}^{2}+V
$$

In Equations (1) and (2), $\mu_{\alpha \beta}$ is the inertia tensor, $\hat{J}_{\alpha}$ the total angular momentum operator, $\hat{\pi}_{\alpha}$ the vibrational angular momentum operator, $\hat{P}_{k}$ the momentum operator, and $V$ the potential expressed in normal coordinates $\hat{Q}_{k} . \alpha$ and $\beta$ are rotational axis indices, and $k$ is a normal vibrational mode index. $\hat{P}_{k}$ is defined as $\frac{\partial \hat{Q}_{k}}{\partial t}$. In Equation (2), the first term is vibrational angular momentum, the second term is a small constant with no contribution to transition energies at second order, the third term is the part of kinetic energy associated with the harmonic vibration, and the fourth term is the potential energy. Consequently, the Hamiltonian used in this work is defined as

$$
\hat{H}=\frac{1}{2} \sum_{\alpha \beta} \mu_{\alpha \beta} \hat{\pi}_{\alpha} \hat{\pi}_{\beta}+\frac{1}{2} \sum_{k=1}^{3 N-6} \hat{P}_{k}^{2}+V
$$

Since $V$ is a function of normal coordinates $\hat{Q}_{k}$, which are referenced to the equilibrium geometry, the leading kinetic and potential terms in Equation (2) are given by the harmonic oscillator Hamiltonian

$$
\hat{H}^{o}=\frac{1}{2} \sum_{k=1}^{3 N-6}\left(\hat{P}_{k}^{2}+\lambda_{k} \hat{Q}_{k}^{2}\right)
$$

where $\lambda_{k}=\left(2 \pi c \omega_{k}\right)^{2}, c$ is the speed of light, and $\omega_{k}$ is the harmonic vibrational frequency in wavenumbers.

To express Equation (4) in unitless operators, the dimensionless normal coordinate and momentum operators $q_{k}$ and $p_{k}$ are introduced

$$
q_{k}=\left(\frac{\lambda_{k}}{\hbar^{2}}\right)^{\frac{1}{4}} \hat{Q}_{k}
$$




$$
p_{k}=\left(\frac{1}{\lambda_{k} \hbar^{2}}\right)^{\frac{1}{4}} \hat{P}_{k}
$$

Equation (4) then becomes

$$
\begin{aligned}
H^{o} & =\frac{1}{2} \sum_{k=1}^{3 N-6}\left(\lambda_{k}^{\frac{1}{2}} \hbar\right)\left(p_{k}^{2}+q_{k}^{2}\right) \\
& =\frac{1}{2} h c \sum_{k=1}^{3 N-6} \omega_{k}\left(p_{k}^{2}+q_{k}^{2}\right)
\end{aligned}
$$

The potential $V$ for a polyatomic molecule, which is comprised of the leading harmonic term followed by anharmonic terms, can be expanded as a Taylor series about a minimum energy reference geometry. Two conventions have been used in the literature to denote the potential expansions. One is the restrictive expansion used by Nielsen [1,9-11]

$$
V=\frac{1}{2} h c \sum_{k} \omega_{k} q_{k}^{2}+h c \sum_{k \geq l \geq m} k_{k l m} q_{k} q_{l} q_{m}+h c \sum_{k \geq l \geq m \geq n} k_{k l m n} q_{k} q_{l} q_{m} q_{n}+\ldots
$$

Indices $k, l, m$, and $n$ refer to vibrational modes, $\omega_{k}$ are the harmonic frequencies in wavenumbers, and $k_{k l m}$ and $k_{k l m n}$ are cubic and quartic force constants in wavenumbers. A restrictive summation indicates that only one permutation of the indices $k l m n$ (or $\mathrm{klm}$ ) is included and $k \geq l \geq m \geq n$ in that permutation.

The second convention, used by Mills [12], Aliev and Watson [13], and Papoušek and Aliev [2], includes non-restrictive summations

$$
V=\frac{1}{2} h c \sum_{k} \omega_{k} q_{k}^{2}+h c \sum_{k l m} \frac{1}{3 !} \phi_{k l m} q_{k} q_{l} q_{m}+h c \sum_{k l m n} \frac{1}{4 !} \phi_{k l m n} q_{k} q_{l} q_{m} q_{n}+\ldots
$$

where $\phi_{k l m}$ and $\phi_{k l m n}$ are force constants that are the non-restrictive counterparts of Nielsen's k's. In non-restrictive summations, all permutations of the indices $k l m n$ (or $k l m$ ) are included. Historically $k$ 's were used more frequently in analytical expressions to reduce the number of 
terms written. Recently $\phi$ 's have been used more widely because they are not affected by the number of index permutations and are easier to implement in computational programs.

The $\phi$ 's and $k$ 's can be related by accounting for two constants, the reciprocal

factorials and permutation factors. The reciprocal factorials $\frac{1}{N !}$ in Equation (9) originate from the Taylor expansion of the potential. Permutation factors count the number of possible arrangements of modes. The general form for the permutation factor of a quartic force constant is

$$
\frac{4 !}{N_{k} ! N_{l} ! N_{m} ! N_{n} !}
$$

where $N_{i}$ represents the number of times the index $i$ appears in the force constant. Similarly, the general form for the permutation factor of a cubic force constant is

$$
\frac{3 !}{N_{k} ! N_{l} ! N_{m} !}
$$

Below are some examples of how reciprocal factorials and permutation factors relate non-restrictive and restrictive force constants.

For cases with non-duplicative indices, the permutation factor and reciprocal factorial combine to relate the restrictive and non-restrictive force constants

$$
k_{k l m n}=\frac{4 !}{1 ! 1 ! 1 ! 1 !} \cdot \frac{1}{4 !} \phi_{k l m n}=\phi_{k l m n}
$$

The permutation factor is $\frac{4 !}{1 ! 1 ! 1 ! 1 !}=4$ ! because there are 4 ! ways to arrange four letters where order matters.

For a more specific case, such as $k_{k k l l}$, the restrictive and non-restrictive force constants are not equal 


$$
k_{k k l l}=\frac{4 !}{2 ! 2 !} \cdot \frac{1}{4 !} \phi_{k k l l}=\frac{1}{4} \phi_{k k l l}
$$

Analogous expressions can be evaluated for $k_{k k l m}, k_{k k k l}$, and $k_{k k k k}[14]$

$$
\begin{aligned}
& k_{k k l m}=\frac{1}{2} \phi_{k k l m} \\
& k_{k k k l}=\frac{1}{6} \phi_{k k k l} \\
& k_{k k k k}=\frac{1}{24} \phi_{k k k k}
\end{aligned}
$$

Similarly, the cubic relation involving non-duplicative indices is

$$
k_{k l m}=\frac{3 !}{1 ! 1 ! !} \cdot \frac{1}{3 !} \phi_{k l m}=\phi_{k l m}
$$

For $k_{k l l}$ and $k_{k k k}$,

$$
k_{k l l}=\frac{3 !}{1 ! 2 !} \cdot \frac{1}{3 !} \phi_{k l l}=\frac{1}{2} \phi_{k l l}
$$

and

$$
k_{k k k}=\frac{1}{6} \phi_{k k k}
$$

In this article non-restrictive summations will be used since they yield more manageable expressions and are easier to implement in computational programs. Similar to the above discussion of force constants, both a reciprocal factorial and a permutation factor will be included in non-restrictive summations to equate them to their restrictive counterparts. Key equations are presented in restrictive form in the supplemental material.

By comparing Equations (7) and (9), it is clear that the potential function consists of a leading harmonic term with anharmonic corrections. This form justifies the use of harmonic oscillator basis functions to evaluate matrix elements of the Hamiltonian. The advantage of using these basis functions is that they follow well-known, strict selection rules [1-2]. The 
terms of Equation (7) are purely diagonal, and the additional anharmonic potential terms of Equation (9) result in off-diagonal matrix elements.

The approach taken in this article involves classifying the off-diagonal matrix elements according to the strength of the interactions between states. Small interactions result from nondegenerate anharmonicities and will be treated by second order perturbation theory. Large anharmonic interactions between nearly degenerate basis states arise from resonances and will be treated explicitly. This method of accounting for both large and small interactions among vibrational states is called VPT2+K, which stands for second order vibrational perturbation theory plus resonances. A mathematical process for evaluating VPT2+K matrix elements for nearly degenerate basis states is Van Vleck perturbation theory.

\section{Van Vleck perturbation theory}

The Van Vleck transformation is an approximate method for block-diagonalizing a Hamiltonian matrix [15-20]. In essence, it is perturbation theory applied to not only a single diagonal matrix element, but rather to an entire block of matrix elements.

Consider a Hamiltonian matrix divided into four blocks

$$
H=\left[\frac{H_{2}}{H_{2 \sim 1}} \mid \frac{H_{1 \sim 2}}{H_{1}}\right]
$$

$H_{1}$ and $H_{2}$ are blocks of states where the numbers 1 and 2 denote two different classes of states, class 1 and class 2. Class 1 elements are the strongly interacting states of interest, i.e., states in resonance, and class 2 elements are the remaining states, which might be infinite in number. $H_{1 \sim 2}$ and $H_{2 \sim 1}$ are blocks of interactions between states.

The Van Vleck transformation matrix, represented by the unitary matrix $T$, will be applied to the Hamiltonian to yield the effective Hamiltonian $\widetilde{H}$

$$
\widetilde{H}=T H T^{-1}
$$


with the goal of making $\tilde{H}$ have a form that is nearly

$$
\widetilde{H}=\left[\frac{\tilde{H}_{2}}{0} \mid \frac{0}{\widetilde{H}_{1}}\right]
$$

while not affecting the interactions within the class 1 and class 2 blocks.

$T$ has the effect of folding the class 1-class 2 interactions into the class 1 states of interest while not affecting the class 1 interactions. The matrix form in Equation (20) is approximate because in most cases there will be higher-order off-diagonal terms that appear in higher orders in the zero blocks.

Before defining the transformation, the terms in the original Hamiltonian matrix are expanded by order of importance

$$
H=H^{o}+\lambda H^{\prime}+\lambda^{2} H^{\prime \prime}+\ldots
$$

where $H^{o}$ is the unperturbed system, the primed terms are the perturbations, and $\lambda$ is an ordering parameter. The Hamiltonian has not been expanded past second order here, though in principle it can be expanded to higher orders [7,21]. The transformation will yield an effective Hamiltonian of the form

$$
\widetilde{H}=\widetilde{H}^{o}+\lambda \widetilde{H}^{\prime}+\lambda^{2} \widetilde{H}^{\prime \prime}+\ldots
$$

The Van Vleck transformation matrix $T$ is unitary, meaning that its inverse is equal to its conjugate transpose. Unitary operators can be written as $e^{i M}$, where $M$ is a Hermitian matrix [22]. Thus, $T$ can be expanded as

$$
T=e^{i \lambda S}=1+i \lambda S-\frac{\lambda^{2}}{2} S^{2}+\ldots
$$

where $S$ is a Hermitian matrix. The transformation is applied to the Hamiltonian as in Equation (19) 


$$
\begin{aligned}
\widetilde{H} & =T H T^{-1} \\
& =\left(1+i \lambda S-\frac{\lambda^{2}}{2} S^{2}\right)\left(H^{o}+\lambda H^{\prime}+\lambda^{2} H^{\prime \prime}\right)\left(1-i \lambda S-\frac{\lambda^{2}}{2} S^{2}\right) \\
& =H^{o}-i \lambda H^{o} S+i \lambda S H^{o}+\lambda H^{\prime}-\frac{\lambda^{2}}{2} H^{o} S^{2}+\lambda^{2} S H^{o} S-\frac{\lambda^{2}}{2} S^{2} H^{o}-i \lambda^{2} H^{\prime} S+i \lambda^{2} S H^{\prime} \\
& +\lambda^{2} H^{\prime \prime}-i \frac{\lambda^{3}}{2} S H^{o} S^{2}+i \frac{\lambda^{3}}{2} S^{2} H^{o} S-\frac{\lambda^{3}}{2} H^{\prime} S^{2}+\lambda^{3} S H^{\prime} S-\frac{\lambda^{3}}{2} S^{2} H^{\prime}-i \lambda^{3} H^{\prime \prime} S+i \lambda^{3} S H^{\prime \prime} \\
& +\frac{\lambda^{4}}{2} S^{2} H^{o} S^{2}-i \frac{\lambda^{4}}{2} S H^{\prime} S^{2}+i \frac{\lambda^{4}}{2} S^{2} H^{\prime} S-\frac{\lambda^{4}}{2} H^{\prime \prime} S^{2}+\lambda^{4} S H^{\prime \prime} S-\frac{\lambda^{4}}{2} S^{2} H^{\prime \prime} S \\
& +\frac{\lambda^{5}}{4} S^{2} H^{\prime} S^{2}-\frac{i \lambda^{5}}{2} S H^{\prime \prime} S^{2}+i \frac{\lambda^{5}}{2} S^{2} H^{\prime \prime} S+\frac{\lambda^{6}}{4} S^{2} H^{\prime \prime} S^{2}
\end{aligned}
$$

Since only terms through second order will be utilized, any terms with $\lambda$ degree three or higher has been neglected. Equating the powers of $\lambda$ in Equation (21) and the terms of Equation (24) yields

$$
\begin{aligned}
& \lambda^{o}: \widetilde{H}^{o}=H^{o} \\
& \lambda^{1}: \widetilde{H}^{\prime}=H^{\prime}+i\left(S H^{o}-H^{o} S\right) \\
& \lambda^{2}: \widetilde{H}^{\prime \prime}=H^{\prime \prime}+S H^{o} S-\frac{1}{2}\left(H^{o} S^{2}+S^{2} H^{o}\right)+i\left(S H^{\prime}-H^{\prime} S\right)
\end{aligned}
$$

Two features are essential in the design of the Van Vleck transformation: the transformation cannot alter the interactions between states within a block, and it must yield an approximately block-diagonal matrix, i.e., it must set the class 1 -class 2 matrix elements to zero.

The effective Hamiltonian defined by Equations (25)-(27) will be described in further detail below. Since the class 1 states are the states of interest, their contributions to $\widetilde{H}$ will be emphasized.

The zeroth order term in the effective Hamiltonian, $\tilde{H}^{o}$, simply is $H^{o}$. That is,

$$
\tilde{H}_{a b}^{o}=H_{a b}^{o}
$$


The basis functions are chosen so that $H^{o}$ is diagonal and therefore $H_{a a}^{o}=E_{a}^{o}$, where $a$ represents a class 1 basis function, and $\widetilde{H}_{a b}^{o}=H_{a b}^{o}=0$ if $a \neq b$. Also, the basis functions are selected so that $H_{\gamma \gamma}^{o}=E_{\gamma}^{o}$, where $\gamma$ represents a class 2 basis function. Furthermore, $\tilde{H}_{a \gamma}^{o}=H_{a \gamma}^{o}=0$. In summary,

$$
\tilde{H}_{i j}^{o}=E_{i}^{o} \delta_{i j}
$$

where $i$ and $j$ could be either class 1 or class 2 states.

The Van Vleck transformation does not affect the class 1-class 1 or class 2-class 2 interactions. In order for this to occur, the following conditions must be met

$$
S_{a b}=0
$$

and

$$
S_{\gamma \beta}=0
$$

where $b$ is a class 1 basis function and $\beta$ is a class 2 basis function. The condition in Equation (30) prevents the transformation from acting on the interactions between class 1 states.

Similarly, the condition in Equation (31) insures that the transformation does not affect the interactions between class 2 states.

Additionally, the class 1-class 2 (and class 2-class 1) matrix elements of $\widetilde{H}$ must be zero to second order so that the effective Hamiltonian is approximately block-diagonal. To insure that $\tilde{H}_{a \gamma}^{\prime}=0$, the following condition must be met

$$
S_{a \gamma}=\frac{-i H_{a \gamma}^{\prime}}{E_{a}^{o}-E_{\gamma}^{o}}
$$

Note that defining the Van Vleck transformation as $\widetilde{H}=T^{-1} H T$ will introduce a sign difference $[18,20]$ in Equation (32), but the final result will be the same.

Using Equations (30)-(32), the class 1-class 2 interactions in Equation (26) become 


$$
\begin{aligned}
\tilde{H}_{a \gamma}^{\prime} & =H_{a \gamma}^{\prime}+i\left[\left(S H^{o}\right)_{a \gamma}-\left(H^{o} S\right)_{a \gamma}\right] \\
& =H_{a \gamma}^{\prime}+i\left(S_{a \gamma} H_{\gamma \gamma}^{o}-H_{a a}^{o} S_{a \gamma}\right) \\
& =H_{a \gamma}^{\prime}+i \frac{(-i) H_{a \gamma}^{\prime}}{E_{a}^{o}-E_{\gamma}^{o}} E_{\gamma}^{o}-i E_{a}^{o} \frac{(-i) H_{a \gamma}^{\prime}}{E_{a}^{o}-E_{\alpha}^{o}} \\
& =H_{a \gamma}^{\prime}+\frac{H_{a \gamma}^{\prime} E_{\gamma}^{o}}{E_{a}^{o}-E_{\gamma}^{o}}-\frac{E_{a}^{o} H_{a \gamma}^{\prime}}{E_{a}^{o}-E_{\gamma}^{o}} \\
& =0
\end{aligned}
$$

demonstrating that Equation (32) block-diagonalizes $\widetilde{H}^{\prime}$ to second order. By the definition of matrix multiplication, $\left(S H^{o}\right)_{a \gamma}=\sum_{i} S_{a i} H_{i \gamma}^{o}$ and $\left(H^{o} S\right)_{a \gamma}=\sum_{i} H_{a i}^{o} S_{i \gamma}$ where $i$ represents a state through which class 1 state $a$ and class 2 state $\gamma$ interact. Since $S_{a b}=0$ and $S_{\gamma \beta}=0$, only $S_{a \gamma}$ is nonzero. Since $H^{o}$ is diagonal, only $H^{o}{ }_{a a}$ and $H^{o}{ }_{r y}$ are nonzero. Then the nonzero matrix elements $\left(S H^{o}\right)_{a \gamma}$ and $\left(H^{o} S\right)_{a \gamma}$ are $S_{a \gamma} H_{\gamma \gamma}^{o}$ and $H_{a a}^{o} S_{a \gamma}$, respectively.

As a result of Equation (33), the effect of the class 1-class 2 matrix elements is incorporated into the class 1 block of the Hamiltonian [18]. In using Equation (26), three types of matrix elements must be considered: class 1-class 1, class 1-class 2, and class 2-class 2. Setting the class 1-class 2 matrix elements $\widetilde{H}_{a \gamma}^{\prime}$ equal to zero, $H_{a \gamma}^{\prime}=i\left\lfloor\left(H^{o} S\right)_{a \gamma}-\left(S H^{o}\right)_{a \gamma}\right\rfloor$. Thus, the class 1-class 2 elements are exactly cancelled out in $H^{\prime}$ by the transformation, the only nonzero parts of $H^{\prime}$ arise from $H_{a b}^{\prime}$ and $H_{\gamma \beta}^{\prime}$, and the first order energy correction to the class 1 states, $\widetilde{H}_{a b}^{\prime}$, is $H_{a b}^{\prime}$

$$
\widetilde{H}_{a b}^{\prime}=H_{a b}^{\prime}
$$

The second order energy correction term to class 1 states, $\widetilde{H}_{a b}^{\prime \prime}$, is formulated below. To ease the derivation, Equation (27) is rearranged 


$$
\begin{aligned}
\widetilde{H}^{\prime \prime} & =H^{\prime \prime}+i\left(S H^{\prime}-H^{\prime} S\right)+S H^{o} S-\frac{1}{2}\left(S^{2} H^{o}+H^{o} S^{2}\right) \\
& =H^{\prime \prime}+i\left(S H^{\prime}-H^{\prime} S\right)-\frac{1}{2}\left(S^{2} H^{o}-2 S H^{o} S+H^{o} S^{2}\right) \\
& =H^{\prime \prime}+i S H^{\prime}-i H^{\prime} S+\frac{1}{2}\left[S\left(H^{o} S-S H^{o}\right)-\left(H^{o} S-S H^{o}\right) S\right] \\
& =H^{\prime \prime}+i S H^{\prime}-i H^{\prime} S+\frac{i}{2}\left[-i S\left(H^{o} S-S H^{o}\right)+\left(H^{o} S-S H^{o}\right) i S\right] \\
& =H^{\prime \prime}+\frac{i}{2}\left[2 S H^{\prime}-2 H^{\prime} S+i S\left(S H^{o}-H^{o} S\right)-\left(S H^{o}-H^{o} S\right) i S\right] \\
& =H^{\prime \prime}+\frac{i}{2}\left\{S\left[2 H^{\prime}+i\left(S H^{o}-H^{o} S\right)\right]-\left[2 H^{\prime}+\left(S H^{o}-H^{o} S\right) i\right] S\right\} \\
& =H^{\prime \prime}+\frac{i}{2}\left[S\left(H^{\prime}+\tilde{H}^{\prime}\right)-\left(H^{\prime}+\tilde{H}^{\prime}\right) S\right] \\
& =H^{\prime \prime}+\frac{i}{2}\left[\left(S H^{\prime}-H^{\prime} S\right)+\left(S \tilde{H}^{\prime}-\tilde{H}^{\prime} S\right)\right]
\end{aligned}
$$

where Equation (26) has been used to simplify the result.

$\widetilde{H}_{a b}^{\prime \prime}$ is obtained by utilizing the final expression in Equation (35)

$$
\begin{aligned}
\tilde{H}_{a b}^{\prime \prime} & =H_{a b}^{\prime \prime}+\frac{i}{2}\left[\left(S H^{\prime}-H^{\prime} S\right)_{a b}+\left(S \tilde{H}^{\prime}-\tilde{H}^{\prime} S\right)_{a b}\right] \\
& =H_{a b}^{\prime \prime}+\frac{i}{2}\left[\left(S H^{\prime}\right)_{a b}-\left(H^{\prime} S\right)_{a b}+\left(S \tilde{H}^{\prime}\right)_{a b}-\left(\tilde{H}^{\prime} S\right)_{a b}\right]
\end{aligned}
$$

As mentioned previously, by the definition of matrix multiplication, $\left(S H^{\prime}\right)_{a b}=\sum_{i} S_{a i} H_{i b}^{\prime}$ where $i$ represents a state through which class 1 states $a$ and $b$ interact. Since $S_{a b}=0$ and $S_{\gamma \beta}=0$, only $S_{a \gamma}$ and $S_{\gamma a}$ are nonzero. This implies that class 1 states $a$ and $b$ can only interact with each other via a class 2 state $\gamma$

$$
\tilde{H}_{a b}^{\prime \prime}=H_{a b}^{\prime \prime}+\frac{i}{2} \sum_{\gamma}\left(S_{a \gamma} H_{\gamma b}^{\prime}-H_{a \gamma}^{\prime} S_{\gamma b}+S_{a \gamma} \tilde{H}_{\gamma b}^{\prime}-\tilde{H}_{a \gamma}^{\prime} S_{\gamma b}\right)
$$

Recalling that $\tilde{H}_{b \gamma}^{\prime}=0$ and $\tilde{H}_{\gamma b}^{\prime}=0$ and the definition of $S_{a \gamma}$ from Equation (32) 


$$
\begin{aligned}
\widetilde{H}_{a b}^{\prime \prime} & =H_{a b}^{\prime \prime}+\frac{i}{2} \sum_{\gamma}\left(S_{a \gamma} H_{\gamma b}^{\prime}-H_{a \gamma}^{\prime} S_{\gamma b}\right) \\
& =H_{a b}^{\prime \prime}+\frac{i}{2} \sum_{\gamma}\left(\frac{-i H_{a \gamma}^{\prime} H_{\gamma b}^{\prime}}{E_{a}^{o}-E_{\gamma}^{o}}-\frac{H_{a \gamma}^{\prime}(-i) H_{\gamma b}^{\prime}}{E_{\gamma}^{o}-E_{b}^{o}}\right) \\
& =H_{a b}^{\prime \prime}+\frac{1}{2} \sum_{\gamma}\left(\frac{H_{a \gamma}^{\prime} H_{\gamma b}^{\prime}}{E_{a}^{o}-E_{\gamma}^{o}}-\frac{H_{a \gamma}^{\prime} H_{\gamma b}^{\prime}}{E_{\gamma}^{o}-E_{b}^{o}}\right) \\
& =H_{a b}^{\prime \prime}+\frac{1}{2} \sum_{\gamma}\left(\frac{H_{a \gamma}^{\prime} H_{\gamma b}^{\prime}}{E_{a}^{o}-E_{\gamma}^{o}}+\frac{H_{a \gamma}^{\prime} H_{\gamma b}^{\prime}}{E_{b}^{o}-E_{\gamma}^{o}}\right)
\end{aligned}
$$

Combining the final expressions for the zeroth, first, and second order energy corrections given in Equations (28), (34), and (38), $\widetilde{H}_{a b}$ becomes

$$
\tilde{H}_{a b}=\tilde{H}_{a b}^{o}+\lambda \widetilde{H}_{a b}^{\prime}+\lambda^{2} \widetilde{H}_{a b}^{\prime \prime}=H^{o}+\lambda H_{a b}^{\prime}+\lambda^{2} H_{a b}^{\prime \prime}+\frac{\lambda^{2}}{2} \sum_{\gamma}\left(\frac{H_{a \gamma}^{\prime} H_{\gamma b}^{\prime}}{E_{a}^{o}-E_{\gamma}^{o}}+\frac{H_{a \gamma}^{\prime} H_{\gamma b}^{\prime}}{E_{b}^{o}-E_{\gamma}^{o}}\right)
$$

Equation (39) is the same as Lefebvre and Field's Equation (4.2.17) but with the addition of $\lambda^{2} H_{a b}^{\prime \prime}$ because the second order term in Equation (21) is included here [18].

Since $\lambda$ is an arbitrary ordering parameter, it can be set to 1 , yielding the final result

$$
\widetilde{H}_{a b}=\widetilde{H}_{a b}^{o}+\widetilde{H}_{a b}^{\prime}+\widetilde{H}_{a b}^{\prime \prime}=H_{a b}^{o}+H_{a b}^{\prime}+H_{a b}^{\prime \prime}+\frac{1}{2} \sum_{\gamma}\left(\frac{H_{a \gamma}^{\prime} H_{\gamma b}^{\prime}}{E_{a}^{o}-E_{\gamma}^{o}}+\frac{H_{a \gamma}^{\prime} H_{\gamma b}^{\prime}}{E_{b}^{o}-E_{\gamma}^{o}}\right)
$$

where it should be emphasized that the sum over $\gamma$ is a sum over all class 2 states but not over class 1 states.

Equation (40) forms the foundation of the VPT2+K approach because it simultaneously treats a block of both diagonal and off-diagonal strongly interacting class 1 states along with weaker class 1-class 2 (second-order) interactions. Van Vleck perturbation theory is more general than nondegenerate second-order perturbation theory and in fact contains it as a special case in the diagonal matrix elements. To see this, letting $b=a$ in the second order energy correction of Equation (40) yields 


$$
\begin{aligned}
\tilde{H}_{a a}^{\prime \prime} & =H_{a a}^{\prime \prime}+\frac{1}{2} \sum_{\gamma}\left(\frac{H_{a \gamma}^{\prime} H_{\gamma a}^{\prime}}{E_{a}^{o}-E_{\gamma}^{o}}+\frac{H_{a \gamma}^{\prime} H_{\gamma a}^{\prime}}{E_{a}^{o}-E_{\gamma}^{o}}\right) \\
& =H_{a a}^{\prime \prime}+\sum_{\gamma} \frac{H_{a \gamma}^{\prime} H_{\gamma a}^{\prime}}{E_{a}^{o}-E_{\gamma}^{o}}
\end{aligned}
$$

which is identical to the diagonal second-order matrix element derived via nondegenerate second-order perturbation theory $[1,17]$.

The advantage of Van Vleck perturbation theory over nondegenerate second-order perturbation theory is that both on- and off-diagonal matrix elements of class 1 states can be found using Van Vleck perturbation theory, whereas only diagonal elements result from nondegenerate second-order perturbation theory. Van Vleck perturbation theory allows strongly interacting states to be treated as class 1 states and all other weakly interacting states as class 2 states.

In principle, the Van Vleck transformation described here and contact transformations used by Nielsen [9-11] and by Papoušek and Aliev [2] are the same, but the application of the transformations differs. Those who utilize Van Vleck perturbation theory tend to focus on evaluating matrix elements, whereas those who use contact transformations tend to focus on deriving $S$ matrices for particular situations. In the following section, Equation (40) will be used to transform the vibrational Hamiltonian of a polyatomic molecule and derive explicit expressions for both on- and off-diagonal matrix elements of a set of strongly interacting states, which in turn weakly interact with the other vibrational states.

\section{Transformation of the Hamiltonian}

\section{A. Diagonal matrix elements}

\section{Identification of transformed vibrational Hamiltonian matrix elements}

Matrix elements of the vibrational Hamiltonian for a polyatomic molecule will now be derived using Van Vleck perturbation theory with harmonic oscillator basis functions. The 
diagonal matrix elements will be evaluated first, since although this process is tedious, it is the most beneficial way to introduce much of the reasoning that will be used for evaluating the off-diagonal matrix elements.

Normal mode harmonic oscillator basis functions are used for the evaluation of matrix elements for three reasons. First, they are diagonal in $H^{o}$, the zeroth-order term in the vibrational Hamiltonian, as required by Van Vleck perturbation theory. Second, they follow strict selection rules shown in Table 1, which are beneficial when summing over an infinite number of states [1-2]. Third, since the coordinates are orthogonal, matrix elements of different modes can be factored from each other, and constants can be factored from the matrix elements

$$
\left\langle\mathrm{v}_{k}, \mathrm{v}_{l}, \mathrm{v}_{m}\left|\phi_{k l m} q_{k} q_{l} q_{m}\right| \mathrm{v}_{k}+1, \mathrm{v}_{l}+1, \mathrm{v}_{m}+1\right\rangle=\phi_{k l m}\left\langle\mathrm{v}_{k}\left|q_{k}\right| \mathrm{v}_{k}+1\right\rangle\left\langle\mathrm{v}_{l}\left|q_{l}\right| \mathrm{v}_{l}+1\right\rangle\left\langle\mathrm{v}_{m}\left|q_{m}\right| \mathrm{v}_{m}+1\right\rangle
$$

which greatly simplifies their evaluation.

The process for identifying the nonzero diagonal matrix elements is motivated by Califano's [1] derivation of the nonzero diagonal matrix elements using nondegenerate second order perturbation theory. However, the derivation in this article uses Van Vleck perturbation theory instead of nondegenerate second order perturbation theory, utilizes nonrestrictive instead of restrictive summations, and includes the vibrational angular momentum term in the vibrational Hamiltonian.

By using non-restrictive summations in all of the expressions, all possible matrix elements will be evaluated, though many will be zero due to the strict selection rules of the harmonic oscillator basis functions.

Recall that the original Hamiltonian can be written as

$$
H=H^{o}+H^{\prime}+H^{\prime \prime}
$$


where the arbitrary ordering parameter $\lambda$ in Equation (21) is set to 1. In the vibrational Hamiltonian, $H^{o}$ is the harmonic oscillator, $H^{\prime}$ contains the cubic potential terms, and $H^{\prime \prime}$ contains the quartic potential and vibrational angular momentum terms

$$
\begin{gathered}
H^{o}=\frac{1}{2} h c \sum_{k} \omega_{k}\left(p_{k}^{2}+q_{k}^{2}\right) \\
H^{\prime}=h c \sum_{k l m} \frac{1}{3 !} \phi_{k l m} q_{k} q_{l} q_{m} \\
H^{\prime \prime}=h c \sum_{k l m n} \frac{1}{4 !} \phi_{k l m n} q_{k} q_{l} q_{m} q_{n}+\frac{1}{2} \sum_{\alpha \beta} \mu_{\alpha \beta} \hat{\pi}_{\alpha} \hat{\pi}_{\beta}
\end{gathered}
$$

After applying the Van Vleck transformation, the effective Hamiltonian

$$
\widetilde{H}=\widetilde{H}^{o}+\widetilde{H}^{\prime}+\widetilde{H}^{\prime \prime}
$$

is obtained, where the arbitrary ordering parameter $\lambda$ in Equation (22) is set to 1 . From Equation (40), the matrix elements of $\widetilde{H}$ can be written as

$$
\begin{aligned}
& \tilde{H}_{a b}^{o}=H_{a b}^{o}=\left\langle a \mid \frac{1}{2} h c \sum_{k} \omega_{k}\left(p_{k}^{2}+q_{k}^{2}\right) b\right\rangle \\
& \tilde{H}_{a b}^{\prime}=H_{a b}^{\prime}=\left\langle a\left|h c \sum_{k l m} \frac{1}{3 !} \phi_{k l m} q_{k} q_{l} q_{m}\right| b\right\rangle \\
& \tilde{H}_{a b}^{\prime \prime}=H_{a b}^{\prime \prime}+\frac{1}{2} \sum_{\gamma}\left(\frac{H_{a \gamma}^{\prime} H_{\gamma b}^{\prime}}{E_{a}^{o}-E_{\gamma}^{o}}+\frac{H_{a \gamma}^{\prime} H_{\gamma b}^{\prime}}{E_{b}^{o}-E_{\gamma}^{o}}\right) \\
& =\left\langle a\left|h c \sum_{k l m n} \frac{1}{4 !} \phi_{k l m n} q_{k} q_{l} q_{m} q_{n}\right| b\right\rangle+\left\langle a\left|\frac{1}{2} \sum_{\alpha \beta} \mu_{\alpha \beta} \hat{\pi}_{\alpha} \hat{\pi}_{\beta}\right| b\right\rangle \\
& +\frac{1}{2} h^{2} c^{2} \sum_{\gamma \neq a, b}\left(\frac{\left\langle a\left|\sum_{k l m} \frac{1}{3 !} \phi_{k l m} q_{k} q_{l} q_{m}\right| \gamma\right\rangle\left\langle\gamma\left|\sum_{k^{\prime} l^{\prime} m^{3}} \frac{1}{3 !} \phi_{k^{\prime} l^{\prime} m^{\prime}} q_{k^{\prime}} q_{l^{\prime}} q_{m^{\prime}}\right| b\right\rangle}{E_{a}^{o}-E_{\gamma}^{o}}\right. \\
& \left.+\frac{\left\langle a\left|\sum_{k l m} \frac{1}{3 !} \phi_{k l m} q_{k} q_{l} q_{m}\right| \gamma\right\rangle\left\langle\gamma\left|\sum_{k^{\prime} l^{\prime} m^{3}} \frac{1}{3 !} \phi_{k^{\prime} l^{\prime} m^{\prime}} q_{k^{\prime}} q_{l^{\prime}} q_{m^{\prime}}\right| b\right\rangle}{E_{b}^{o}-E_{\gamma}^{o}}\right)
\end{aligned}
$$




\section{Zeroth order terms}

The nonzero matrix elements of the zeroth order term in the effective Hamiltonian

will be evaluated. As seen in Equation (47), the zeroth order transformed Hamiltonian $\widetilde{H}^{o}$ is the harmonic oscillator Hamiltonian $H^{o}$. The matrix elements of the harmonic oscillator are nonzero only when $a=b$ because the zeroth order Hamiltonian is diagonal in class 1 states $a$. The diagonal matrix elements of (47) have the general form

$$
h c\left\langle a\left|\frac{1}{2} \sum_{k} \omega_{k}\left(p_{k}^{2}+q_{k}^{2}\right)\right| a\right\rangle
$$

Using Table 1, the one-dimensional nonzero matrix element is

$$
\left\langle\mathrm{v}_{k}\left|\omega_{k}\left(p_{k}^{2}+q_{k}^{2}\right)\right| \mathrm{v}_{k}\right\rangle=2 \omega_{k}\left(\mathrm{v}_{k}+\frac{1}{2}\right)
$$

Thus, the diagonal zeroth order matrix elements due to the potential of the harmonic oscillator, when summed over all possible modes $k$, have the form

$$
\left\langle a\left|\tilde{H}^{o}\right| a\right\rangle=h c\left\langle a\left|\frac{1}{2} \sum_{k} \omega_{k}\left(p_{k}^{2}+q_{k}^{2}\right)\right| a\right\rangle=h c \sum_{k} \omega_{k}\left(\mathrm{v}_{k}+\frac{1}{2}\right)
$$

Equation (51) represents the total zeroth-order energy contribution, and

$$
\left\langle a\left|\tilde{H}^{o}\right| a\right\rangle=\left\langle a\left|H^{o}\right| a\right\rangle
$$

and

$$
\tilde{H}_{a a}^{o}=H_{a a}^{o}
$$

\section{First order terms}

As seen in Equation (48), the first order energy correction to the potential is $H^{\prime}$, the cubic term. The most general diagonal matrix element is

$$
\tilde{H}_{a a}^{\prime}=H_{a a}^{\prime}=h c\left\langle a\left|\sum_{k l m} \frac{1}{3 !} \phi_{k l m} q_{k} q_{l} q_{m}\right| a\right\rangle
$$


The possible matrix elements have the force constants $\phi_{k l m}, \phi_{k k l}$, and $\phi_{k k k}$, where $k, l \neq m$. The diagonal matrix elements involving these constants are always zero because they will always include an odd power of $q_{i}$, and $\left\langle\mathrm{v}_{i}\left|q_{i}\right| \mathrm{v}_{i}\right\rangle=0$ and $\left\langle\mathrm{v}_{i}\left|q_{i}^{3}\right| \mathrm{v}_{i}\right\rangle=0$. In general, for diagonal matrix elements, if the total degree of some $q_{i}$ is odd, then the entire matrix element will be zero.

Since all diagonal cubic matrix elements will have at least one $q_{i}$ of odd degree, all the diagonal matrix elements of the first order correction are zero

$$
\left\langle a\left|\tilde{H}_{a a}^{\prime}\right| a\right\rangle=0
$$

and

$$
\tilde{H}_{a a}^{\prime}=0
$$

\section{Second order terms}

According to Equation (49), the diagonal matrix element for the second order energy correction to the potential is

$$
\begin{aligned}
\tilde{H}_{a a}^{\prime \prime} & =H_{a a}^{\prime \prime}+\sum_{\gamma \neq a} \frac{H_{a \gamma}^{\prime} H_{\gamma a}^{\prime}}{E_{a}^{o}-E_{\gamma}^{o}} \\
& =h c\left\langle a\left|\sum_{k l m n} \frac{1}{4 !} \phi_{k l m n} q_{k} q_{l} q_{m} q_{n}\right| a\right\rangle+h^{2} c^{2} \sum_{\gamma \neq a} \frac{\left\langle a\left|\sum_{k l m} \frac{1}{3 !} \phi_{k l m} q_{k} q_{l} q_{m}\right| \gamma\right\rangle\left\langle\gamma\left|\sum_{k^{\prime} l^{\prime} m^{3}} \frac{1}{3 !} \phi_{k^{\prime} l^{\prime} m^{\prime}} q_{k^{\prime}} q_{l^{\prime}} q_{m^{\prime}}\right| a\right\rangle}{E_{a}^{o}-E_{\gamma}^{o}}
\end{aligned}
$$

Note that this expression is only the correction to the potential. It does not include the vibrational angular momentum term, which will be treated in the next subsection. In the second term of Equation (57), the sum over $\gamma$ is a sum over all class 2 states. 
The first term of Equation (57) will be evaluated. There are five possible types of matrix elements with the following permutation factors, reciprocal factorials, and force constants

$$
\frac{4 !}{1 ! 1 ! 1 ! !} \cdot \frac{1}{4 !} \phi_{k l m n} \quad \frac{4 !}{2 ! 1 ! 1 !} \cdot \frac{1}{4 !} \phi_{k k l m} \quad \frac{4 !}{2 ! 2 !} \cdot \frac{1}{4 !} \phi_{k k l l} \quad \frac{4 !}{3 ! 1 !} \cdot \frac{1}{4 !} \phi_{k k k l} \quad \frac{4 !}{4 !} \cdot \frac{1}{4 !} \phi_{k k k k}
$$

where $k \neq l \neq m \neq n$. The permutation factors are included because the summations in Equation (57) are non-restrictive, and the reciprocal factorials originate in the Taylor expansion of the potential.

The reasoning applied in the evaluation of the first order energy correction is relevant here: only two of the five possible types of matrix elements are nonzero, and they involve the force constants $\phi_{k k k k}$ and $\phi_{k k l l}$. The other three types are zero because each one has at least one $q_{i}$ of odd degree. Using harmonic oscillator matrix elements from Table 1, the nonzero matrix elements are

$$
\begin{gathered}
\left\langle\mathrm{v}_{k}\left|\phi_{k k k k} q_{k}^{4}\right| \mathrm{v}_{k}\right\rangle=\phi_{k k k k}\left\langle\mathrm{v}_{k}\left|q_{k}^{4}\right| \mathrm{v}_{k}\right\rangle=\frac{3}{2} \phi_{k k k k}\left[\left(\mathrm{v}_{k}+\frac{1}{2}\right)^{2}+\frac{1}{4}\right] \\
\left\langle\mathrm{v}_{k}, \mathrm{v}_{l}\left|\phi_{k k l l} q_{k}^{2} q_{l}^{2}\right| \mathrm{v}_{k}, v_{l}\right\rangle=\phi_{k k l l}\left\langle\mathrm{v}_{k}\left|q_{k}^{2}\right| \mathrm{v}_{k}\right\rangle\left\langle\mathrm{v}_{l}\left|q_{l}^{2}\right| \mathrm{v}_{l}\right\rangle=\phi_{k k l l}\left(\mathrm{v}_{k}+\frac{1}{2}\right)\left(\mathrm{v}_{l}+\frac{1}{2}\right)
\end{gathered}
$$

Then the first term of Equation (57) becomes

$$
\begin{aligned}
h c\left\langle a\left|\sum_{k l m n} \frac{1}{4 !} \phi_{k l m n} q_{k} q_{l} q_{m} q_{n}\right| a\right\rangle & =h c \sum_{k} \frac{4 !}{4 !} \cdot \frac{1}{4 !} \phi_{k k k k} \cdot \frac{3}{2}\left[\left(\mathrm{v}_{k}+\frac{1}{2}\right)^{2}+\frac{1}{4}\right]+h c \frac{1}{2 !} \sum_{k \neq l} \frac{4 !}{2 ! 2 !} \cdot \frac{1}{4 !} \phi_{k k l l}\left(\mathrm{v}_{k}+\frac{1}{2}\right)\left(v_{l}+\frac{1}{2}\right) \\
& =\frac{1}{16} h c \sum_{k} \phi_{k k k k}\left[\left(\mathrm{v}_{k}+\frac{1}{2}\right)^{2}+\frac{1}{4}\right]+\frac{1}{8} h c \sum_{k \neq l} \phi_{k k l l}\left(\mathrm{v}_{k}+\frac{1}{2}\right)\left(\mathrm{v}_{l}+\frac{1}{2}\right)
\end{aligned}
$$


Equation (60) presents the non-restrictive representation of the second order quartic correction. For example, on the left-hand side, the summation includes six force constants equivalent to $\phi_{k k l l}, e . g ., \phi_{k l k l}$ and $\phi_{k l l k}$. The permutation factors of non-restrictive force constants account for all permutations. On the right-hand side, the six permutations of $k k l l$ are incorporated into the $\phi_{k k l l}$ term by the permutation factor $\frac{4 !}{2 ! 2 !}$. However, the non-restrictive force constant and its permutation factor, the non-restrictive sum over $k$ and $l$ regenerates some of the permutations, e.g., $\phi_{1122}$ and $\phi_{2211}$. To weight each permutation as counted only once, a $\frac{1}{2 !}$ factor is introduced for the non-restrictive sum with two indices.

The second term of Equation (57) requires a more involved derivation, which will be presented using non-restrictive summations that will be adjusted so that only one unique term of each type is counted in the summation. It is important to note that each term is a product of two off-diagonal matrix elements that are connected by a common intermediate state.

Moreover, the summations over modes $k, l$, and $m$ are independent of the summations over $k$, $l$ ', and $m^{\prime}$. Consequently, there could be a maximum of six distinct modes involved in this energy correction term.

Similar to the first order term, if the total degree of $q_{i}$ for some $i$ is odd in the second term of Equation (57), the entire matrix element will be zero. For example, although $\left\langle a\left|q_{i}\right| \gamma\right\rangle$ would be nonzero if the quanta in mode $i$ differed by one quantum between states $a$ and $\gamma$, an odd power of $q_{i}, e . g .,\left\langle\gamma\left|q_{i}^{3}\right| a\right\rangle$, would be needed to return to state $a$ in a nonzero matrix element so that the total degree of $q_{i}$ is even. If the total degree of $q_{i}$ of both matrix elements is odd, then it is not possible for state $a$ to connect back to itself.

If there are six distinct modes $\left(k \neq l \neq m \neq k^{\prime} \neq l^{\prime} \neq m^{\prime}\right)$, then each $q_{i}$ will have a degree of one. Consequently, there will be three distinct $q_{i}$ in each bra-ket of the second term of 
Equation (57). This prevents the quanta changed in the intermediate state $\gamma$ to return to their original number in class 1 state $a$. Therefore, there will be no common intermediate state for which both matrix elements are nonzero. Thus, there are no nonzero matrix elements when six distinct modes are considered.

Similarly, if there are five distinct modes $\left(k \neq l \neq m \neq k^{\prime} \neq l^{\prime}\right)$, there can be no common intermediate state with a nonzero matrix element for four of the five modes since one is the degree of $q_{i}$ for four of the five modes. Hence, there are no nonzero terms when five distinct modes are considered.

Since any odd power of $q_{i}$ will not lead to any nonzero matrix elements in the second term of Equation (57), when four distinct modes $\left(k \neq l \neq m \neq k^{\prime}\right)$ are considered, the matrix element is zero. Two cases are possible: either two modes' $q_{i}$ will have degree one overall and two modes' $q_{i}$ will have degree two, or three modes will have $q_{i}$ of degree one and one mode will have $q_{i}$ of degree three overall. In either case, since there are modes with $q_{i}$ of degree one, state $a$ cannot be reconnected with itself via state $\gamma$, and the overall matrix element is zero. Thus, there are no nonzero terms when six, five, or four distinct modes are involved. However, there can be nonzero terms when three, two, or one distinct modes are considered. These terms will be formulated below.

For matrix elements with three distinct modes $(k \neq l \neq m)$, seven nonzero terms with the following potential constants, permutation factors, and reciprocal factorials are possible:

$$
\begin{array}{ccc}
\frac{3 !}{1 ! 1 ! !} \cdot \frac{1}{3 !} \cdot \phi_{k l m} \frac{3 !}{1 ! 1 ! ! !} \cdot \frac{1}{3 !} \phi_{k l m} & \frac{3 !}{2 ! !} \cdot \frac{1}{3 !} \cdot \phi_{k k l} \frac{3 !}{2 ! 1 !} \cdot \frac{1}{3 !} \phi_{l m m} & \frac{3 !}{2 ! 1 !} \cdot \frac{1}{3 !} \cdot \phi_{l m m} \frac{3 !}{2 ! 1 !} \cdot \frac{1}{3 !} \phi_{k k l} \\
\frac{3 !}{2 ! ! !} \cdot \frac{1}{3 !} \cdot \phi_{k k m} \frac{3 !}{2 ! !} \cdot \frac{1}{3 !} \phi_{l l m} & \frac{3 !}{2 ! ! !} \cdot \frac{1}{3 !} \cdot \phi_{l l m} \frac{3 !}{2 ! ! !} \cdot \frac{1}{3 !} \phi_{k k m} & \frac{3 !}{2 ! !} \cdot \frac{1}{3 !} \cdot \phi_{k l l} \frac{3 !}{2 ! ! !} \cdot \frac{1}{3 !} \phi_{k m m} \\
\frac{3 !}{2 ! !} \cdot \frac{1}{3 !} \cdot \phi_{k m m} \frac{3 !}{2 ! !} \cdot \frac{1}{3 !} \phi_{k l l}
\end{array}
$$


Other matrix elements with three distinct modes are possible, but they do not lead to nonzero matrix elements due to an odd degree in some $q_{i}$. Since the last six terms are of the same type, they will eventually be collapsed into one summation. It is important to note that matrix element types with the order of the force constants switched (e.g., $\phi_{k k l} \phi_{l m m}$ and $\left.\phi_{l m m} \phi_{k k l}\right)$ are mathematically equivalent:

$$
\begin{aligned}
& \left\langle\mathrm{v}_{k}, \mathrm{v}_{l}, \mathrm{v}_{m}\left|\phi_{k k l} q_{k}^{2} q_{l}\right| \mathrm{v}_{k}, \mathrm{v}_{l}+1, \mathrm{v}_{m}\right\rangle\left\langle\mathrm{v}_{k}, \mathrm{v}_{l}+1, \mathrm{v}_{m}\left|\phi_{l m m} q_{l} q_{m}^{2}\right| \mathrm{v}_{k}, \mathrm{v}_{l}, \mathrm{v}_{m}\right\rangle \\
& =\phi_{k k l} \phi_{l m m}\left\langle\mathrm{v}_{k}\left|q_{k}^{2}\right| \mathrm{v}_{k}\right\rangle\left\langle\mathrm{v}_{l}\left|q_{l}\right| \mathrm{v}_{l}+1\right\rangle\left\langle\mathrm{v}_{m} \mid \mathrm{v}_{m}\right\rangle\left\langle\mathrm{v}_{k} \mid \mathrm{v}_{k}\right\rangle\left\langle\mathrm{v}_{l}+1\left|q_{l}\right| \mathrm{v}_{l}\right\rangle\left\langle\mathrm{v}_{m}\left|q_{l}^{2}\right| \mathrm{v}_{m}\right\rangle \\
& =\phi_{l m m} \phi_{k k l}\left\langle\mathrm{v}_{k} \mid \mathrm{v}_{k}\right\rangle\left\langle\mathrm{v}_{l}\left|q_{l}\right| \mathrm{v}_{l}+1\right\rangle\left\langle\mathrm{v}_{m}\left|q_{l}^{2}\right| \mathrm{v}_{m}\right\rangle\left\langle\mathrm{v}_{k}\left|q_{k}^{2}\right| \mathrm{v}_{k}\right\rangle\left\langle\mathrm{v}_{l}+1\left|q_{l}\right| \mathrm{v}_{l}\right\rangle\left\langle\mathrm{v}_{m} \mid \mathrm{v}_{m}\right\rangle \\
& =\left\langle\mathrm{v}_{k}, \mathrm{v}_{l}, \mathrm{v}_{m}\left|\phi_{l m m} q_{l} q_{m}^{2}\right| \mathrm{v}_{k}, \mathrm{v}_{l}+1, \mathrm{v}_{m}\right\rangle\left\langle\mathrm{v}_{k}, \mathrm{v}_{l}+1, \mathrm{v}_{m}\left|\phi_{k k l} q_{k}^{2} q_{l}\right| \mathrm{v}_{k}, \mathrm{v}_{l}, \mathrm{v}_{m}\right\rangle
\end{aligned}
$$

Since the two are mathematically equivalent, these terms will be combined, and a factor of two will be included in the relevant subsequent expression.

For matrix elements with two distinct modes $(k \neq l)$, three nonzero terms with the following force constants, permutation factors, and reciprocal factorials are possible:

$$
\frac{3 !}{3 !} \cdot \frac{1}{3 !} \cdot \phi_{k k k} \frac{3 !}{1 ! 2 !} \cdot \frac{1}{3 !} \phi_{k l l} \quad \frac{3 !}{1 ! 2 !} \cdot \frac{1}{3 !} \phi_{k l l} \frac{3 !}{3 !} \cdot \frac{1}{3 !} \cdot \phi_{k k k} \quad \frac{3 !}{2 ! 1 !} \cdot \frac{1}{3 !} \cdot \phi_{k k l} \frac{3 !}{2 ! 1 !} \cdot \frac{1}{3 !} \phi_{k k l}
$$

Of these, the matrix elements with the first two sets of constants will be collapsed into one summation.

For matrix elements with one distinct mode, only one matrix element type with the following force constants, permutation factors, and reciprocal factorials is possible:

$$
\frac{3 !}{3 !} \cdot \frac{1}{3 !} \phi_{k k k} \frac{3 !}{3 !} \cdot \frac{1}{3 !} \phi_{k k k}
$$

This term is nonzero, since the total degree of $q_{k}$ is six, which is even.

In total, after utilizing mathematical equivalences, there are five types of nonzero matrix elements with the following types of force constants, permutation factors, and reciprocal factorials: 


$$
\phi_{k l m} \phi_{k l m} \quad \frac{1}{4} \phi_{k k m} \phi_{l l m} \quad \frac{1}{4} \phi_{k k l} \phi_{k k l} \quad \frac{1}{12} \phi_{k k k} \phi_{k l l} \quad \frac{1}{36} \phi_{k k k} \phi_{k k k}
$$

Now that the types of nonzero matrix element have been identified, the possible "paths" from the initial state through a common intermediate state and back to the original state will be formulated as permitted by the harmonic oscillator selection rules.

For the first term in List (61), $\phi_{k l m} \phi_{k l m}$, each mode must change by one quantum in each off-diagonal matrix element, since the degree of each $q_{i}$ is one in each off-diagonal matrix element. There are eight possible nonzero paths in which each mode $k, l$, and $m$ can either increase or decrease, resulting in the following terms:

$$
\begin{aligned}
& h c \frac{\left\langle\mathrm{v}_{k}, \mathrm{v}_{l}, \mathrm{v}_{m}\left|\phi_{k l m} q_{k} q_{l} q_{m}\right| \mathrm{v}_{k}+1, \mathrm{v}_{l}+1, \mathrm{v}_{m}+1\right\rangle\left\langle\mathrm{v}_{k}+1, \mathrm{v}_{l}+1, \mathrm{v}_{m}+1\left|\phi_{k l m} q_{k} q_{l} q_{m}\right| \mathrm{v}_{k}, \mathrm{v}_{l}, \mathrm{v}_{m}\right\rangle}{-\omega_{k}-\omega_{l}-\omega_{m}} \\
& h c \frac{\left\langle\mathrm{v}_{k}, \mathrm{v}_{l}, \mathrm{v}_{m}\left|\phi_{k l m} q_{k} q_{l} q_{m}\right| \mathrm{v}_{k}-1, \mathrm{v}_{l}-1, \mathrm{v}_{m}-1\right\rangle\left\langle\mathrm{v}_{k}-1, \mathrm{v}_{l}-1, \mathrm{v}_{m}-1\left|\phi_{k l m} q_{k} q_{l} q_{m}\right| \mathrm{v}_{k}, \mathrm{v}_{l}, \mathrm{v}_{m}\right\rangle}{\omega_{k}+\omega_{l}+\omega_{m}} \\
& h c \frac{\left\langle\mathrm{v}_{k}, \mathrm{v}_{l}, \mathrm{v}_{m}\left|\phi_{k l m} q_{k} q_{l} q_{m}\right| \mathrm{v}_{k}+1, \mathrm{v}_{l}-1, \mathrm{v}_{m}+1\right\rangle\left\langle\mathrm{v}_{k}+1, \mathrm{v}_{l}-1, \mathrm{v}_{m}+1\left|\phi_{k l m} q_{k} q_{l} q_{m}\right| \mathrm{v}_{k}, \mathrm{v}_{l}, \mathrm{v}_{m}\right\rangle}{-\omega_{k}+\omega_{l}-\omega_{m}} \\
& h c \frac{\left\langle\mathrm{v}_{k}, \mathrm{v}_{l}, \mathrm{v}_{m}\left|\phi_{k l m} q_{k} q_{l} q_{m}\right| \mathrm{v}_{k}-1, \mathrm{v}_{l}+1, \mathrm{v}_{m}-1\right\rangle\left\langle\mathrm{v}_{k}-1, \mathrm{v}_{l}+1, \mathrm{v}_{m}-1\left|\phi_{k l m} q_{k} q_{l} q_{m}\right| \mathrm{v}_{k}, \mathrm{v}_{l}, \mathrm{v}_{m}\right\rangle}{\omega_{k}-\omega_{l}+\omega_{m}} \\
& h c \frac{\left\langle\mathrm{v}_{k}, \mathrm{v}_{l}, \mathrm{v}_{m}\left|\phi_{k l m} q_{k} q_{l} q_{m}\right| \mathrm{v}_{k}+1, \mathrm{v}_{l}+1, \mathrm{v}_{m}-1\right\rangle\left\langle\mathrm{v}_{k}+1, \mathrm{v}_{l}+1, \mathrm{v}_{m}-1\left|\phi_{k l m} q_{k} q_{l} q_{m}\right| \mathrm{v}_{k}, \mathrm{v}_{l}, \mathrm{v}_{m}\right\rangle}{-\omega_{k}-\omega_{l}+\omega_{m}} \\
& h c \frac{\left\langle\mathrm{v}_{k}, \mathrm{v}_{l}, \mathrm{v}_{m}\left|\phi_{k l m} q_{k} q_{l} q_{m}\right| \mathrm{v}_{k}-1, \mathrm{v}_{l}-1, \mathrm{v}_{m}+1\right\rangle\left\langle\mathrm{v}_{k}-1, \mathrm{v}_{l}-1, \mathrm{v}_{m}+1\left|\phi_{k l m} q_{k} q_{l} q_{m}\right| \mathrm{v}_{k}, \mathrm{v}_{l}, \mathrm{v}_{m}\right\rangle}{\omega_{k}+\omega_{l}-\omega_{m}} \\
& h c \frac{\left\langle\mathrm{v}_{k}, \mathrm{v}_{l}, \mathrm{v}_{m}\left|\phi_{k l m} q_{k} q_{l} q_{m}\right| \mathrm{v}_{k}-1, \mathrm{v}_{l}+1, \mathrm{v}_{m}+1\right\rangle\left\langle\mathrm{v}_{k}-1, \mathrm{v}_{l}+1, \mathrm{v}_{m}+1\left|\phi_{k l m} q_{k} q_{l} q_{m}\right| \mathrm{v}_{k}, \mathrm{v}_{l}, \mathrm{v}_{m}\right\rangle}{\omega_{k}-\omega_{l}-\omega_{m}} \\
& h c \frac{\left\langle\mathrm{v}_{k}, \mathrm{v}_{l}, \mathrm{v}_{m}\left|\phi_{k l m} q_{k} q_{l} q_{m}\right| \mathrm{v}_{k}+1, \mathrm{v}_{l}-1, \mathrm{v}_{m}-1\right\rangle\left\langle\mathrm{v}_{k}+1, \mathrm{v}_{l}-1, \mathrm{v}_{m}-1\left|\phi_{k l m} q_{k} q_{l} q_{m}\right| \mathrm{v}_{k}, \mathrm{v}_{l}, \mathrm{v}_{m}\right\rangle}{-\omega_{k}+\omega_{l}+\omega_{m}}
\end{aligned}
$$


The energy denominators in each of the above paths reduce to $\omega_{\mathrm{i}}$ expressions because $E=h v=h c \omega$ and the constant $h c$ in the energy denominator is cancelled out by part of the $h^{2} c^{2}$ in the numerator from the second term in Equation (57).

Combining these terms in pairs whose denominators differ by -1 , the overall expression for the $\phi_{k l m} \phi_{k l m}$ term becomes

$$
\begin{aligned}
& -h c \frac{1}{6} \sum_{k \neq l \neq m} \phi_{k l m}^{2}\left[\frac{\left\langle\mathrm{v}_{k}, \mathrm{v}_{l}, \mathrm{v}_{m}\left|q_{k} q_{l} q_{m}\right| \mathrm{v}_{k}+1, \mathrm{v}_{l}+1, \mathrm{v}_{m}+1\right\rangle^{2}-\left\langle\mathrm{v}_{k}, \mathrm{v}_{l}, \mathrm{v}_{m}\left|q_{k} q_{l} q_{m}\right| \mathrm{v}_{k}-1, \mathrm{v}_{l}-1, \mathrm{v}_{m}-1\right\rangle^{2}}{\omega_{k}+\omega_{l}+\omega_{m}}\right. \\
& +\frac{\left\langle\mathrm{v}_{k}, \mathrm{v}_{l}, \mathrm{v}_{m}\left|q_{k} q_{l} q_{m}\right| \mathrm{v}_{k}+1, \mathrm{v}_{l}-1, \mathrm{v}_{m}+1\right\rangle^{2}-\left\langle\mathrm{v}_{k}, \mathrm{v}_{l}, \mathrm{v}_{m}\left|q_{k} q_{l} q_{m}\right| \mathrm{v}_{k}-1, \mathrm{v}_{l}+1, \mathrm{v}_{m}-1\right\rangle^{2}}{\omega_{k}-\omega_{l}+\omega_{m}} \\
& \left.+\frac{\left\langle\mathrm{v}_{k}, \mathrm{v}_{l}, \mathrm{v}_{m}\left|q_{k} q_{l} q_{m}\right| \mathrm{v}_{k}+1, \mathrm{v}_{l}+1, \mathrm{v}_{m}-1\right\rangle^{2}-\left\langle\mathrm{v}_{k}, \mathrm{v}_{l}, \mathrm{v}_{m}\left|q_{k} q_{l} q_{m}\right| \mathrm{v}_{k}-1, \mathrm{v}_{l}-1, \mathrm{v}_{m}+1\right\rangle^{2}}{\omega_{k}+\omega_{l}-\omega_{m}}\right] \\
& \left.+\frac{\left\langle\mathrm{v}_{k}, \mathrm{v}_{l}, \mathrm{v}_{m}\left|q_{k} q_{l} q_{m}\right| \mathrm{v}_{k}-1, \mathrm{v}_{l}+1, \mathrm{v}_{m}+1\right\rangle^{2}-\left\langle\mathrm{v}_{k}, \mathrm{v}_{l}, \mathrm{v}_{m}\left|q_{k} q_{l} q_{m}\right| \mathrm{v}_{k}+1, \mathrm{v}_{l}-1, \mathrm{v}_{m}-1\right\rangle^{2}}{-\omega_{k}+\omega_{l}+\omega_{m}}\right]
\end{aligned}
$$

since $\left\langle\mathrm{v}_{k}\left|q_{k}\right| \mathrm{v}_{k}+1\right\rangle=\left\langle\mathrm{v}_{k}+1\left|q_{k}\right| \mathrm{v}_{k}\right\rangle$. The factor of $\frac{1}{3 !}=\frac{1}{6}$ is present to account for the $3 !=6$ ways to order three distinct indices $\mathrm{klm}$, the permutations of which would be over-counted in the non-restrictive sum. Equivalently, a restrictive sum (e.g., $k>l>m)$ could have defined the summation here without the factor of $\frac{1}{6}$.

For the second term in List (61), $\frac{1}{4} \phi_{k k m} \phi_{l l m}$, neither mode $k$ nor mode $l$ can change by more than zero quanta, since each has $q$ operators in only one off-diagonal matrix element. Also, mode $m$ can change by one quantum because the degree of $q_{m}$ is one in both offdiagonal matrix elements. Moreover, this term is not a square like the previous term is, and consequently both orderings of matrix elements, with force constants $\phi_{k k m} \phi_{l l m}$ or $\phi_{l l m} \phi_{k k m}$, must be considered. Hence, the possible nonzero paths have the following terms: 


$$
\begin{aligned}
& h c \frac{\left\langle\mathrm{v}_{k}, \mathrm{v}_{l}, \mathrm{v}_{m}\left|\phi_{k k m} q_{k}^{2} q_{m}\right| \mathrm{v}_{k}, \mathrm{v}_{l}, \mathrm{v}_{m}+1\right\rangle\left\langle\mathrm{v}_{k}, \mathrm{v}_{l}, \mathrm{v}_{m}+1\left|\phi_{l m} q_{l}^{2} q_{m}\right| \mathrm{v}_{k}, \mathrm{v}_{l}, \mathrm{v}_{m}\right\rangle}{-\omega_{m}} \\
& h c \frac{\left\langle\mathrm{v}_{k}, \mathrm{v}_{l}, \mathrm{v}_{m}\left|\phi_{k k m} q_{k}^{2} q_{m}\right| \mathrm{v}_{k}, \mathrm{v}_{l}, \mathrm{v}_{m}-1\right\rangle\left\langle\mathrm{v}_{k}, \mathrm{v}_{l}, \mathrm{v}_{m}-1\left|\phi_{l l m} q_{l}^{2} q_{m}\right| \mathrm{v}_{k}, \mathrm{v}_{l}, \mathrm{v}_{m}\right\rangle}{\omega_{m}} \\
& h c \frac{\left\langle\mathrm{v}_{k}, \mathrm{v}_{l}, \mathrm{v}_{m}\left|\phi_{l l m} q_{l}^{2} q_{m}\right| \mathrm{v}_{k}, \mathrm{v}_{l}, \mathrm{v}_{m}+1\right\rangle\left\langle\mathrm{v}_{k}, \mathrm{v}_{l}, \mathrm{v}_{m}+1\left|\phi_{k k m} q_{k}^{2} q_{m}\right| \mathrm{v}_{k}, \mathrm{v}_{l}, \mathrm{v}_{m}\right\rangle}{-\omega_{m}} \\
& h c \frac{\left\langle\mathrm{v}_{k}, \mathrm{v}_{l}, \mathrm{v}_{m}\left|\phi_{l l m} q_{l}^{2} q_{m}\right| \mathrm{v}_{k}, \mathrm{v}_{l}, \mathrm{v}_{m}-1\right\rangle\left\langle\mathrm{v}_{k}, \mathrm{v}_{l}, \mathrm{v}_{m}-1\left|\phi_{k k m} q_{k}^{2} q_{m}\right| \mathrm{v}_{k}, \mathrm{v}_{l}, \mathrm{v}_{m}\right\rangle}{\omega_{m}}
\end{aligned}
$$

The overall expression for the $\frac{1}{4} \phi_{k k m} \phi_{l l m}$ term is

$$
\begin{aligned}
& -2 \cdot \frac{1}{2 !} h c \sum_{k \neq l \neq m} \frac{1}{4} \phi_{k k m} \phi_{l l m}\left[\frac{\left\langle\mathrm{v}_{k}, \mathrm{v}_{l}, \mathrm{v}_{m}\left|q_{k}^{2} q_{m}\right| \mathrm{v}_{k}, \mathrm{v}_{l}, \mathrm{v}_{m}+1\right\rangle\left\langle\mathrm{v}_{k}, \mathrm{v}_{l}, \mathrm{v}_{m}+1\left|q_{l}^{2} q_{m}\right| \mathrm{v}_{k}, \mathrm{v}_{l}, \mathrm{v}_{m}\right\rangle}{\omega_{m}}\right. \\
& \left.-\frac{\left\langle\mathrm{v}_{k}, \mathrm{v}_{l}, \mathrm{v}_{m}\left|q_{k}^{2} q_{m}\right| \mathrm{v}_{k}, \mathrm{v}_{l}, \mathrm{v}_{m}-1\right\rangle\left\langle\mathrm{v}_{k}, \mathrm{v}_{l}, \mathrm{v}_{m}-1\left|q_{l}^{2} q_{m}\right| \mathrm{v}_{k}, \mathrm{v}_{l}, \mathrm{v}_{m}\right\rangle}{\omega_{m}}\right]
\end{aligned}
$$

The expression has a coefficient of two because, by recalling that $\phi_{k k m} \phi_{l l m}=\phi_{l l m} \phi_{k k m}$, the first path can be combined with the third, and the second path can be combined with the fourth.

The factor of $\frac{1}{2 !}$ ensures only one of each unique term is accounted for when a non-restrictive sum is used.

For the third term in List (60), $\frac{1}{4} \phi_{k k l} \phi_{k k l}$, mode $k$ can change by either zero or two quanta, since in each off-diagonal matrix element, the degree of $q_{k}$ is two, and mode $l$ must change by one since the degree of $q_{l}$ is one in both off-diagonal matrix elements. This said, the possible nonzero paths have the following terms:

$$
h c \frac{\left\langle\mathrm{v}_{k}, \mathrm{v}_{l}\left|\phi_{k k l} q_{k}^{2} q_{l}\right| \mathrm{v}_{k}+2, \mathrm{v}_{l}+1\right\rangle\left\langle\mathrm{v}_{k}+2, \mathrm{v}_{l}+1\left|\phi_{k k l} q_{k}^{2} q_{l}\right| \mathrm{v}_{k}, \mathrm{v}_{l}\right\rangle}{-2 \omega_{k}-\omega_{l}}
$$




$$
\begin{aligned}
& h c \frac{\left\langle\mathrm{v}_{k}, \mathrm{v}_{l}\left|\phi_{k k l} q_{k}^{2} q_{l}\right| \mathrm{v}_{k}-2, \mathrm{v}_{l}-1\right\rangle\left\langle\mathrm{v}_{k}-2, \mathrm{v}_{l}-1\left|\phi_{k k l} q_{k}^{2} q_{l}\right| \mathrm{v}_{k}, \mathrm{v}_{l}\right\rangle}{2 \omega_{k}+\omega_{l}} \\
& h c \frac{\left\langle\mathrm{v}_{k}, \mathrm{v}_{l}\left|\phi_{k k l} q_{k}^{2} q_{l}\right| \mathrm{v}_{k}+2, \mathrm{v}_{l}-1\right\rangle\left\langle\mathrm{v}_{k}+2, \mathrm{v}_{l}-1\left|\phi_{k k l} q_{k}^{2} q_{l}\right| \mathrm{v}_{k}, \mathrm{v}_{l}\right\rangle}{-2 \omega_{k}+\omega_{l}} \\
& h c \frac{\left\langle\mathrm{v}_{k}, \mathrm{v}_{l}\left|\phi_{k k l} q_{k}^{2} q_{l}\right| \mathrm{v}_{k}-2, \mathrm{v}_{l}+1\right\rangle\left\langle\mathrm{v}_{k}-2, \mathrm{v}_{l}+1\left|\phi_{k k l} q_{k}^{2} q_{l}\right| \mathrm{v}_{k}, \mathrm{v}_{l}\right\rangle}{2 \omega_{k}-\omega_{l}} \\
& h c \frac{\left\langle\mathrm{v}_{k}, \mathrm{v}_{l}\left|\phi_{k k l} q_{k}^{2} q_{l}\right| \mathrm{v}_{k}, \mathrm{v}_{l}+1\right\rangle\left\langle\mathrm{v}_{k}, \mathrm{v}_{l}+1\left|\phi_{k k l} q_{k}^{2} q_{l}\right| \mathrm{v}_{k}, \mathrm{v}_{l}\right\rangle}{-\omega_{l}} \\
& h c \frac{\left\langle\mathrm{v}_{k}, \mathrm{v}_{l}\left|\phi_{k k l} q_{k}^{2} q_{l}\right| \mathrm{v}_{k}, \mathrm{v}_{l}-1\right\rangle\left\langle\mathrm{v}_{k}, \mathrm{v}_{l}-1\left|\phi_{k k l} q_{k}^{2} q_{l}\right| \mathrm{v}_{k}, \mathrm{v}_{l}\right\rangle}{\omega_{l}}
\end{aligned}
$$

Combining the terms whose denominators differ by -1 , the overall expression for the $\frac{1}{4} \phi_{k k l} \phi_{k k l}$ term is

$$
\begin{aligned}
& -h c \sum_{k \neq l} \frac{1}{4} \phi_{k k l}^{2}\left[\frac{\left\langle\mathrm{v}_{k}, \mathrm{v}_{l}\left|q_{k}^{2} q_{l}\right| \mathrm{v}_{k}+2, \mathrm{v}_{l}+1\right\rangle^{2}-\left\langle\mathrm{v}_{k}, \mathrm{v}_{l}\left|q_{k}^{2} q_{l}\right| \mathrm{v}_{k}-2, \mathrm{v}_{l}-1\right\rangle^{2}}{2 \omega_{k}+\omega_{l}}\right. \\
& +\frac{\left\langle\mathrm{v}_{k}, \mathrm{v}_{l}\left|q_{k}^{2} q_{l}\right| \mathrm{v}_{k}+2, \mathrm{v}_{l}-1\right\rangle^{2}-\left\langle\mathrm{v}_{k}, \mathrm{v}_{l}\left|q_{k}^{2} q_{l}\right| \mathrm{v}_{k}-2, \mathrm{v}_{l}+1\right\rangle^{2}}{2 \omega_{k}-\omega_{l}} \\
& \left.+\frac{\left\langle\mathrm{v}_{k}, \mathrm{v}_{l}\left|q_{k}^{2} q_{l}\right| \mathrm{v}_{k}, \mathrm{v}_{l}+1\right\rangle^{2}-\left\langle\mathrm{v}_{k}, \mathrm{v}_{l}\left|q_{k}^{2} q_{l}\right| \mathrm{v}_{k}, \mathrm{v}_{l}-1\right\rangle^{2}}{\omega_{l}}\right]
\end{aligned}
$$

No factors for non-restrictive summations are included in these matrix elements.

Unlike the previous two matrix elements, this type lacks symmetry. The symmetry present in the first two allow summation elements to occur multiple times. Due to the asymmetry of this matrix element type, the non-restrictive sum includes each unique term only once.

For the fourth term in List (61), $\frac{1}{12} \phi_{k k k} \phi_{k l l}$, mode $k$ only can change by one quantum because, if it changes by three quanta, the matrix element involving $\phi_{k l l}$ would be zero. Similarly, mode $l$ can only change by zero quanta, since the degree of $q_{l}$ is zero in the matrix 
element involving $\phi_{k k k}$. In addition, this term is not a square, and consequently both orderings of matrix elements, with force constants $\phi_{k k k} \phi_{k l l}$ or $\phi_{k l l} \phi_{k k k}$, must be considered. These observations considered, the possible nonzero paths have the following terms:

$$
\begin{aligned}
& h c \frac{\left\langle\mathrm{v}_{k}, \mathrm{v}_{l}\left|\phi_{k k k} q_{k}^{3}\right| \mathrm{v}_{k}+1, \mathrm{v}_{l}\right\rangle\left\langle\mathrm{v}_{k}+1, \mathrm{v}_{l}\left|\phi_{k l l} q_{k} q_{l}^{2}\right| \mathrm{v}_{k}, \mathrm{v}_{l}\right\rangle}{-\omega_{k}} \\
& h c \frac{\left\langle\mathrm{v}_{k}, \mathrm{v}_{l}\left|\phi_{k k k} q_{k}^{3}\right| \mathrm{v}_{k}-1, \mathrm{v}_{l}\right\rangle\left\langle\mathrm{v}_{k}-1, \mathrm{v}_{l}\left|\phi_{k l l} q_{k} q_{l}^{2}\right| \mathrm{v}_{k}, \mathrm{v}_{l}\right\rangle}{\omega_{k}} \\
& h c \frac{\left\langle\mathrm{v}_{k}, \mathrm{v}_{l}\left|\phi_{k l l} q_{k} q_{l}^{2}\right| \mathrm{v}_{k}+1, \mathrm{v}_{l}\right\rangle\left\langle\mathrm{v}_{k}+1, \mathrm{v}_{l}\left|\phi_{k k k} q_{k}^{3}\right| \mathrm{v}_{k}, \mathrm{v}_{l}\right\rangle}{-\omega_{k}} \\
& h c \frac{\left\langle\mathrm{v}_{k}, \mathrm{v}_{l}\left|\phi_{k l l} q_{k} q_{l}^{2}\right| \mathrm{v}_{k}-1, \mathrm{v}_{l}\right\rangle\left\langle\mathrm{v}_{k}-1, \mathrm{v}_{l}\left|\phi_{k k k} q_{k}^{3}\right| \mathrm{v}_{k}, \mathrm{v}_{l}\right\rangle}{\omega_{k}}
\end{aligned}
$$

The overall expression for the $\frac{1}{12} \phi_{k k k} \phi_{k l l}$ term is

$$
-2 h c \sum_{k \neq l} \frac{1}{12} \phi_{k k k} \phi_{k l l}\left[\frac{\left\langle\mathrm{v}_{k}, \mathrm{v}_{l}\left|q_{k}^{3}\right| \mathrm{v}_{k}+1, \mathrm{v}_{l}\right\rangle\left\langle\mathrm{v}_{k}+1, \mathrm{v}_{l}\left|q_{k} q_{l}^{2}\right| \mathrm{v}_{k}, \mathrm{v}_{l}\right\rangle-\left\langle\mathrm{v}_{k}, \mathrm{v}_{l}\left|q_{k}^{3}\right| \mathrm{v}_{k}-1, \mathrm{v}_{l}\right\rangle\left\langle\mathrm{v}_{k}-1, \mathrm{v}_{l}\left|q_{k} q_{l}^{2}\right| \mathrm{v}_{k}, \mathrm{v}_{l}\right\rangle}{\omega_{k}}\right]
$$

As in the second matrix element type, this expression includes a coefficient of two obtained by combining the first path with the third and the second path with the fourth. As in the third matrix element type, these matrix elements do not require a factor for non-restrictive summation due to their asymmetry.

For the last term in List (61), $\frac{1}{36} \phi_{k k k}^{2}$, mode $k$ can change by either one or three quanta, since the degree of $q_{k}$ is three in each off-diagonal matrix element. The possible nonzero paths have the following terms:

$$
h c \frac{\left\langle\mathrm{v}_{k}\left|\phi_{k k k} q_{k}^{3}\right| \mathrm{v}_{k}+3\right\rangle\left\langle\mathrm{v}_{k}+3\left|\phi_{k k k} q_{k}^{3}\right| \mathrm{v}_{k}\right\rangle}{-3 \omega_{k}}
$$




$$
\begin{gathered}
h c \frac{\left\langle\mathrm{v}_{k}\left|\phi_{k k k} q_{k}^{3}\right| \mathrm{v}_{k}-3\right\rangle\left\langle\mathrm{v}_{k}-3\left|\phi_{k k k} q_{k}^{3}\right| \mathrm{v}_{k}\right\rangle}{3 \omega_{k}} \\
h c \frac{\left\langle\mathrm{v}_{k}\left|\phi_{k k k} q_{k}^{3}\right| \mathrm{v}_{k}+1\right\rangle\left\langle\mathrm{v}_{k}+1\left|\phi_{k k k} q_{k}^{3}\right| \mathrm{v}_{k}\right\rangle}{-\omega_{k}} \\
h c \frac{\left\langle\mathrm{v}_{k}\left|\phi_{k k k} q_{k}^{3}\right| \mathrm{v}_{k}-1\right\rangle\left\langle\mathrm{v}_{k}-1\left|\phi_{k k k} q_{k}^{3}\right| \mathrm{v}_{k}\right\rangle}{\omega_{k}}
\end{gathered}
$$

Combining these terms, the overall expression for the $\frac{1}{36} \phi_{k k k}^{2}$ term becomes

$$
-h c \sum_{k} \frac{1}{36} \phi_{k k k}^{2}\left[\frac{\left\langle\mathrm{v}_{k}\left|q_{k}^{3}\right| \mathrm{v}_{k}+3\right\rangle^{2}-\left\langle\mathrm{v}_{k}\left|q_{k}^{3}\right| \mathrm{v}_{k}-3\right\rangle^{2}}{3 \omega_{k}}+\frac{\left\langle\mathrm{v}_{k}\left|q_{k}^{3}\right| \mathrm{v}_{k}+1\right\rangle^{2}-\left\langle\mathrm{v}_{k}\left|q_{k}^{3}\right| \mathrm{v}_{k}-1\right\rangle^{2}}{\omega_{k}}\right]
$$

Since a single sum is both restrictive and non-restrictive, no restriction factor need be included in the expression.

Adding the five overall expressions together, the overall second part of Equation (57) becomes

$$
\begin{aligned}
h^{2} c^{2} \sum_{\gamma \neq a} \frac{\left\langle a\left|\sum_{k l m} \frac{1}{3 !} \phi_{k l m} q_{k} q_{l} q_{m}\right| \gamma\right\rangle\left\langle\gamma\left|\sum_{k^{\prime} l^{\prime} m^{3}} \frac{1}{3 !} \phi_{k^{\prime} l^{\prime} m^{\prime}} q_{k^{\prime}} q_{l^{\prime}} q_{m^{\prime}}\right| a\right\rangle}{E_{a}^{o}-E_{\gamma}^{o}}= \\
-h c \sum_{k \neq l \neq m} \frac{1}{6} \phi_{k l m}^{2}\left[\frac{\left\langle\mathrm{v}_{k}, \mathrm{v}_{l}, \mathrm{v}_{m}\left|q_{k} q_{l} q_{m}\right| \mathrm{v}_{k}+1, \mathrm{v}_{l}+1, \mathrm{v}_{m}+1\right\rangle^{2}-\left\langle\mathrm{v}_{k}, \mathrm{v}_{l}, \mathrm{v}_{m}\left|q_{k} q_{l} q_{m}\right| \mathrm{v}_{k}-1, \mathrm{v}_{l}-1, \mathrm{v}_{m}-1\right\rangle^{2}}{\omega_{k}+\omega_{l}+\omega_{m}}\right. \\
+\frac{\left\langle\mathrm{v}_{k}, \mathrm{v}_{l}, \mathrm{v}_{m}\left|q_{k} q_{l} q_{m}\right| \mathrm{v}_{k}+1, \mathrm{v}_{l}-1, \mathrm{v}_{m}+1\right\rangle^{2}-\left\langle\mathrm{v}_{k}, \mathrm{v}_{l}, \mathrm{v}_{m}\left|q_{k} q_{l} q_{m}\right| \mathrm{v}_{k}-1, \mathrm{v}_{l}+1, \mathrm{v}_{m}-1\right\rangle^{2}}{\omega_{k}-\omega_{l}+\omega_{m}} \\
+\frac{\left\langle\mathrm{v}_{k}, \mathrm{v}_{l}, \mathrm{v}_{m}\left|q_{k} q_{l} q_{m}\right| \mathrm{v}_{k}+1, \mathrm{v}_{l}+1, \mathrm{v}_{m}-1\right\rangle^{2}-\left\langle\mathrm{v}_{k}, \mathrm{v}_{l}, \mathrm{v}_{m}\left|q_{k} q_{l} q_{m}\right| \mathrm{v}_{k}-1, \mathrm{v}_{l}-1, \mathrm{v}_{m}+1\right\rangle^{2}}{\omega_{k}+\omega_{l}-\omega_{m}} \\
+\frac{\left\langle\mathrm{v}_{k}, \mathrm{v}_{l}, \mathrm{v}_{m}\left|q_{k} q_{l} q_{m}\right| \mathrm{v}_{k}-1, \mathrm{v}_{l}+1, \mathrm{v}_{m}+1\right\rangle^{2}-\left\langle\mathrm{v}_{k}, \mathrm{v}_{l}, \mathrm{v}_{m}\left|q_{k} q_{l} q_{m}\right| \mathrm{v}_{k}+1, \mathrm{v}_{l}-1, \mathrm{v}_{m}-1\right\rangle^{2}}{-\omega_{k}+\omega_{l}+\omega_{m}}
\end{aligned}
$$




$$
\begin{aligned}
&-h c \sum_{k \neq l \neq m} \frac{1}{4} \phi_{k k m} \phi_{l l m}\left[\frac{\left\langle\mathrm{v}_{k}, \mathrm{v}_{l}, \mathrm{v}_{m}\left|q_{k}^{2} q_{m}\right| \mathrm{v}_{k}, \mathrm{v}_{l}, \mathrm{v}_{m}+1\right\rangle\left\langle\mathrm{v}_{k}, \mathrm{v}_{l}, \mathrm{v}_{m}+1\left|q_{l}^{2} q_{m}\right| \mathrm{v}_{k}, \mathrm{v}_{l}, \mathrm{v}_{m}\right\rangle}{\omega_{m}}\right. \\
&\left.-\frac{\left\langle\mathrm{v}_{k}, \mathrm{v}_{l}, \mathrm{v}_{m}\left|q_{k}^{2} q_{m}\right| \mathrm{v}_{k}, \mathrm{v}_{l}, \mathrm{v}_{m}-1\right\rangle\left\langle\mathrm{v}_{k}, \mathrm{v}_{l}, \mathrm{v}_{m}-1\left|q_{l}^{2} q_{m}\right| \mathrm{v}_{k}, \mathrm{v}_{l}, \mathrm{v}_{m}\right\rangle}{\omega_{m}}\right] \\
&-h c \sum_{k \neq l} \frac{1}{4} \phi_{k k l}^{2}\left[\frac{\left\langle\mathrm{v}_{k}, \mathrm{v}_{l}\left|q_{k}^{2} q_{l}\right| \mathrm{v}_{k}+2, \mathrm{v}_{l}+1\right\rangle^{2}-\left\langle\mathrm{v}_{k}, \mathrm{v}_{l}\left|q_{k}^{2} q_{l}\right| \mathrm{v}_{k}-2, \mathrm{v}_{l}-1\right\rangle^{2}}{2 \omega_{k}+\omega_{l}}\right. \\
&+\frac{\left\langle\mathrm{v}_{k}, \mathrm{v}_{l}\left|q_{k}^{2} q_{l}\right| \mathrm{v}_{k}+2, \mathrm{v}_{l}-1\right\rangle^{2}-\left\langle\mathrm{v}_{k}, \mathrm{v}_{l}\left|q_{k}^{2} q_{l}\right| \mathrm{v}_{k}-2, \mathrm{v}_{l}+1\right\rangle^{2}}{2 \omega_{k}-\omega_{l}} \\
&\left.+\frac{\left.\left\langle\mathrm{v}_{k}, \mathrm{v}_{l}\left|q_{k}^{2} q_{l}\right| \mathrm{v}_{k}, \mathrm{v}_{l}+1\right\rangle^{2}-\left\langle\mathrm{v}_{k}, \mathrm{v}_{l}\left|q_{k}^{2} q_{l}\right| \mathrm{v}_{k}, \mathrm{v}_{l}-1\right\rangle^{2}\right]}{\omega_{l}}\right] \\
&-h c \sum_{k \neq l} \frac{1}{6} \phi_{k k k} \phi_{k l l}\left[\frac{\left\langle\mathrm{v}_{k}, \mathrm{v}_{l}\left|q_{k}^{3}\right| \mathrm{v}_{k}+1, \mathrm{v}_{l}\right\rangle\left\langle\mathrm{v}_{k}+1, \mathrm{v}_{l}\left|q_{k} q_{l}^{2}\right| \mathrm{v}_{k}, \mathrm{v}_{l}\right\rangle-\left\langle\mathrm{v}_{k}, \mathrm{v}_{l}\left|q_{k}^{3}\right| \mathrm{v}_{k}-1, \mathrm{v}_{l}\right\rangle\left\langle\mathrm{v}_{k}-1, \mathrm{v}_{l}\left|q_{k} q_{l}^{2}\right| \mathrm{v}_{k}, \mathrm{v}_{l}\right\rangle}{\omega_{k}}\right] \\
&-h c \sum_{k} \frac{1}{36} \phi_{k k k}^{2}\left[\frac{\left\langle\mathrm{v}_{k}\left|q_{k}^{3}\right| \mathrm{v}_{k}+3\right\rangle^{2}-\left\langle\mathrm{v}_{k}\left|q_{k}^{3}\right| \mathrm{v}_{k}-3\right\rangle^{2}}{3 \omega_{k}}+\frac{\left\langle\mathrm{v}_{k}\left|q_{k}^{3}\right| \mathrm{v}_{k}+1\right\rangle^{2}-\left\langle\mathrm{v}_{k}\left|q_{k}^{3}\right| \mathrm{v}_{k}-1\right\rangle^{2}}{\omega_{k}}\right]
\end{aligned}
$$

To convert Equation (62) to a more concise form, the matrix elements must be evaluated and then combined. This "simplification" process requires a great deal of algebraic manipulation, which was performed by hand and verified using Maple. The Maple output is presented in the supplementary material. The final expression can be written as

$$
h^{2} c^{2} \sum_{\gamma \neq a} \frac{\left\langle a\left|\sum_{k l m} \frac{1}{3 !} \phi_{k l m} q_{k} q_{l} q_{m}\right| \gamma\right\rangle\left\langle\gamma\left|\sum_{k^{\prime} l^{\prime} m^{\prime}} \frac{1}{3 !} \phi_{k^{\prime} l^{\prime} m^{\prime}} q_{k^{\prime}} q_{l^{\prime}} q_{m^{\prime}}\right| a\right\rangle}{E_{a}^{o}-E_{\gamma}^{o}}=
$$




$$
\begin{aligned}
& -h c \sum_{k \neq l \neq m} \frac{\phi_{k l m}^{2}}{12 N_{k l m}} \omega_{m}\left(\omega_{m}^{2}-\omega_{l}^{2}-\omega_{k}^{2}\right)\left(\mathrm{v}_{k}+\frac{1}{2}\right)\left(\mathrm{v}_{l}+\frac{1}{2}\right)-h c \sum_{k \neq l \neq m} \frac{\phi_{k l m}^{2}}{12 N_{k l m}} \omega_{l}\left(\omega_{l}^{2}-\omega_{k}^{2}-\omega_{m}^{2}\right)\left(\mathrm{v}_{k}+\frac{1}{2}\right)\left(\mathrm{v}_{m}+\frac{1}{2}\right) \\
& -h c \sum_{k \neq l \neq m} \frac{\phi_{k l m}^{2}}{12 N_{k l m}} \omega_{k}\left(\omega_{k}^{2}-\omega_{l}^{2}-\omega_{m}^{2}\right)\left(\mathrm{v}_{l}+\frac{1}{2}\right)\left(\mathrm{v}_{m}+\frac{1}{2}\right)-h c \sum_{k \neq l \neq m} \frac{\phi_{k l m}^{2}}{24 N_{k l m}} \omega_{k} \omega_{l} \omega_{m} \\
& -h c \sum_{k \neq l \neq m} \frac{1}{8} \phi_{k k m} \phi_{l l m} \frac{1}{\omega_{m}}\left(\mathrm{v}_{k}+\frac{1}{2}\right)\left(\mathrm{v}_{l}+\frac{1}{2}\right)-h c \sum_{k \neq l} \frac{\phi_{k k l}^{2}}{2} \frac{\omega_{k}}{\left(4 \omega_{k}^{2}-\omega_{l}^{2}\right)}\left(\mathrm{v}_{k}+\frac{1}{2}\right)\left(\mathrm{v}_{l}+\frac{1}{2}\right) \\
& -h c \sum_{k \neq l} \frac{1}{16} \phi_{k k l}^{2} \frac{\left(8 \omega_{k}^{2}-3 \omega_{l}^{2}\right)}{\omega_{l}\left(4 \omega_{k}^{2}-\omega_{l}^{2}\right)}\left(\mathrm{v}_{k}+\frac{1}{2}\right)^{2}+h c \sum_{k \neq l} \frac{3}{64} \phi_{k k l}^{2} \frac{\omega_{l}}{\left(4 \omega_{k}^{2}-\omega_{l}^{2}\right)} \\
& -h c \sum_{k \neq l} \frac{1}{4} \phi_{k k k} \phi_{k l l} \frac{1}{\omega_{k}}\left(\mathrm{v}_{k}+\frac{1}{2}\right)\left(\mathrm{v}_{l}+\frac{1}{2}\right)-h c \sum_{k} \frac{5}{48} \phi_{k k k}^{2} \frac{1}{\omega_{k}}\left(\mathrm{v}_{k}+\frac{1}{2}\right)^{2}-h c \sum_{k} \frac{7}{576} \phi_{k k k}^{2}
\end{aligned}
$$

where

$$
N_{k l m}=\left(\omega_{k}+\omega_{l}+\omega_{m}\right)\left(\omega_{k}-\omega_{l}-\omega_{m}\right)\left(\omega_{k}-\omega_{l}+\omega_{m}\right)\left(\omega_{k}+\omega_{l}-\omega_{m}\right)
$$

Equation (63) is encoded in FORTRAN programs included in the supplemental material.

By arranging the terms according to their $\mathrm{v}_{\mathrm{i}}$ expressions, Equation (63) becomes

$$
\begin{aligned}
& h^{2} c^{2} \sum_{\gamma \neq a} \frac{\left\langle a\left|\sum_{k l m} \frac{1}{3 !} \phi_{k l m} q_{k} q_{l} q_{m}\right| \gamma\right\rangle\left\langle\gamma\left|\sum_{k^{\prime} l^{\prime} m^{3}} \frac{1}{3 !} \phi_{k^{\prime} l^{\prime} m^{\prime}} q_{k^{\prime}} q_{l^{\prime}} q_{m^{\prime}}\right| a\right\rangle}{E_{a}^{o}-E_{\gamma}^{o}}= \\
& -h c \sum_{k \neq l} \frac{1}{16} \phi_{k k l}^{2} \frac{\left(8 \omega_{k}^{2}-3 \omega_{l}^{2}\right)}{\omega_{l}\left(4 \omega_{k}^{2}-\omega_{l}^{2}\right)}\left(\mathrm{v}_{k}+\frac{1}{2}\right)^{2}-h c \sum_{k} \frac{5}{48} \phi_{k k k}^{2} \frac{1}{\omega_{k}}\left(\mathrm{v}_{k}+\frac{1}{2}\right)^{2} \\
& -h c \sum_{k \neq l \neq m} \frac{1}{8} \phi_{k k m} \phi_{l l m} \frac{1}{\omega_{m}}\left(\mathrm{v}_{k}+\frac{1}{2}\right)\left(\mathrm{v}_{l}+\frac{1}{2}\right)-h c \sum_{k \neq l} \frac{1}{4} \phi_{k k k} \phi_{k l l} \frac{1}{\omega_{k}}\left(\mathrm{v}_{k}+\frac{1}{2}\right)\left(\mathrm{v}_{l}+\frac{1}{2}\right) \\
& -h c \sum_{k \neq l \neq m} \frac{\phi_{k l m}^{2}}{12 N_{k l m}} \omega_{m}\left(\omega_{m}^{2}-\omega_{l}^{2}-\omega_{k}^{2}\right)\left(\mathrm{v}_{k}+\frac{1}{2}\right)\left(\mathrm{v}_{l}+\frac{1}{2}\right)-h c \sum_{k \neq l \neq m} \frac{\phi_{k l m}^{2}}{12 N_{k l m}} \omega_{l}\left(\omega_{l}^{2}-\omega_{k}^{2}-\omega_{m}^{2}\right)\left(\mathrm{v}_{k}+\frac{1}{2}\right)\left(\mathrm{v}_{m}+\frac{1}{2}\right) \\
& -h c \sum_{k \neq l \neq m} \frac{\phi_{k l m}^{2}}{12 N_{k l m}} \omega_{k}\left(\omega_{k}^{2}-\omega_{l}^{2}-\omega_{m}^{2}\right)\left(\mathrm{v}_{l}+\frac{1}{2}\right)\left(\mathrm{v}_{m}+\frac{1}{2}\right)-h c \sum_{k \neq l} \frac{\phi_{k k l}^{2}}{2} \frac{\omega_{k}}{\left(4 \omega_{k}^{2}-\omega_{l}^{2}\right)}\left(\mathrm{v}_{k}+\frac{1}{2}\right)\left(\mathrm{v}_{l}+\frac{1}{2}\right) \\
& -h c \sum_{k \neq l \neq m} \frac{\phi_{k l m}^{2}}{24 N_{k l m}} \omega_{k} \omega_{l} \omega_{m}+h c \sum_{k \neq l} \frac{3}{64} \phi_{k k l}^{2} \frac{\omega_{l}}{\left(4 \omega_{k}^{2}-\omega_{l}^{2}\right)}-h c \sum_{k} \frac{7}{576} \phi_{k k k}^{2}
\end{aligned}
$$

Now some conditions on the summations will be removed so that terms in Equation (65) of similar forms can be collapsed into more general summations. 
Both terms containing $\left(\mathrm{v}_{k}+\frac{1}{2}\right)^{2}$ can be combined into one summation

$$
-h c \sum_{k l} \frac{1}{16} \phi_{k k l}^{2} \frac{\left(8 \omega_{k}^{2}-3 \omega_{l}^{2}\right)}{\omega_{l}\left(4 \omega_{k}^{2}-\omega_{l}^{2}\right)}\left(\mathrm{v}_{k}+\frac{1}{2}\right)^{2}
$$

since the second term containing $\left(\mathrm{v}_{k}+\frac{1}{2}\right)^{2}$ is equivalent to the first term where $l=k$

$$
\begin{aligned}
& -h c \sum_{k} \frac{1}{16} \phi_{k k k}^{2} \frac{\left(8 \omega_{k}^{2}-3 \omega_{k}^{2}\right)}{\omega_{k}\left(4 \omega_{k}^{2}-\omega_{k}^{2}\right)}\left(\mathrm{v}_{k}+\frac{1}{2}\right)^{2} \\
& =-h c \sum_{k} \frac{1}{16} \phi_{k k k}^{2} \frac{5 \omega_{k}^{2}}{\omega_{k}\left(3 \omega_{k}^{2}\right)}\left(\mathrm{v}_{k}+\frac{1}{2}\right)^{2} \\
& =-h c \sum_{k} \frac{5}{48} \phi_{k k k}^{2} \frac{1}{\omega_{k}}\left(\mathrm{v}_{k}+\frac{1}{2}\right)^{2}
\end{aligned}
$$

Also, the first two terms containing $\left(\mathrm{v}_{k}+\frac{1}{2}\right)\left(\mathrm{v}_{l}+\frac{1}{2}\right)$, the third and fourth terms in Equation (65), can be combined into one summation of the general form

$$
-h c \sum_{k \neq l, m} \frac{1}{8} \phi_{k k m} \phi_{l l m} \frac{1}{\omega_{m}}\left(\mathrm{v}_{k}+\frac{1}{2}\right)\left(\mathrm{v}_{l}+\frac{1}{2}\right)
$$

since the fourth term occurs when $m=k$

$$
\begin{aligned}
& -h c \sum_{k \neq l} \frac{1}{8} \phi_{k k k} \phi_{l l k} \frac{1}{\omega_{k}}\left(\mathrm{v}_{k}+\frac{1}{2}\right)\left(\mathrm{v}_{l}+\frac{1}{2}\right) \\
& =-h c \sum_{k \neq l} \frac{1}{8} \phi_{k k k} \phi_{k l l} \frac{1}{\omega_{k}}\left(\mathrm{v}_{k}+\frac{1}{2}\right)\left(\mathrm{v}_{l}+\frac{1}{2}\right)
\end{aligned}
$$

or when $m=l$

$$
\begin{aligned}
& -h c \sum_{k \neq l} \frac{1}{8} \phi_{k k l} \phi_{l l l} \frac{1}{\omega_{k}}\left(\mathrm{v}_{k}+\frac{1}{2}\right)\left(\mathrm{v}_{l}+\frac{1}{2}\right) \\
& =-h c \sum_{k \neq l} \frac{1}{8} \phi_{k k l} \phi_{l l l} \frac{1}{\omega_{k}}\left(\mathrm{v}_{k}+\frac{1}{2}\right)\left(\mathrm{v}_{l}+\frac{1}{2}\right)
\end{aligned}
$$

Note that the fourth term where $m=l$ is a mapping of $k$ and $l$ onto $l$ and $k$, where $k$ and $l$ are allowed to run over all possible indices. These two sums give identical results, and the fourth 
term includes both of them by writing the terms using the first convention $(m=k)$ and including a factor of two.

Notice the fifth, sixth, and seventh terms in Equation (65) have similar forms despite their disparate $\mathrm{v}_{\mathrm{i}} \mathrm{v}_{\mathrm{j}}$ dependences. Additionally, the eighth term also has a similar form. These terms will be combined into the summation of the general form

$$
-h c \sum_{k \neq l, m} \frac{\phi_{k l m}^{2}}{4 N_{k l m}} \omega_{m}\left(\omega_{m}^{2}-\omega_{l}^{2}-\omega_{k}^{2}\right)\left(\mathrm{v}_{k}+\frac{1}{2}\right)\left(\mathrm{v}_{l}+\frac{1}{2}\right)
$$

In the absence of restrictions, the fifth, sixth, and seventh terms are equivalent, where the three involve different mappings of the indices $k, l$, and $m$. Thus, the fifth term can be multiplied by three to give

$$
-h c \sum_{k \neq l \neq m} \frac{\phi_{k l m}^{2}}{4 N_{k l m}} \omega_{m}\left(\omega_{m}^{2}-\omega_{l}^{2}-\omega_{k}^{2}\right)\left(\mathrm{v}_{k}+\frac{1}{2}\right)\left(\mathrm{v}_{l}+\frac{1}{2}\right)
$$

The eighth term allows the restrictions on $m$ to be removed from the above expression. When $m=k$ in the general term, it is clear that part of the eighth term follows

$$
\begin{aligned}
& -h c \sum_{k \neq l, m} \frac{\phi_{k l k}^{2}}{4 N_{k l k}} \omega_{k}\left(\omega_{k}^{2}-\omega_{l}^{2}-\omega_{k}^{2}\right)\left(\mathrm{v}_{k}+\frac{1}{2}\right)\left(\mathrm{v}_{l}+\frac{1}{2}\right) \\
& =-h c \sum_{k \neq l} \frac{\phi_{k l k}^{2}}{4} \frac{\omega_{k}\left(-\omega_{l}^{2}\right)}{\left(\omega_{k}+\omega_{l}+\omega_{k}\right)\left(\omega_{k}-\omega_{l}-\omega_{k}\right)\left(\omega_{k}-\omega_{l}+\omega_{k}\right)\left(\omega_{k}+\omega_{l}-\omega_{k}\right)}\left(\mathrm{v}_{k}+\frac{1}{2}\right)\left(\mathrm{v}_{l}+\frac{1}{2}\right) \\
& =-h c \sum_{k \neq l} \frac{\phi_{k k l}^{2}}{4} \frac{\omega_{k}\left(-\omega_{l}^{2}\right)}{\left(2 \omega_{k}+\omega_{l}\right)\left(-\omega_{l}\right)\left(2 \omega_{k}-\omega_{l}\right)\left(\omega_{l}\right)}\left(\mathrm{v}_{k}+\frac{1}{2}\right)\left(\mathrm{v}_{l}+\frac{1}{2}\right) \\
& =-h c \sum_{k \neq l} \frac{\phi_{k k l}^{2}}{4} \frac{\omega_{k}}{\left(4 \omega_{k}^{2}-\omega_{l}^{2}\right)}\left(\mathrm{v}_{k}+\frac{1}{2}\right)\left(\mathrm{v}_{l}+\frac{1}{2}\right)
\end{aligned}
$$

Similarly, when $m=l$ in the general term, the rest of the eighth term follows 


$$
\begin{aligned}
& -h c \sum_{k \neq l, m} \frac{\phi_{k l l}^{2}}{4 N_{k l l}} \omega_{l}\left(\omega_{l}^{2}-\omega_{l}^{2}-\omega_{k}^{2}\right)\left(\mathrm{v}_{k}+\frac{1}{2}\right)\left(\mathrm{v}_{l}+\frac{1}{2}\right) \\
& =-h c \sum_{k \neq l} \frac{\phi_{k l l}^{2}}{4} \frac{\omega_{l}\left(-\omega_{k}^{2}\right)}{\left(\omega_{k}+\omega_{l}+\omega_{l}\right)\left(\omega_{k}-\omega_{l}-\omega_{l}\right)\left(\omega_{k}-\omega_{l}+\omega_{l}\right)\left(\omega_{k}+\omega_{l}-\omega_{l}\right)}\left(\mathrm{v}_{k}+\frac{1}{2}\right)\left(\mathrm{v}_{l}+\frac{1}{2}\right) \\
& =-h c \sum_{k \neq l} \frac{\phi_{k l l}^{2}}{4} \frac{\omega_{l}\left(-\omega_{k}^{2}\right)}{\left(2 \omega_{l}+\omega_{k}\right)\left(\omega_{k}-2 \omega_{l}\right)\left(\omega_{k}\right)\left(\omega_{k}\right)}\left(\mathrm{v}_{k}+\frac{1}{2}\right)\left(\mathrm{v}_{l}+\frac{1}{2}\right) \\
& =-h c \sum_{k \neq l} \frac{\phi_{k l l}^{2}}{4} \frac{\omega_{l}}{\left(4 \omega_{l}^{2}-\omega_{k}^{2}\right)}\left(\mathrm{v}_{k}+\frac{1}{2}\right)\left(\mathrm{v}_{l}+\frac{1}{2}\right)
\end{aligned}
$$

Note that these two instances account for all terms in the non-restrictive sums over $k$ and $l$ given in the general term and that, like in the previous combination of summations, these two sums are identical. In the representation of the eighth term in Equation (65), the first convention $(m=k)$ has been used, and a factor of two has been included to account for all possible terms.

These simplifications considered, the terms of Equation (65) are, with the addition of the all quartic terms from Equation (60)

$$
\begin{aligned}
& h c\left\langle a\left|\sum_{k l m n} \frac{1}{4 !} \phi_{k l m n} q_{k} q_{l} q_{m} q_{n}\right| a\right\rangle+h^{2} c^{2} \sum_{\gamma \neq a} \frac{\left\langle a\left|\sum_{k l m} \frac{1}{3 !} \phi_{k l m} q_{k} q_{l} q_{m}\right| \gamma\right\rangle\left\langle\gamma\left|\sum_{k^{\prime} l^{\prime} \cdot} \frac{1}{3 !} \phi_{k^{\prime} l^{\prime} m^{\prime}} q_{k^{\prime}} q_{l^{\prime}} q_{m^{\prime}}\right| a\right\rangle}{E_{a}^{o}-E_{\gamma}^{o}}= \\
& \frac{1}{16} h c \sum_{k} \phi_{k k k k}\left[\left(\mathrm{v}_{k}+\frac{1}{2}\right)^{2}+\frac{1}{4}\right]+\frac{1}{8} h c \sum_{k \neq l} \phi_{k k l l}\left(\mathrm{v}_{k}+\frac{1}{2}\right)\left(\mathrm{v}_{l}+\frac{1}{2}\right)-h c \sum_{k l} \frac{1}{16} \phi_{k k l}^{2} \frac{\left(8 \omega_{k}^{2}-3 \omega_{l}^{2}\right)}{\omega_{l}\left(4 \omega_{k}^{2}-\omega_{l}^{2}\right)}\left(\mathrm{v}_{k}+\frac{1}{2}\right)^{2} \\
& -h c \sum_{k \neq l, m} \frac{1}{8} \phi_{k k m} \phi_{l l m} \frac{1}{\omega_{m}}\left(\mathrm{v}_{k}+\frac{1}{2}\right)\left(\mathrm{v}_{l}+\frac{1}{2}\right)-h c \sum_{k \neq l, m} \frac{\phi_{k l m}^{2}}{4 N_{k l m}} \omega_{m}\left(\omega_{m}^{2}-\omega_{l}^{2}-\omega_{k}^{2}\right)\left(\mathrm{v}_{k}+\frac{1}{2}\right)\left(\mathrm{v}_{l}+\frac{1}{2}\right) \\
& -h c \sum_{k \neq l \neq m} \frac{\phi_{k l m}^{2}}{24 N_{k l m}} \omega_{k} \omega_{l} \omega_{m}+h c \sum_{k \neq l} \frac{3}{64} \phi_{k k l}^{2} \frac{\omega_{l}}{\left(4 \omega_{k}^{2}-\omega_{l}^{2}\right)}-h c \sum_{k} \frac{7}{576} \phi_{k k k}^{2}
\end{aligned}
$$

\section{The vibrational angular momentum term}

Here the derivation of the second order energy correction diagonal matrix elements in IV.A.4 is extended to include vibrational angular momentum and to derive the terms containing Coriolis coupling constants. 
In Equations (2) and (45), the vibrational angular momentum term in the Hamiltonian is $\frac{1}{2} \sum_{\alpha \beta} \mu_{\alpha \beta} \hat{\pi}_{\alpha} \hat{\pi}_{\beta}$, and the Coriolis coupling operator $\hat{\pi}_{\alpha}$ is defined as

$$
\hat{\pi}_{\alpha}=\sum_{k l} \zeta_{k l}^{\alpha} Q_{k} P_{l}
$$

$\mu_{\alpha \beta}$ is the generalized inverse inertia matrix, which can be expanded as a Taylor series in normal coordinates $[2,8,23-24]$. Since the vibrational angular momentum term occurs in the second order correction, only the leading term in the expansion, the constant $\left(I^{o^{-1}}\right)_{\alpha \beta}$, is kept. Also, the molecular axes are chosen to coincide with the principle axes of inertia so that $I_{\alpha \beta}^{o}$ vanish. Since $I^{o}$ is a diagonal matrix,

$$
\mu_{\alpha \alpha}=\frac{1}{I_{\alpha \alpha}^{o}}=\frac{2}{\hbar^{2}} B_{\alpha}
$$

where $B_{\alpha}$ is the equilibrium rotational constant about axis $\alpha$ in units of energy.

The Coriolis coupling operator can be written in terms of dimensionless normal coordinates using Equations (4) and (5) to become [24]

$$
\hat{\pi}_{\alpha}=\sum_{k l} \zeta_{k l}^{\alpha} Q_{k} P_{l}=\hbar \sum_{k l} \zeta_{k l}^{\alpha}\left(\frac{\omega_{l}}{\omega_{k}}\right)^{1 / 2} q_{k} p_{l}
$$

The most general vibrational angular momentum matrix element can be written as

$$
\left\langle a\left|\sum_{\alpha} B_{\alpha}\left(\sum_{k l} \zeta_{k l}^{\alpha}\left(\frac{\omega_{l}}{\omega_{k}}\right)^{1 / 2} q_{k} p_{l}\right)\left(\sum_{k^{\prime} l^{\prime}} \zeta_{k^{\prime} l^{\prime}}^{\alpha}\left(\frac{\omega_{l^{\prime}}}{\omega_{k^{\prime}}}\right)^{1 / 2} q_{k^{\prime}} p_{l^{\prime}}\right)\right| b\right\rangle
$$

Since the vibrational angular momentum term has been formulated, the nonzero diagonal matrix element can be found. As in IV.A.4, the numbers of distinct modes that lead to nonzero matrix elements will be identified. If four distinct modes are considered, all of the matrix element are zero, since $\left\langle\mathrm{v}_{i}\left|q_{i}\right| \mathrm{v}_{i}\right\rangle=0$ and $\left\langle\mathrm{v}_{i}\left|p_{i}\right| \mathrm{v}_{i}\right\rangle=0$. Similarly, if three distinct modes are considered, two modes will have a $q_{i}$ or $p_{i}$ with degree one, thereby 
making the entire matrix element zero. If one distinct mode is considered, then the diagonal matrix elements are zero because it would be forced to couple with itself, and $\zeta_{k k}^{\alpha}=0 \quad[2,24]$. Thus there are no nonzero diagonal terms when one, three, or four modes are involved.

However, if there are two distinct modes $k$ and $l$, then there can be nonzero diagonal matrix elements. The possible arrangements of $k$ and $l$ are

$$
q_{k} p_{k} q_{l} p_{l} \quad q_{l} p_{l} q_{k} p_{k} \quad q_{k} p_{l} q_{k} p_{l} \quad q_{l} p_{k} q_{l} p_{k} \quad q_{k} p_{l} q_{l} p_{k} \quad q_{l} p_{k} q_{k} p_{l}
$$

The arrangements $q_{k} p_{k} q_{l} p_{l}$ and $q_{l} p_{l} q_{k} p_{k}$ have matrix elements equal to zero since $\zeta_{k k}^{\alpha}=0$. Including only non-zero terms, the product of two summations given in Expression (70) can be collapsed into summations where $k \neq l$

$$
\left\langle a\left|\sum_{\alpha} B_{\alpha}\left(\sum_{k \neq l} \zeta_{k l}^{\alpha}\left(\frac{\omega_{l}}{\omega_{k}}\right)^{1 / 2} q_{k} p_{l}\right)\left(\sum_{k \neq l} \zeta_{k l}^{\alpha}\left(\frac{\omega_{l}}{\omega_{k}}\right)^{1 / 2} q_{k} p_{l}\right)\right| a\right\rangle
$$

These remaining arrangements are nonzero, and their corresponding matrix elements are

$$
\begin{aligned}
& \left\langle\mathrm{v}_{k}, \mathrm{v}_{l}\left|\zeta_{k l}^{\alpha}\left(\frac{\omega_{l}}{\omega_{k}}\right)^{1 / 2} q_{k} p_{l} \zeta_{k l}^{\alpha}\left(\frac{\omega_{l}}{\omega_{k}}\right)^{1 / 2} q_{k} p_{l}\right| \mathrm{v}_{k}, \mathrm{v}_{l}\right\rangle \\
& =\left(\zeta_{k l}^{\alpha}\right)^{2}\left(\frac{\omega_{l}}{\omega_{k}}\right)\left\langle\mathrm{v}_{k}\left|q_{k}^{2}\right| \mathrm{v}_{k}\right\rangle\left\langle\mathrm{v}_{l}\left|p_{l}^{2}\right| \mathrm{v}_{l}\right\rangle \\
& =\left(\zeta_{k l}^{\alpha}\right)^{2}\left(\frac{\omega_{l}}{\omega_{k}}\right)\left(\mathrm{v}_{k}+\frac{1}{2}\right)\left(\mathrm{v}_{l}+\frac{1}{2}\right)
\end{aligned}
$$

and

$$
\begin{aligned}
& \left\langle\mathrm{v}_{k}, \mathrm{v}_{l}\left|\zeta_{l k}^{\alpha}\left(\frac{\omega_{k}}{\omega_{l}}\right)^{1 / 2} q_{l} p_{k} \zeta_{l k}^{\alpha}\left(\frac{\omega_{k}}{\omega_{l}}\right)^{1 / 2} q_{l} p_{k}\right| \mathrm{v}_{k}, \mathrm{v}_{l}\right\rangle \\
& =\left(\zeta_{k l}^{\alpha}\right)^{2}\left(\frac{\omega_{k}}{\omega_{l}}\right)\left\langle\mathrm{v}_{l}\left|q_{l}^{2}\right| \mathrm{v}_{l}\right\rangle\left\langle\mathrm{v}_{k}\left|p_{k}^{2}\right| \mathrm{v}_{k}\right\rangle \\
& =\left(\zeta_{k l}^{\alpha}\right)^{2}\left(\frac{\omega_{k}}{\omega_{l}}\right)\left(\mathrm{v}_{l}+\frac{1}{2}\right)\left(\mathrm{v}_{k}+\frac{1}{2}\right)
\end{aligned}
$$


Since $\zeta_{k l}^{\alpha}=-\zeta_{l k}^{\alpha}$,

$$
\begin{aligned}
& \left\langle\mathrm{v}_{k}, \mathrm{v}_{l}\left|\zeta_{k l}^{\alpha}\left(\frac{\omega_{l}}{\omega_{k}}\right)^{1 / 2} q_{k} p_{l} \zeta_{l k}^{\alpha}\left(\frac{\omega_{k}}{\omega_{l}}\right)^{1 / 2} q_{l} p_{k}\right| \mathrm{v}_{k}, \mathrm{v}_{l}\right\rangle \\
& =-\left(\zeta_{k l}^{\alpha}\right)^{2}\left\langle\mathrm{v}_{k}\left|q_{k} p_{k}\right| \mathrm{v}_{k}\right\rangle\left\langle\mathrm{v}_{l}\left|p_{l} q_{l}\right| \mathrm{v}_{l}\right\rangle \\
& =-\left(\zeta_{k l}^{\alpha}\right)^{2}\left(\frac{1}{4}\right)
\end{aligned}
$$

and

$$
\begin{aligned}
& \left\langle\mathrm{v}_{k}, \mathrm{v}_{l}\left|\zeta_{l k}^{\alpha}\left(\frac{\omega_{k}}{\omega_{l}}\right)^{1 / 2} q_{l} p_{k} \zeta_{k l}^{\alpha}\left(\frac{\omega_{l}}{\omega_{k}}\right)^{1 / 2} q_{k} p_{l}\right| \mathrm{v}_{k}, \mathrm{v}_{l}\right\rangle \\
& =-\left(\zeta_{k l}^{\alpha}\right)^{2}\left\langle\mathrm{v}_{k}\left|p_{k} q_{k}\right| \mathrm{v}_{k}\right\rangle\left\langle\mathrm{v}_{l}\left|q_{l} p_{l}\right| \mathrm{v}_{l}\right\rangle \\
& =-\left(\zeta_{k l}^{\alpha}\right)^{2}\left(\frac{1}{4}\right)
\end{aligned}
$$

The last two matrix element types are constants and will be included in the zero-point energy. Thus, only the arrangements $q_{k} p_{l} q_{k} p_{l}$ and $q_{l} p_{k} q_{l} p_{k}$ lead to nonzero, $\mathrm{v}_{\mathrm{i}}$-dependent diagonal matrix elements.

To further emphasize that modes $k$ and $l$ from the second summation must equal the modes $k$ or $l$ in the first summation, Expression (70) can be expressed more compactly in terms of these matrix element types as

$$
\begin{aligned}
& \left\langle a\left|\sum_{\alpha} B_{\alpha}\left(\sum_{k \neq l} \zeta_{k l}^{\alpha}\left(\frac{\omega_{l}}{\omega_{k}}\right)^{1 / 2} q_{k} p_{l}\right)\left(\sum_{k \neq l} \zeta_{k l}^{\alpha}\left(\frac{\omega_{l}}{\omega_{k}}\right)^{1 / 2} q_{k} p_{l}\right)\right| a\right\rangle \\
& =\left\langle a\left|\sum_{\alpha} B_{\alpha}\left(\sum_{k \neq l} \zeta_{k l}^{\alpha}\left(\frac{\omega_{l}}{\omega_{k}}\right)^{1 / 2} q_{k} p_{l}\right)\left(\zeta_{k l}^{\alpha}\left(\frac{\omega_{l}}{\omega_{k}}\right)^{1 / 2} q_{k} p_{l}+\zeta_{l k}^{\alpha}\left(\frac{\omega_{k}}{\omega_{l}}\right)^{1 / 2} q_{l} p_{k}\right)\right| a\right\rangle \\
& =\left\langle a\left|\sum_{\alpha} B_{\alpha} \sum_{k \neq l}\left(\zeta_{k l}^{\alpha} \zeta_{k l}^{\alpha}\left(\frac{\omega_{l}}{\omega_{k}}\right)^{1 / 2}\left(\frac{\omega_{l}}{\omega_{k}}\right)^{1 / 2} q_{k} p_{l} q_{k} p_{l}\right)+\sum_{\alpha} B_{\alpha} \sum_{k \neq l}\left(\zeta_{k l}^{\alpha} \zeta_{l k}^{\alpha}\left(\frac{\omega_{l}}{\omega_{k}}\right)^{1 / 2}\left(\frac{\omega_{k}}{\omega_{l}}\right)^{1 / 2} q_{k} p_{l} q_{l} p_{k}\right)\right| a\right\rangle
\end{aligned}
$$

Since the two parts of Equation (72) involve the multiplication of summations, terms within a summation are multiplied by terms in the other summation. Thus, as shown above, all of the 
four arrangements of modes $k$ and $l$ will occur. The first term in Equation (72) gives the nonzero, $\mathrm{v}_{\mathrm{i}}$-dependent part of the vibrational angular momentum contribution, and the second gives the constant vibrational angular momentum contribution to the zero-point energy.

After evaluating the matrix elements, Equation (72) becomes

$$
\begin{aligned}
& \left\langle a\left|\sum_{\alpha} B_{\alpha} \sum_{k \neq l}\left(\zeta_{k l}^{\alpha} \zeta_{k l}^{\alpha}\left(\frac{\omega_{l}}{\omega_{k}}\right)^{1 / 2}\left(\frac{\omega_{l}}{\omega_{k}}\right)^{1 / 2} q_{k} p_{l} q_{k} p_{l}\right)+\sum_{\alpha} B_{\alpha} \sum_{k \neq l}\left(\zeta_{k l}^{\alpha} \zeta_{l k}^{\alpha}\left(\frac{\omega_{l}}{\omega_{k}}\right)^{1 / 2}\left(\frac{\omega_{k}}{\omega_{l}}\right)^{1 / 2} q_{k} p_{l} q_{l} p_{k}\right)\right| a\right\rangle \\
& =\sum_{\alpha} B_{\alpha} \sum_{k \neq l}\left(\zeta_{k l}^{\alpha}\right)^{2}\left(\frac{\omega_{l}}{\omega_{k}}\right)\left(\mathrm{v}_{k}+\frac{1}{2}\right)\left(\mathrm{v}_{l}+\frac{1}{2}\right)+\sum_{\alpha} B_{\alpha} \sum_{k \neq l}\left(\zeta_{k l}^{\alpha}\right)^{2}\left(-\frac{1}{4}\right)
\end{aligned}
$$

Given the results of sections IV.A.2-IV.A.5, the on-diagonal matrix elements are

$$
\begin{aligned}
& \langle a|\tilde{H}| a\rangle=\tilde{H}_{a}^{o}+\tilde{H}_{a a}^{\prime}+\tilde{H}_{a a}^{\prime \prime}=H_{a}^{o}+0+H_{a a}^{\prime \prime}+\sum_{\gamma \neq a} \frac{H_{a \gamma}^{\prime} H_{\gamma a}^{\prime}}{E_{a}^{o}-E_{\gamma}^{o}} \\
& =h c \sum_{k} \omega_{k}\left(\mathrm{v}_{k}+\frac{1}{2}\right)+h c \frac{1}{16} \sum_{k} \phi_{k k k k}\left[\left(\mathrm{v}_{k}+\frac{1}{2}\right)^{2}+\frac{1}{4}\right]+\frac{1}{8} h c \sum_{k \neq l} \phi_{k k l l}\left(\mathrm{v}_{k}+\frac{1}{2}\right)\left(\mathrm{v}_{l}+\frac{1}{2}\right) \\
& +\sum_{\alpha} B_{\alpha} \sum_{k \neq l}\left(\zeta_{k l}^{\alpha}\right)^{2}\left[\left(\frac{\omega_{l}}{\omega_{k}}\right)\left(\mathrm{v}_{k}+\frac{1}{2}\right)\left(\mathrm{v}_{l}+\frac{1}{2}\right)-\frac{1}{4}\right]-h c \sum_{k \neq l} \frac{1}{16} \phi_{k k l}^{2} \frac{\left(8 \omega_{k}^{2}-3 \omega_{l}^{2}\right)}{\omega_{l}\left(4 \omega_{k}^{2}-\omega_{l}^{2}\right)}\left(\mathrm{v}_{k}+\frac{1}{2}\right)^{2} \\
& -h c \sum_{k} \frac{5}{48} \phi_{k k k}^{2} \frac{1}{\omega_{k}}\left(\mathrm{v}_{k}+\frac{1}{2}\right)^{2}-h c \sum_{k \neq l \neq m} \frac{1}{8} \phi_{k k m} \phi_{l l m} \frac{1}{\omega_{m}}\left(\mathrm{v}_{k}+\frac{1}{2}\right)\left(\mathrm{v}_{l}+\frac{1}{2}\right) \\
& -h c \sum_{k \neq l} \frac{1}{4} \phi_{k k k} \phi_{k l l} \frac{1}{\omega_{k}}\left(\mathrm{v}_{k}+\frac{1}{2}\right)\left(\mathrm{v}_{l}+\frac{1}{2}\right)-h c \sum_{k \neq l \neq m} \frac{\phi_{k l m}^{2}}{12 N_{k l m}} \omega_{m}\left(\omega_{m}^{2}-\omega_{l}^{2}-\omega_{k}^{2}\right)\left(\mathrm{v}_{k}+\frac{1}{2}\right)\left(\mathrm{v}_{l}+\frac{1}{2}\right) \\
& -h c \sum_{k \neq l \neq m} \frac{\phi_{k l m}^{2}}{12 N_{k l m}} \omega_{l}\left(\omega_{l}^{2}-\omega_{k}^{2}-\omega_{m}^{2}\right)\left(\mathrm{v}_{k}+\frac{1}{2}\right)\left(\mathrm{v}_{m}+\frac{1}{2}\right) \\
& -h c \sum_{k \neq l \neq m} \frac{\phi_{k l m}^{2}}{12 N_{k l m}} \omega_{k}\left(\omega_{k}^{2}-\omega_{l}^{2}-\omega_{m}^{2}\right)\left(\mathrm{v}_{l}+\frac{1}{2}\right)\left(\mathrm{v}_{m}+\frac{1}{2}\right)-h c \sum_{k \neq l} \frac{\phi_{k k l}^{2}}{2} \frac{\omega_{k}}{\left(4 \omega_{k}^{2}-\omega_{l}^{2}\right)}\left(\mathrm{v}_{k}+\frac{1}{2}\right)\left(\mathrm{v}_{l}+\frac{1}{2}\right) \\
& -h c \sum_{k \neq l \neq m} \frac{\phi_{k l m}^{2}}{24 N_{k l m}} \omega_{k} \omega_{l} \omega_{m}+h c \sum_{k \neq l} \frac{3}{64} \phi_{k k l}^{2} \frac{\omega_{l}}{\left(4 \omega_{k}^{2}-\omega_{l}^{2}\right)}-h c \sum_{k} \frac{7}{576} \phi_{k k k}^{2}
\end{aligned}
$$

\section{Spectroscopic constants}

To condense Equation (74) even further, the anharmonicity coefficients $x_{k k}$ and $x_{k l}$ will be derived. These coefficients are important spectroscopic constants that can be determined from experimental data. 
From Equation (22), the energy correction can be written as

$$
E=E^{o}+E^{\prime}+E^{\prime \prime}+\ldots
$$

when the arbitrary ordering parameter $\lambda$ equals 1 . In Equation (75), the first term is the zeroorder energy and the primed terms represent the first- and second-order energy corrections, respectively. As stated previously, only terms up to second order will be considered.

The energy also can be written in terms of quantum numbers, as a Dunham expansion of increasing powers of $v_{i}[1,25]$

$$
E=h c \sum_{k} \omega_{k}\left(\mathrm{v}_{k}+\frac{1}{2}\right)+\sum_{k \geq l} x_{k l}\left(\mathrm{v}_{k}+\frac{1}{2}\right)\left(\mathrm{v}_{l}+\frac{1}{2}\right)+\ldots
$$

The constants $x_{k k}$ and $x_{k l}$ are the anharmonicity coefficients, and they are derived from the restrictive counterpart of Equation (74) by grouping terms with $\left(\mathrm{v}_{k}+\frac{1}{2}\right)^{2}$ and $\left(\mathrm{v}_{k}+\frac{1}{2}\right)\left(\mathrm{v}_{l}+\frac{1}{2}\right)$, respectively. The constant $x_{k k}$ occurs when $k=l$, and $x_{k l}$ occurs when $k \neq l$. Note that $x_{k k}$ and $x_{k l}$ do not include the constant terms from Equation (74) not involving quantum numbers, which are incorporated into the zero-point energy and therefore neglected in the final $x_{k k}$ and $x_{k l}$ expressions. The ignored constant terms are

$$
C=h c \sum_{k} \frac{1}{64} \phi_{k k k k}-h c \sum_{k \neq l \neq m} \frac{\phi_{k l m}^{2}}{24 N_{k l m}} \omega_{k} \omega_{l} \omega_{m}+h c \sum_{k \neq l} \frac{3}{64} \phi_{k k l}^{2} \frac{\omega_{l}}{\left(4 \omega_{k}^{2}-\omega_{l}^{2}\right)}-h c \sum_{k} \frac{7}{576} \phi_{k k k}^{2}-\frac{1}{4} \sum_{\alpha} B_{\alpha} \sum_{k \neq l}\left(\zeta_{k l}^{\alpha}\right)^{2}
$$

Though the derivations thus far have been non-restrictive, the anharmonicity coefficients are traditionally written as restrictive expressions. These results are presented using the restricted sums, since the anharmonicity coefficients are inherently restrictive quantities. The restriction $k \geq l$ has been imposed on the second summation in Equation (76) so that only one cross term of each type is evaluated. That is, in converting from a non-restrictive summation to a 
restrictive one, the two non-restrictive terms $x_{k l}$ and $x_{l k}$ are condensed into one restrictive term so that $x_{k l}$ is equal to their sum.

The anharmonicity coefficient $x_{k k}$ occurs when $k=l$, and so $x_{k k}$ is a collection of the restrictive counterparts of the $\left(\mathrm{v}_{k}+\frac{1}{2}\right)^{2}$ terms in Equation (74). There is no vibrational angular momentum term in $x_{k k}$ because a mode cannot couple with itself. Then the anharmonic constant $x_{k k}$ becomes

$$
x_{k k}=h c \frac{1}{16} \phi_{k k k k}-h c \sum_{l} \frac{1}{16} \phi_{k k l}^{2} \frac{\left(8 \omega_{k}^{2}-3 \omega_{l}^{2}\right)}{16 \omega_{l}\left(4 \omega_{k}^{2}-\omega_{l}^{2}\right)}
$$

The anharmonicity coefficient $x_{k l}$ occurs when $k \neq l$, and so $x_{k l}$ is a collection of the $\left(\mathrm{v}_{k}+\frac{1}{2}\right)\left(\mathrm{v}_{l}+\frac{1}{2}\right)$ terms. There is a vibrational angular momentum contribution to $\mathrm{x}_{\mathrm{kl}}$ because Equation (74) contains a vibrational angular momentum term with the quantum number dependence $\left(\mathrm{v}_{k}+\frac{1}{2}\right)\left(\mathrm{v}_{l}+\frac{1}{2}\right)$. Then the expression for $x_{k l}$ is $x_{k l}=h c \frac{1}{4} \phi_{k k l l}-h c \sum_{m} \frac{\phi_{k l m}^{2}}{2 N_{k l m}} \omega_{m}\left(\omega_{m}^{2}-\omega_{l}^{2}-\omega_{k}^{2}\right)-h c \sum_{m} \phi_{k k m} \phi_{l l m} \frac{1}{4 \omega_{m}}+\sum_{\alpha} B_{\alpha}\left(\xi_{k l}^{\alpha}\right)^{2}\left(\frac{\omega_{l}}{\omega_{k}}+\frac{\omega_{k}}{\omega_{l}}\right)$

where $N_{k l m}$ is defined in Equation (64). To match Papoušek and Aliev's convention [2] for $x_{k l}$, a factor of -1 must be exchanged between the numerator and denominator of the second term in Equation (79), yielding the final $x_{k l}$ expression

$$
x_{k l}=h c \frac{1}{4} \phi_{k k l l}-h c \sum_{m} \frac{\phi_{k l m}^{2}}{2 \Omega_{k l m}} \omega_{m}\left(\omega_{k}^{2}+\omega_{l}^{2}-\omega_{m}^{2}\right)-h c \sum_{m} \phi_{k k m} \phi_{l l m} \frac{1}{4 \omega_{m}}+\sum_{\alpha} B_{\alpha}\left(\zeta_{k l}^{\alpha}\right)^{2}\left(\frac{\omega_{l}}{\omega_{k}}+\frac{\omega_{k}}{\omega_{l}}\right)
$$

where

$$
\Omega_{k l m}=-N_{k l m}=\left(\omega_{k}+\omega_{l}+\omega_{m}\right)\left(\omega_{k}+\omega_{l}-\omega_{m}\right)\left(\omega_{k}-\omega_{l}+\omega_{m}\right)\left(-\omega_{k}+\omega_{l}+\omega_{m}\right)
$$




\section{B. Off-diagonal matrix elements}

\section{Types of resonances}

Van Vleck perturbation theory (VVPT) is capable of treating strongly interacting states in resonance. Their interactions are made manifest in distinct types of off-diagonal matrix elements among class 1 states. Resonances can connect several vibrational levels. Furthermore, multiple resonances can connect a network of levels, which are called a polyad [20, 26-27]. Second order perturbation theory (PT2) does not treat resonances, as it allows for just a single class 1 state to interact with all other class 2 states. Off-diagonal matrix elements of the vibrational Hamiltonian are evaluated using VVPT by a process of classifying nonzero matrix elements, performing algebraic manipulations, and regrouping summations similar to the process performed in IV.A. The off-diagonal elements will be presented according to the specific resonance that gives rise to the large interaction.

Recall that the effective Hamiltonian can be expanded according to Equations (46)(49). There are no nonzero zeroth order off-diagonal terms since $\widetilde{H}^{o}$ is a diagonal matrix for both class 1 and class 2 states. The presence of an off-diagonal first order correction $\widetilde{H}^{\prime}$ depends on the existence of Fermi resonances. Many types of resonances lead to offdiagonal second order energy corrections $\widetilde{H}^{\prime \prime}$. The majority of the second-order corrections are small, though resonances give rise to some large terms. In the case of resonances, the offdiagonal matrix elements must be treated explicitly, e.g., by excluding class 1 states from the sum over $\gamma$ in Equation (49) or, as will be demonstrated in V.C., by removing terms with the appropriate resonance denominators.

Resonances can be classified according to the total change of quanta. For a Fermi resonance, a total of three quanta change, where one vibrational frequency is approximately the same as the sum of two other vibrational frequencies (1-2 resonance). There are multiple 
resonances for which four quanta change. For a Darling-Dennison resonance, the sum of two vibrational frequencies are approximately equal to the sum of two other vibrational frequencies (2-2 resonance). Also, there are second order resonances in which one vibrational frequency is approximately equal to the sum of three other vibrational frequencies (1-3 resonance). Another type of resonance involves a total change of two quanta, with two vibrational frequencies that are approximately equal (1-1 resonance). The 1-1 resonance is actually an extension of multiple 2-2 resonances with a common mode between the two states involved in the resonance.

\section{1-2 resonances}

A 1-2 resonance, or Fermi resonance, involves the annihilation of one quantum in one mode and the creation of two quanta in one or two other modes. The matrix element of a Fermi resonance involving three distinct modes is

$$
\left\langle\ldots n_{k}+1, n_{l}, n_{m} \ldots|\tilde{H}| \ldots n_{k}, n_{l}+1, n_{m}+1 \ldots\right\rangle
$$

Equations (47)-(49) will be used to evaluate the above matrix element. Since $\widetilde{H}^{o}$ is purely diagonal, there is no off-diagonal element due to $\widetilde{H}^{o}$. That is,

$$
\left\langle\ldots n_{k}+1, n_{l}, n_{m} \ldots\left|\widetilde{H}^{o}\right| \ldots n_{k}, n_{l}+1, n_{m}+1 \ldots\right\rangle=0
$$

However, the first-order energy correction $\widetilde{H}^{\prime}$ is

$$
\begin{aligned}
& \left\langle\ldots n_{k}+1, n_{l}, n_{m} \ldots\left|\widetilde{H}^{\prime}\right| \ldots n_{k}, n_{l}+1, n_{m}+1 \ldots\right\rangle \\
= & \left\langle\ldots n_{k}+1, n_{l}, n_{m} \ldots\left|H^{\prime}\right| \ldots n_{k}, n_{l}+1, n_{m}+1 \ldots\right\rangle \\
= & \left\langle\ldots n_{k}+1, n_{l}, n_{m} \ldots\left|h c \sum_{k l m} \frac{1}{3 !} \phi_{k l m} q_{k} q_{l} q_{m}\right| \ldots n_{k}, n_{l}+1, n_{m}+1 \ldots\right\rangle \\
= & h c \frac{3 !}{1 ! 1 ! !} \cdot \frac{1}{3 !} \phi_{k l m} \frac{\left(n_{k}+1\right)^{1 / 2}\left(n_{l}+1\right)^{1 / 2}\left(n_{m}+1\right)^{1 / 2}}{2^{3 / 2}} \\
= & h c \phi_{k l m} \frac{\left(n_{k}+1\right)^{1 / 2}\left(n_{l}+1\right)^{1 / 2}\left(n_{m}+1\right)^{1 / 2}}{2^{3 / 2}}
\end{aligned}
$$


In Equation (83), the sum over mode indices $k l m$ evaluates the six permutations of $\phi_{k l m}$. The permutation factor of $\frac{3 !}{1 ! 1 ! !}$ is included to account for all of these permutations.

The second order energy correction is zero. The quartic part of Equation (49) is zero because a maximum of only three modes change in the matrix element, not four, and $\left\langle\mathrm{v}_{i}\left|q_{i}\right| \mathrm{v}_{i}\right\rangle=0$ for the fourth mode. The vibrational angular momentum part is also zero. When four distinct modes are considered, a non-resonant mode $n$ has a $p$ or $q$ operator of degree one, which yields a matrix element of zero. For three distinct modes, one of the resonant modes has a total degree of two for its $p$ and $q$ operators, which evaluates to a matrix element of zero for a change of one quantum. If only one or two distinct modes are considered, the matrix element for the other resonant mode(s) is zero. The second order cubic part of Equation (49) is zero because there is no intermediate state $\gamma$ that leads to nonzero matrix elements in which the overall change in quanta in each bra-ket is at most three quanta. There are six $q_{i}$ total, and three $q_{i}$ must be used for modes $k, l$, and $m$ involved in the resonance. Matrix elements with the corresponding force constants of the form $\phi_{k l m} \phi_{r r r}$ or $\phi_{k l r} \phi_{m r r}$ result, where $r$ is an arbitrary mode that may or may not equal $k, l$, or $m$. If $r \neq k, l$, or $m$, the overall degree of $q_{r}$ is three, which cannot connect states with no change in quanta in mode $r$. If $r=k, l$, or $m$, non-zero matrix elements would involve some mode $k, l$, or $m$ changing by zero, two, or four quanta, which would not connect to the appropriate resonant state.

Thus

$$
\left\langle\ldots n_{k}+1, n_{l}, n_{m} \ldots\left|\tilde{H}^{\prime \prime}\right| \ldots n_{k}, n_{l}+1, n_{m}+1 \ldots\right\rangle=0
$$

Hence, the overall off-diagonal matrix element for the most general 1-2 resonance is 


$$
\left\langle\ldots n_{k}+1, n_{l}, n_{m} \ldots|\tilde{H}| \ldots n_{k}, n_{l}+1, n_{m}+1 \ldots\right\rangle=K_{k ; l m} \frac{\left(n_{k}+1\right)^{1 / 2}\left(n_{l}+1\right)^{1 / 2}\left(n_{m}+1\right)^{1 / 2}}{2^{3 / 2}}
$$

where

$$
K_{k ; l m}=h c \phi_{k l m}
$$

Another type of Fermi resonance involves the annihilation of a quantum in one mode and the creation of two quanta in another mode. Its associated matrix element is

$$
\left\langle\ldots n_{k}+1, n_{l} \ldots|\tilde{H}| \ldots n_{k}, n_{l}+2 \ldots\right\rangle
$$

The matrix element for this 1-2 resonance can be derived in the manner presented above for the most general Fermi resonance. The zeroth and second order corrections are zero for the same reasons stated above

$$
\begin{aligned}
& \left\langle\ldots n_{k}+1, n_{l} \ldots\left|\tilde{H}^{o}\right| \ldots n_{k}, n_{l}+2 \ldots\right\rangle=0 \\
& \left\langle\ldots n_{k}+1, n_{l} \ldots\left|\tilde{H}^{\prime \prime}\right| \ldots n_{k}, n_{l}+2 \ldots\right\rangle=0
\end{aligned}
$$

Only the first order correction is nonzero

$$
\begin{aligned}
& \left\langle\ldots n_{k}+1, n_{l} \ldots\left|\tilde{H}^{\prime}\right| \ldots n_{k}, n_{l}+2 \ldots\right\rangle \\
= & \left\langle\ldots n_{k}+1, n_{l} \ldots\left|H^{\prime}\right| \ldots n_{k}, n_{l}+2 \ldots\right\rangle \\
= & \left\langle\ldots n_{k}+1, n_{l} \ldots\left|h c \sum_{k l m} \frac{1}{3 !} \phi_{k l m} q_{k} q_{l} q_{m}\right| \ldots n_{k}, n_{l}+2 \ldots\right\rangle \\
= & \left\langle\ldots n_{k}+1, n_{l} \ldots\left|h c \frac{3 !}{1 ! 2 !} \cdot \frac{1}{3 !} \phi_{k l l} q_{k} q_{l}^{2}\right| \ldots n_{k}, n_{l}+2 \ldots\right\rangle \\
= & h c \frac{3 !}{1 ! 2 !} \cdot \frac{1}{3 !} \phi_{k l l} \cdot \frac{\left(n_{k}+1\right)^{1 / 2}}{2^{1 / 2}} \cdot \frac{\left(n_{l}+1\right)^{1 / 2}\left(n_{l}+2\right)^{1 / 2}}{2} \\
= & h c \frac{\phi_{k l l}}{2} \frac{\left(n_{k}+1\right)^{1 / 2}\left(n_{l}+1\right)^{1 / 2}\left(n_{l}+2\right)^{1 / 2}}{2^{3 / 2}}
\end{aligned}
$$

Thus, the off-diagonal matrix element for this Fermi resonance is

$$
\left\langle\ldots n_{k}+1, n_{l} \ldots|\tilde{H}| \ldots n_{k}, n_{l}+2 \ldots\right\rangle=K_{k ; l l} \frac{\left(n_{k}+1\right)^{1 / 2}\left(n_{l}+1\right)^{1 / 2}\left(n_{l}+2\right)^{1 / 2}}{2^{3 / 2}}
$$


where

$$
K_{k ; l l}=h c \frac{\phi_{k l l}}{2}
$$

Note that the resonance constant for the second type of Fermi resonance in Equation

(89) differs from the first type given in Equation (83) by a constant, namely the factor of $\frac{3 !}{1 ! 2 !}$.

This is a result of the permutation factor associated with the $\phi_{k l l}$ force constant.

\section{2-2 resonances}

a. General 2-2 resonance. The most general 2-2 resonance involves the annihilation of two quanta in two modes and the creation of two quanta in two different modes. The most general matrix element of a 2-2 resonance is

$$
\left\langle n_{k}+1, n_{l}+1, n_{m}, n_{n}|\widetilde{H}| n_{k}, n_{l}, n_{m}+1, n_{n}+1\right\rangle
$$

where $k \neq l \neq m \neq n$.

As with the 1-2 resonances, the zeroth-order energy contribution is zero

$$
\left\langle n_{k}+1, n_{l}+1, n_{m}, n_{n}\left|\tilde{H}^{o}\right| n_{k}, n_{l}, n_{m}+1, n_{n}+1\right\rangle=0
$$

The first-order contribution is also zero. In all 2-2 resonances, there is a total change of four quanta. In Equation (48), a change of one or three quanta is permitted. Thus

$$
\left\langle n_{k}+1, n_{l}+1, n_{m}, n_{n}\left|\tilde{H}^{\prime}\right| n_{k}, n_{l}, n_{m}+1, n_{n}+1\right\rangle=0
$$

The second-order off-diagonal matrix element consists of three parts, the quartic, vibrational angular momentum, and cubic terms. Each part will be derived separately.

The quartic contribution to $\widetilde{H}^{\prime \prime}$ is the simplest to evaluate using Table 1 


$$
\begin{aligned}
& \left\langle n_{k}+1, n_{l}+1, n_{m}, n_{n}\left|h c \sum_{k l m n} \frac{1}{4 !} \phi_{k l m n} q_{k} q_{l} q_{m} q_{n}\right| n_{k}, n_{l}, n_{m}+1, n_{n}+1\right\rangle \\
& =h c \frac{4 !}{1 ! 1 ! 1 ! ! !} \cdot \frac{1}{4 !} \phi_{k l m n} \frac{1}{4}\left[\left(n_{k}+1\right)\left(n_{l}+1\right)\left(n_{m}+1\right)\left(n_{n}+1\right)\right]^{1 / 2} \\
& =h c \phi_{k l m n} \frac{1}{4}\left[\left(n_{k}+1\right)\left(n_{l}+1\right)\left(n_{m}+1\right)\left(n_{n}+1\right)\right]^{1 / 2}
\end{aligned}
$$

The vibrational angular momentum contributions to $\widetilde{H}^{\prime \prime}$ are more complicated to formulate. Recall from IV.A.5 that this term appears as

$$
\left\langle a \mid \sum_{\alpha} B_{\alpha}\left(\sum_{k l} \zeta_{k l}^{\alpha}\left(\frac{\omega_{l}}{\omega_{k}}\right)^{1 / 2} q_{k} p_{l}\right)\left(\sum_{k^{\prime} l^{\prime}} \zeta_{k^{\prime} l^{\prime}}^{\alpha}\left(\frac{\omega_{l^{\prime}}}{\omega_{k^{\prime}}}\right)^{1 / 2} q_{k^{\prime}} p_{l^{\prime}}\right) b b\right\rangle
$$

In the case of the most general 2-2 resonance, only terms that incorporate four distinct modes are nonzero, i.e., where $k \neq l \neq m \neq n$, because terms involving three or less modes cannot yield the appropriate matrix element, e.g., $\left\langle n_{k}+1, n_{l}+1, n_{m}, n_{n}\left|q_{k} p_{l} q_{m} p_{n}\right| n_{k}, n_{l}, n_{m}+1, n_{n}+1\right\rangle$, and so have zero contribution, and also since they would include $\zeta_{i i}^{\alpha}$, which is zero.

Since there are four unique modes in the most general 2-2 resonance, there are $4 !=24$ ways to arrange four modes where order matters. The twenty-four arrangements can be enumerated by listing all possible arrangements of four distinct objects. Consider four distinct modes, $k, l, m, n$, involved in a 2-2 resonance. Then the twenty-four possible arrangements of operators are

$$
\begin{array}{cccc}
q_{k} p_{l} q_{m} p_{n} & q_{l} p_{k} q_{m} p_{n} & q_{k} p_{m} q_{l} p_{n} & q_{m} p_{k} q_{l} p_{n} \\
q_{k} p_{l} q_{n} p_{m} & q_{l} p_{k} q_{n} p_{m} & q_{k} p_{m} q_{n} p_{l} & q_{m} p_{k} q_{n} p_{l} \\
q_{n} p_{k} q_{l} p_{m} & q_{k} p_{n} q_{l} p_{m} & q_{n} p_{k} q_{m} p_{l} & q_{k} p_{n} q_{m} p_{l} \\
q_{l} p_{m} q_{k} p_{n} & q_{m} p_{l} q_{k} p_{n} & q_{l} p_{m} q_{n} p_{k} & q_{m} p_{l} q_{n} p_{k} \\
q_{l} p_{n} q_{k} p_{m} & q_{n} p_{l} q_{k} p_{m} & q_{m} p_{n} q_{k} p_{l} & q_{n} p_{m} q_{k} p_{l} \\
q_{l} p_{n} q_{m} p_{k} & q_{n} p_{l} q_{m} p_{k} & q_{m} p_{n} q_{l} p_{k} & q_{n} p_{m} q_{l} p_{k}
\end{array}
$$


It is important to notice that the order within a pair matters, $e . g ., q_{k} p_{l}$ is different than $q_{l} p_{k}$. Since the multiplication of two Coriolis coupling operators involves distinct modes, the order of pairs does not matter $\left(e . g ., q_{k} p_{l} q_{m} p_{n}=q_{m} p_{n} q_{k} p_{l}\right)$. Consequently, there are only twelve unique terms, but each term appears twice.

The matrix elements and Coriolis coupling constants for the top-half of the above arrangements are listed in the supplemental material. Some of the matrix elements have been rewritten using the relation $\zeta_{k l}^{\alpha}=-\zeta_{l k}^{\alpha}$ so that each term has Coriolis constants $\zeta_{k l}^{\alpha} \zeta_{m n}^{\alpha}$, $\zeta_{k m}^{\alpha} \zeta_{l n}^{\alpha}$, or $\zeta_{k n}^{\alpha} \zeta_{l m}^{\alpha}$. Note that although the $p_{i}$ operators are complex, the imaginary parts multiply together to give real matrix elements, and the overall matrix elements are identical to those produced by normal coordinate operators $q_{i}$. By combining the terms according to their Coriolis coupling constants, the vibrational angular momentum term can be formulated. The remaining twelve arrangements not included in the supplemental material are accounted for by the factor of two that is incorporated into the expression.

$$
\begin{aligned}
& \left\langle n_{k}+1, n_{l}+1, n_{m}, n_{n} \mid \sum_{\alpha} B_{\alpha}\left(\sum_{k l} \zeta_{k l}^{\alpha}\left(\frac{\omega_{l}}{\omega_{k}}\right)^{1 / 2} q_{k} p_{l}\right)\left(\sum_{k^{\prime} l^{\prime}} \zeta_{k^{\prime} l^{\prime}}^{\alpha}\left(\frac{\omega_{l^{\prime}}}{\omega_{k^{\prime}}}\right)^{1 / 2} q_{k^{\prime}} p_{l^{\prime}}\right) n_{k}, n_{l}, n_{m}+1, n_{n}+1\right\rangle \\
& =\frac{1}{4}\left[\left(n_{k}+1\right)\left(n_{l}+1\right)\left(n_{m}+1\right)\left(n_{n}+1\right)\right]^{1 / 2} \cdot \\
& 2 \sum_{\alpha} B_{\alpha} \zeta_{k l}^{\alpha} \zeta_{m n}^{\alpha}\left\{\left[\left(\frac{\omega_{l}}{\omega_{k}} \cdot \frac{\omega_{n}}{\omega_{m}}\right)^{1 / 2}+\left(\frac{\omega_{k}}{\omega_{l}} \cdot \frac{\omega_{m}}{\omega_{n}}\right)^{1 / 2}-\left(\frac{\omega_{l}}{\omega_{k}} \cdot \frac{\omega_{m}}{\omega_{n}}\right)^{1 / 2}-\left(\frac{\omega_{k}}{\omega_{l}} \cdot \frac{\omega_{n}}{\omega_{m}}\right)^{1 / 2}\right]\right. \\
& +\zeta_{k m}^{\alpha} \zeta_{l n}^{\alpha}\left[-\left(\frac{\omega_{m}}{\omega_{k}} \cdot \frac{\omega_{l}}{\omega_{n}}\right)^{1 / 2}-\left(\frac{\omega_{n}}{\omega_{l}} \cdot \frac{\omega_{k}}{\omega_{m}}\right)^{1 / 2}-\left(\frac{\omega_{m}}{\omega_{k}} \cdot \frac{\omega_{n}}{\omega_{l}}\right)^{1 / 2}-\left(\frac{\omega_{k}}{\omega_{m}} \cdot \frac{\omega_{l}}{\omega_{n}}\right)^{1 / 2}\right]
\end{aligned}
$$




$$
\begin{aligned}
& \left.+\zeta_{k n}^{\alpha} \zeta_{l m}^{\alpha}\left[-\left(\frac{\omega_{n}}{\omega_{k}} \cdot \frac{\omega_{l}}{\omega_{m}}\right)^{1 / 2}-\left(\frac{\omega_{k}}{\omega_{n}} \cdot \frac{\omega_{m}}{\omega_{l}}\right)^{1 / 2}-\left(\frac{\omega_{n}}{\omega_{k}} \cdot \frac{\omega_{m}}{\omega_{l}}\right)^{1 / 2}-\left(\frac{\omega_{l}}{\omega_{m}} \cdot \frac{\omega_{k}}{\omega_{n}}\right)^{1 / 2}\right]\right\} \\
& =\frac{1}{4}\left[\left(n_{k}+1\right)\left(n_{l}+1\right)\left(n_{m}+1\right)\left(n_{n}+1\right)\right]^{1 / 2} \cdot 2 \sum_{\alpha} B_{\alpha}\left[\frac{\zeta_{k l}^{\alpha} \zeta_{m n}^{\alpha}\left(\omega_{k} \omega_{m}-\omega_{k} \omega_{n}-\omega_{l} \omega_{m}+\omega_{l} \omega_{n}\right)}{\left(\omega_{k} \omega_{l} \omega_{m} \omega_{n}\right)^{1 / 2}}\right. \\
& \left.-\frac{\zeta_{k m}^{\alpha} \zeta_{l n}^{\alpha}\left(\omega_{k} \omega_{l}+\omega_{k} \omega_{n}+\omega_{m} \omega_{l}+\omega_{m} \omega_{n}\right)}{\left(\omega_{k} \omega_{l} \omega_{m} \omega_{n}\right)^{1 / 2}}-\frac{\zeta_{k n}^{\alpha} \zeta_{l m}^{\alpha}\left(\omega_{m} \omega_{n}+\omega_{l} \omega_{k}+\omega_{l} \omega_{n}+\omega_{k} \omega_{m}\right)}{\left(\omega_{k} \omega_{l} \omega_{m} \omega_{n}\right)^{1 / 2}}\right] \\
& =\frac{1}{4}\left[\left(n_{k}+1\right)\left(n_{l}+1\right)\left(n_{m}+1\right)\left(n_{n}+1\right)\right]^{1 / 2} \text {. } \\
& 2 \sum_{\alpha} B_{\alpha}\left[\frac{\zeta_{k l}^{\alpha} \zeta_{m n}^{\alpha}\left(\omega_{k}-\omega_{l}\right)\left(\omega_{m}-\omega_{n}\right)-\zeta_{k m}^{\alpha} \zeta_{l n}^{\alpha}\left(\omega_{k}+\omega_{m}\right)\left(\omega_{l}+\omega_{n}\right)-\zeta_{k n}^{\alpha} \zeta_{l m}^{\alpha}\left(\omega_{k}+\omega_{n}\right)\left(\omega_{l}+\omega_{m}\right)}{\left(\omega_{k} \omega_{l} \omega_{m} \omega_{n}\right)^{1 / 2}}\right]
\end{aligned}
$$

Lastly, the cubic potential term's contribution to $\widetilde{H}^{\prime \prime}$ will be derived. The cubic terms in the effective vibrational Hamiltonian have the form of the last term in Equation (49)

$$
\begin{aligned}
& \frac{1}{2} \sum_{\gamma}\left(\frac{H_{a \gamma}^{\prime} H_{\gamma b}^{\prime}}{E_{a}^{o}-E_{\gamma}^{o}}+\frac{H_{a \gamma}^{\prime} H_{\gamma b}^{\prime}}{E_{b}^{o}-E_{\gamma}^{o}}\right) \\
& =\frac{1}{2} \sum_{\gamma}\left\langle a\left|h c \sum_{k l m} \frac{1}{3 !} \phi_{k l m} q_{k} q_{l} q_{m}\right| \gamma\right\rangle\left\langle\gamma\left|h c \sum_{k^{\prime} l^{\prime} m^{\prime}} \frac{1}{3 !} \phi_{k^{\prime} l^{\prime} m^{\prime}} q_{k^{\prime}} q_{l^{\prime}} q_{m^{\prime}}\right| b\right\rangle\left(\frac{1}{E_{a}^{o}-E_{\gamma}^{o}}+\frac{1}{E_{b}^{o}-E_{\gamma}^{o}}\right)
\end{aligned}
$$

State $\gamma$ represents an intermediate class 2 state, and $a$ and $b$ represent the class 1 states in resonance. Considering four distinct modes involved in a generalized 2-2 resonance, the total change in quanta in Equation (96) is four. Given the sums above have no restrictions on modes $k l m$ and $k^{\prime} l ' m$ ', many intermediate states are possible that connect the class 1 states $a$ and $b$.

It is convenient to categorize these states according to the number of resonant modes whose quanta change in each bra-ket of Equation (96). Since each bra-ket is a cubic term, the quanta in all four modes involved in the resonance cannot change in one bra-ket. Thus the possibly nonzero terms have the corresponding force constants $\phi_{k l m} \phi_{n r r}$ or $\phi_{k l r} \phi_{m n r}$, where 
$k l m n$ are the modes in resonance and $r$ is an arbitrary mode which may or may not equal $k, l$, $m$, or $n$.

In the first term type $\phi_{k l m} \phi_{n r r}$, since the quanta in three of the modes can change by one for each mode in one bra-ket, and the quanta in the fourth mode can change by one in the other bra-ket. The matrix elements for such terms are given in the supplemental material. These matrix elements cancel each other out, leading to a zero contribution from the second order cubic correction. The details of this cancellation are shown in the supplemental material.

In the second term type $\phi_{k l r} \phi_{m n r}$, the quanta of two modes change by one quantum in each bra-ket, a nonzero contribution is obtained. Since there are $\left(\begin{array}{l}4 \\ 2\end{array}\right)=\frac{4 !}{2 ! 2 !}=6$ unique ways for pairs of quanta to change klrlmnr $\quad m n r l k l r \quad k m r l l n r \quad$ lnrlkmr $\quad k n r l l m r \quad l m r l k n r$ where the first two letters represent the modes whose quanta change in the first bra-ket. Additionally, for each arrangement, there are two possible intermediate states $\gamma$ between $a$ and $b$, one of which involves an increase in some mode $r$ by one quantum and one that involves a decrease in mode $r$ by one quantum. The matrix elements are given in the supplemental material for both paths of all six types.

Collecting the matrix elements with the same force constants, the cubic contribution to $\widetilde{H}^{\prime \prime}$ can be formulated and simplified. This process is presented in the supplemental material. The result considering only one intermediate mode $r$ is 


$$
\begin{aligned}
& \frac{1}{4}\left[\left(n_{k}+1\right)\left(n_{l}+1\right)\left(n_{m}+1\right)\left(n_{n}+1\right)\right]^{1 / 2} \cdot\left\{-h c \frac{1}{4} \phi_{k l r} \phi_{m n r} \cdot\left[\frac{1}{-\omega_{k}-\omega_{l}+\omega_{r}}+\frac{1}{-\omega_{m}-\omega_{n}+\omega_{r}}\right.\right. \\
& \left.+\frac{1}{\omega_{m}+\omega_{n}+\omega_{r}}+\frac{1}{\omega_{k}+\omega_{l}+\omega_{r}}\right] \\
& -h c \frac{1}{4} \phi_{k m r} \phi_{l n r} \cdot\left[\frac{1}{-\omega_{k}+\omega_{m}+\omega_{r}}+\frac{1}{\omega_{l}-\omega_{n}+\omega_{r}}+\frac{1}{-\omega_{l}+\omega_{n}+\omega_{r}}+\frac{1}{\omega_{k}-\omega_{m}+\omega_{r}}\right] \\
& \left.-h c \frac{1}{4} \phi_{k n r} \phi_{l m r} \cdot\left[\frac{1}{-\omega_{k}+\omega_{n}+\omega_{r}}+\frac{1}{\omega_{l}-\omega_{m}+\omega_{r}}+\frac{1}{-\omega_{l}+\omega_{m}+\omega_{r}}+\frac{1}{\omega_{k}-\omega_{n}+\omega_{r}}\right]\right\}
\end{aligned}
$$

To complete the expression, there must be a sum over all possible intermediate modes $r$ to ensure all modes are accounted for

$$
\begin{aligned}
& \frac{1}{2} \sum_{\gamma}\left\langle a\left|h c \sum_{k l m} \frac{1}{3 !} \phi_{k l m} q_{k} q_{l} q_{m}\right| \gamma\right\rangle\left\langle\gamma\left|h c \sum_{k^{\prime} l^{\prime} m^{3}} \frac{1}{3 !} \phi_{k^{\prime} l^{\prime} m^{\prime}} q_{k^{\prime}} q_{l^{\prime}} q_{m^{\prime}}\right| b\right\rangle\left(\frac{1}{E_{a}^{o}-E_{\gamma}^{o}}+\frac{1}{E_{b}^{o}-E_{\gamma}^{o}}\right) \\
& =\frac{1}{4}\left[\left(n_{k}+1\right)\left(n_{l}+1\right)\left(n_{m}+1\right)\left(n_{n}+1\right)\right]^{1 / 2} \cdot\left\{-h c \frac{1}{4} \sum_{r} \phi_{k l r} \phi_{m n r} \cdot\left[\frac{1}{-\omega_{k}-\omega_{l}+\omega_{r}}+\frac{1}{-\omega_{m}-\omega_{n}+\omega_{r}}\right.\right. \\
& \left.+\frac{1}{\omega_{m}+\omega_{n}+\omega_{r}}+\frac{1}{\omega_{k}+\omega_{l}+\omega_{r}}\right] \\
& -h c \frac{1}{4} \sum_{r} \phi_{k m r} \phi_{l n r} \cdot\left[\frac{1}{\omega_{k}-\omega_{m}+\omega_{r}}+\frac{1}{\omega_{l}-\omega_{n}+\omega_{r}}+\frac{1}{-\omega_{k}+\omega_{m}+\omega_{r}}+\frac{1}{-\omega_{l}+\omega_{n}+\omega_{r}}\right] \\
& \left.-h c \frac{1}{4} \sum_{r} \phi_{k n r} \phi_{l m r} \cdot\left[\frac{1}{\omega_{k}-\omega_{n}+\omega_{r}}+\frac{1}{\omega_{l}-\omega_{m}+\omega_{r}}+\frac{1}{-\omega_{k}+\omega_{n}+\omega_{r}}+\frac{1}{-\omega_{l}+\omega_{m}+\omega_{r}}\right]\right\}
\end{aligned}
$$

Note that the same results are obtained whether or not $r$ equals $k, l, m$, or $n$. The resonance denominators of Equation (97) will be of the same form, and the factor of one quarter will remain constant for each sum. However, the origins of the one quarter are different when $r$ equals $k, l, m$, or $n$. For all $r$, one-half arises from the general form of the correction term, shown in Equation (97). When $r$ is a distinct mode, the other half arises from the matrix element. When $r$ is not a distinct mode, the other half originates in the permutation factor associated with the force constants. For further details regarding these matrix elements, see the supplemental material. 
Combining the quartic, vibrational angular momentum, and cubic contributions, Equations (94), (95), and (97), respectively, the final expression for the off-diagonal matrix element for the general 2-2 resonance becomes

$$
\left\langle\ldots n_{k}+1, n_{l}+1, n_{m}, n_{n} \ldots\left|\tilde{H}^{\prime \prime}\right| . . n_{k}, n_{l}, n_{m}+1, n_{n}+1 \ldots\right\rangle=K_{k l ; m n} \frac{1}{4}\left[\left(n_{k}+1\right)\left(n_{l}+1\right)\left(n_{m}+1\right)\left(n_{n}+1\right)\right]^{1 / 2}
$$

where

$$
\begin{aligned}
& K_{k l ; m n}=h c \phi_{k l m n} \\
& +2 \sum_{\alpha} B_{\alpha}\left[\frac{\xi_{k l}^{\alpha} \xi_{m n}^{\alpha}\left(\omega_{k}-\omega_{l}\right)\left(\omega_{m}-\omega_{n}\right)-\xi_{k m}^{\alpha} \xi_{l n}^{\alpha}\left(\omega_{k}+\omega_{m}\right)\left(\omega_{l}+\omega_{n}\right)-\xi_{k n}^{\alpha} \xi_{l m}^{\alpha}\left(\omega_{k}+\omega_{n}\right)\left(\omega_{l}+\omega_{m}\right)}{\left(\omega_{k} \omega_{l} \omega_{m} \omega_{n}\right)^{1 / 2}}\right] \\
& -h c \frac{1}{4} \sum_{r} \phi_{k l r} \phi_{m n r} \cdot\left[\frac{1}{\omega_{k}+\omega_{l}+\omega_{r}}+\frac{1}{\omega_{m}+\omega_{n}+\omega_{r}}+\frac{1}{-\omega_{k}-\omega_{l}+\omega_{r}}+\frac{1}{-\omega_{m}-\omega_{n}+\omega_{r}}\right] \\
& -h c \frac{1}{4} \sum_{r} \phi_{k m r} \phi_{l n r} \cdot\left[\frac{1}{\omega_{k}-\omega_{m}+\omega_{r}}+\frac{1}{\omega_{l}-\omega_{n}+\omega_{r}}+\frac{1}{-\omega_{k}+\omega_{m}+\omega_{r}}+\frac{1}{-\omega_{l}+\omega_{n}+\omega_{r}}\right] \\
& -h c \frac{1}{4} \sum_{r} \phi_{k n r} \phi_{l m r} \cdot\left[\frac{1}{\omega_{k}-\omega_{n}+\omega_{r}}+\frac{1}{\omega_{l}-\omega_{m}+\omega_{r}}+\frac{1}{-\omega_{k}+\omega_{n}+\omega_{r}}+\frac{1}{-\omega_{l}+\omega_{m}+\omega_{r}}\right]
\end{aligned}
$$

Equation (99) is equivalent to Hänninen and Halonen's Equation (15) [5], Matthew et al.'s Equation (8) [6], and Law's Equation (1.49) [28] but corrects typographical errors in Lehmann's Equation (12) [3] and Martin and Taylor's Equation (11) [4].

The other 2-2 resonances involve constants $K_{k k ; l}$ and $K_{k l ; m m}$

$$
\left\langle\ldots n_{k}+2, n_{l} \ldots\left|\widetilde{H}^{\prime \prime}\right| . . . n_{k}, n_{l}+2 \ldots\right\rangle=K_{k k ; l l} \frac{1}{4}\left[\left(n_{k}+1\right)\left(n_{k}+2\right)\left(n_{l}+1\right)\left(n_{l}+2\right)\right]^{1 / 2}
$$

where 


$$
\begin{aligned}
& K_{k k ; l}=h c \frac{\phi_{k k l l}}{4}-\sum_{\alpha} B_{\alpha} \frac{\left(\zeta_{k l}^{\alpha}\right)^{2}\left(\omega_{k}+\omega_{l}\right)^{2}}{\omega_{k} \omega_{l}} \\
& -h c \frac{1}{16} \sum_{r} \phi_{k k r} \phi_{l l r} \cdot\left[\frac{1}{\omega_{k}+\omega_{k}+\omega_{r}}+\frac{1}{-\omega_{k}-\omega_{k}+\omega_{r}}+\frac{1}{\omega_{l}+\omega_{l}+\omega_{r}}+\frac{1}{-\omega_{l}-\omega_{l}+\omega_{r}}\right] \\
& -h c \frac{1}{4} \sum_{r}\left(\phi_{k l r}\right)^{2} \cdot\left[\frac{1}{\omega_{k}-\omega_{l}+\omega_{r}}+\frac{1}{-\omega_{k}+\omega_{l}+\omega_{r}}\right]
\end{aligned}
$$

and

$$
\left\langle\ldots n_{k}+2, n_{l}, n_{m} \ldots\left|\widetilde{H}^{\prime \prime}\right| \ldots n_{k}, n_{l}+1, n_{m}+1 \ldots\right\rangle=K_{k k: l m} \frac{1}{4}\left[\left(n_{k}+1\right)\left(n_{k}+2\right)\left(n_{l}+1\right)\left(n_{m}+1\right)\right]^{1 / 2}
$$

where

$$
\begin{aligned}
& K_{k k ; m}=h c \frac{\phi_{k k l m}}{2}-2 \sum_{\alpha} B_{\alpha} \frac{\zeta_{k l}^{\alpha} \zeta_{k m}^{\alpha}\left(\omega_{k}+\omega_{l}\right)\left(\omega_{k}+\omega_{m}\right)}{\omega_{k} \sqrt{\omega_{l} \omega_{m}}} \\
& -h c \frac{1}{4} \sum_{r} \phi_{k l r} \phi_{k m r} \cdot\left[\frac{1}{\omega_{k}-\omega_{l}+\omega_{r}}+\frac{1}{-\omega_{k}+\omega_{l}+\omega_{r}}+\frac{1}{\omega_{k}-\omega_{m}+\omega_{r}}+\frac{1}{-\omega_{k}+\omega_{m}+\omega_{r}}\right] \\
& -h c \frac{1}{8} \sum_{r} \phi_{k k r} \phi_{l m r} \cdot\left[\frac{1}{\omega_{k}+\omega_{k}+\omega_{r}}+\frac{1}{-\omega_{k}-\omega_{k}+\omega_{r}}+\frac{1}{\omega_{l}+\omega_{m}+\omega_{r}}+\frac{1}{-\omega_{l}-\omega_{m}+\omega_{r}}\right]
\end{aligned}
$$

These are specific cases of $K_{k l ; m n}$ and can be derived from first principles or from $K_{k l ; m n}$. The derivation for $K_{k k ; l l}$ will be shown below as an example.

b. Derivation of specific case $K_{k k ; l}$ from $K_{k l ; m n}$. It will be shown that Equation (101) follows from Equation (99) when $l=k$ and $n=m$, giving only two distinct modes involved in the resonance.

The general matrix element for this 2-2 resonance is

$$
\left\langle\ldots n_{k}+2, n_{l} \ldots\left|\tilde{H}^{\prime \prime}\right| \ldots n_{k}, n_{l}+2 \ldots\right\rangle=K_{k k ; l l} \frac{1}{4}\left[\left(n_{k}+2\right)\left(n_{k}+1\right)\left(n_{l}+2\right)\left(n_{l}+1\right)\right]^{1 / 2}
$$


Though the matrix element is different in form, it can be shown to be a special case of the generalized 2-2 resonance matrix element.

As with $K_{k l ; m n}$, the quartic contribution is the simplest to evaluate. Given the quartic term in $K_{k l ; m n}$ is $\phi_{k l m n}$, and accounting for the reciprocal factorial and permutation factor, the contribution becomes

$$
\frac{4 !}{2 ! 2 !} \cdot \frac{1}{4 !} \phi_{k k l l}=\frac{1}{4} \phi_{k k l l}
$$

To derive the vibrational angular momentum term from $K_{k l ; m n}$, let $l=k$ and $m=n=l$ :

$$
\begin{aligned}
& 2 \sum_{\alpha} B_{\alpha}\left[\frac{\zeta_{k k}^{\alpha} \zeta_{l l}^{\alpha}\left(\omega_{k}-\omega_{k}\right)\left(\omega_{l}-\omega_{l}\right)-\zeta_{k l}^{\alpha} \zeta_{k l}^{\alpha}\left(\omega_{k}+\omega_{l}\right)\left(\omega_{k}+\omega_{l}\right)-\zeta_{k l}^{\alpha} \zeta_{k l}^{\alpha}\left(\omega_{k}+\omega_{l}\right)\left(\omega_{k}+\omega_{l}\right)}{\left(\omega_{k} \omega_{k} \omega_{l} \omega_{l}\right)^{1 / 2}}\right] \\
& =2 \sum_{\alpha} B_{\alpha}\left[\frac{0-\left(\zeta_{k l}^{\alpha}\right)^{2}\left(\omega_{k}+\omega_{l}\right)^{2}-\left(\zeta_{k l}^{\alpha}\right)^{2}\left(\omega_{k}+\omega_{l}\right)^{2}}{\omega_{k} \omega_{l}}\right]
\end{aligned}
$$

Note that there are $4 !=24$ possible arrangements of the indices $k l m n$ but only

$\frac{4 !}{2 ! 2 !}=6$ arrangements of $k k l l$, where the arrangements $k k l l$ and $l l k k$ give zero-valued matrix elements since $\zeta_{i i}^{\alpha}=0$. Thus to account for the proper number of arrangements, the vibrational angular momentum term is divided by four to obtain

$$
\frac{1}{4}\left\{2 \sum_{\alpha} B_{\alpha}\left[\frac{-2\left(\zeta_{k l}^{\alpha}\right)^{2}\left(\omega_{k}+\omega_{l}\right)^{2}}{\omega_{k} \omega_{l}}\right]\right\}=-\sum_{\alpha} B_{\alpha}\left[\frac{\left(\zeta_{k l}^{\alpha}\right)^{2}\left(\omega_{k}+\omega_{l}\right)^{2}}{\omega_{k} \omega_{l}}\right]
$$

The second order cubic correction follows in a similar manner: 


$$
\begin{aligned}
& -h c \frac{1}{4} \sum_{r} \frac{3 !}{2 ! ! !} \cdot \frac{1}{3 !} \phi_{k k r} \frac{3 !}{2 ! ! !} \cdot \frac{1}{3 !} \phi_{l l r} \cdot\left[\frac{1}{\omega_{k}+\omega_{k}+\omega_{r}}+\frac{1}{\omega_{l}+\omega_{l}+\omega_{r}}+\frac{1}{-\omega_{k}-\omega_{k}+\omega_{r}}+\frac{1}{-\omega_{l}-\omega_{l}+\omega_{r}}\right] \\
& -h c \frac{1}{4} \sum_{r} \frac{3 !}{1 ! 1 ! !} \cdot \frac{1}{3 !} \phi_{k l r} \frac{3 !}{1 ! 1 ! !} \cdot \frac{1}{3 !} \phi_{k l r} \cdot\left[\frac{1}{\omega_{k}-\omega_{l}+\omega_{r}}+\frac{1}{\omega_{k}-\omega_{l}+\omega_{r}}+\frac{1}{-\omega_{k}+\omega_{l}+\omega_{r}}+\frac{1}{-\omega_{k}+\omega_{l}+\omega_{r}}\right] \\
& -h c \frac{1}{4} \sum_{r} \frac{3 !}{1 ! 1 ! !} \cdot \frac{1}{3 !} \phi_{k l r} \frac{3 !}{1 ! 1 ! !} \cdot \frac{1}{3 !} \phi_{k l r} \cdot\left[\frac{1}{\omega_{k}-\omega_{l}+\omega_{r}}+\frac{1}{\omega_{k}-\omega_{l}+\omega_{r}}+\frac{1}{-\omega_{k}+\omega_{l}+\omega_{r}}+\frac{1}{-\omega_{k}+\omega_{l}+\omega_{r}}\right] \\
& =-h c \frac{1}{16} \sum_{r} \phi_{k k r} \phi_{l l r} \cdot\left[\frac{1}{\omega_{k}+\omega_{k}+\omega_{r}}+\frac{1}{\omega_{l}+\omega_{l}+\omega_{r}}+\frac{1}{-\omega_{k}-\omega_{k}+\omega_{r}}+\frac{1}{-\omega_{l}-\omega_{l}+\omega_{r}}\right] \\
& -h c \frac{2}{4} \sum_{r} \phi_{k l r} \phi_{k l r} \cdot\left[\frac{2}{\omega_{k}-\omega_{l}+\omega_{r}}+\frac{2}{-\omega_{k}+\omega_{l}+\omega_{r}}\right]
\end{aligned}
$$

Note that for the $\phi_{k l r} \phi_{k l r}$ terms in $K_{k k ; l}$, there are two contributing matrix elements, but for the $\phi_{k m r} \phi_{l n r}$ and $\phi_{k n r} \phi_{l m r}$ terms in $K_{k l ; m n}$, there are eight total contributing matrix elements, four for each pair of force constants. Then to account for the difference in number of connecting intermediate states, the last term above is divided by four to give the total second order cubic result

$$
\begin{aligned}
& -h c \frac{1}{16} \sum_{r} \phi_{k k r} \phi_{l l r} \cdot\left[\frac{1}{\omega_{k}+\omega_{k}+\omega_{r}}+\frac{1}{\omega_{l}+\omega_{l}+\omega_{r}}+\frac{1}{-\omega_{k}-\omega_{k}+\omega_{r}}+\frac{1}{-\omega_{l}-\omega_{l}+\omega_{r}}\right] \\
& -h c \frac{1}{4} \sum_{r} \phi_{k l r} \phi_{k l r} \cdot\left[\frac{1}{\omega_{k}-\omega_{l}+\omega_{r}}+\frac{1}{-\omega_{k}+\omega_{l}+\omega_{r}}\right]
\end{aligned}
$$

Combining the three terms, the resonance constant $K_{k k ; l l}$ for the 2-2 resonance becomes Equation (101).

An alternative means to derive $K_{k k ; l}$ is from $K_{k l ; m n}$ by simply considering the different permutation factor associated with the quartic force constant and scale the rest of the resonance constant appropriately. In this case, the permutation factor for $\phi_{k k l l}$ is $\frac{1}{4}$, and thus all terms can be effectively divided by four after making the appropriate substitutions and 
algebraic manipulations in each term. This gives the same result, though by a less rigorous procedure.

\section{1-3 resonances}

The most general 1-3 resonance involves the annihilation of one quantum in one mode and the creation of one quantum in three distinct modes. This said, using Table 1, the most general matrix element of a 1-3 resonance

$$
\left\langle n_{k}+1, n_{l}, n_{m}, n_{n}|\tilde{H}| n_{k}, n_{l}+1, n_{m}+1, n_{n}+1\right\rangle
$$

will be derived, where $k \neq l \neq m \neq n$. The derivation for the 1-3 off-diagonal matrix element will be completed using the process in IV.B.3.

The quartic potential term again is the easiest to evaluate

$$
\begin{aligned}
& \left\langle n_{k}+1, n_{l}, n_{m}, n_{n}\left|h c \sum_{k l m n} \frac{1}{4 !} \phi_{k l m n} q_{k} q_{l} q_{m} q_{n}\right| n_{k}, n_{l}+1, n_{m}+1, n_{n}+1\right\rangle \\
& =h c \frac{4 !}{1 ! 1 ! 1 ! !} \cdot \frac{1}{4 !} \phi_{k l m n} \frac{1}{4}\left[\left(n_{k}+1\right)\left(n_{l}+1\right)\left(n_{m}+1\right)\left(n_{n}+1\right)\right]^{1 / 2} \\
& =h c \phi_{k l m n} \frac{1}{4}\left[\left(n_{k}+1\right)\left(n_{l}+1\right)\left(n_{m}+1\right)\left(n_{n}+1\right)\right]^{1 / 2}
\end{aligned}
$$

The vibrational angular momentum contribution will be derived in a process similar to the one performed for the 2-2 resonance. As described in IV.B.3.a, there are twenty-four arrangements of four distinct letters. If the letters represent the number of quanta in modes $k l m n$, then each arrangement can represent a pair of Coriolis coupling pairs. As explained previously, there are twelve unique arrangements of the four distinct modes. These arrangements' matrix elements and Coriolis constants for these arrangements are presented in the supplemental material.

Combining terms with like Coriolis coupling constants, the vibrational angular momentum matrix element becomes

$$
\left\langle n_{k}+1, n_{l}, n_{m}, n_{n}\left|\sum_{\alpha} B_{\alpha}\left(\sum_{k l} \zeta_{k l}^{\alpha}\left(\frac{\omega_{l}}{\omega_{k}}\right)^{1 / 2} q_{k} p_{l}\right)\left(\sum_{k^{\prime} l^{\prime}} \zeta_{k^{\prime} l^{\prime}}^{\alpha}\left(\frac{\omega_{l^{\prime}}}{\omega_{k^{\prime}}}\right)^{1 / 2} q_{k^{\prime}} p_{l^{\prime}}\right)\right| n_{k}, n_{l}+1, n_{m}+1, n_{n}+1\right\rangle
$$




$$
\begin{aligned}
& =\frac{1}{4}\left[\left(n_{k}+1\right)\left(n_{l}+1\right)\left(n_{m}+1\right)\left(n_{n}+1\right)\right]^{1 / 2} \\
& \cdot 2 \sum_{\alpha} B_{\alpha}\left\{\zeta_{k l}^{\alpha} \zeta_{m n}^{\alpha}\left[-\left(\frac{\omega_{l} \omega_{n}}{\omega_{k} \omega_{m}}\right)^{1 / 2}+\left(\frac{\omega_{k} \omega_{m}}{\omega_{l} \omega_{n}}\right)^{1 / 2}+\left(\frac{\omega_{l} \omega_{m}}{\omega_{k} \omega_{n}}\right)^{1 / 2}-\left(\frac{\omega_{k} \omega_{n}}{\omega_{l} \omega_{m}}\right)^{1 / 2}\right]\right. \\
& +\zeta_{k m}^{\alpha} \zeta_{l n}^{\alpha}\left[-\left(\frac{\omega_{m} \omega_{n}}{\omega_{k} \omega_{l}}\right)^{1 / 2}+\left(\frac{\omega_{k} \omega_{l}}{\omega_{m} \omega_{n}}\right)^{1 / 2}+\left(\frac{\omega_{l} \omega_{m}}{\omega_{k} \omega_{n}}\right)^{1 / 2}-\left(\frac{\omega_{k} \omega_{n}}{\omega_{l} \omega_{m}}\right)^{1 / 2}\right] \\
& \left.+\zeta_{k n}^{\alpha} \zeta_{l m}^{\alpha}\left[\left(\frac{\omega_{l} \omega_{n}}{\omega_{k} \omega_{m}}\right)^{1 / 2}-\left(\frac{\omega_{k} \omega_{m}}{\omega_{l} \omega_{n}}\right)^{1 / 2}-\left(\frac{\omega_{m} \omega_{n}}{\omega_{k} \omega_{l}}\right)^{1 / 2}+\left(\frac{\omega_{k} \omega_{l}}{\omega_{m} \omega_{n}}\right)^{1 / 2}\right]\right\} \\
& =\frac{1}{4}\left[\left(n_{k}+1\right)\left(n_{l}+1\right)\left(n_{m}+1\right)\left(n_{n}+1\right)\right]^{1 / 2} \cdot 2 \sum_{\alpha} B_{\alpha}\left\{\zeta_{k l}^{\alpha} \zeta_{m n}^{\alpha}\left[\frac{-\omega_{l} \omega_{n}+\omega_{k} \omega_{m}+\omega_{l} \omega_{m}-\omega_{k} \omega_{n}}{\left(\omega_{k} \omega_{l} \omega_{m} \omega_{n}\right)^{1 / 2}}\right]\right. \\
& +\zeta_{k m}^{\alpha} \zeta_{l n}^{\alpha}\left[\frac{-\omega_{m} \omega_{n}+\omega_{k} \omega_{l}+\omega_{l} \omega_{m}-\omega_{k} \omega_{n}}{\left(\omega_{k} \omega_{l} \omega_{m} \omega_{n}\right)^{1 / 2}}+\zeta_{k n}^{\alpha} \zeta_{l m}^{\alpha}\left[\frac{\omega_{l} \omega_{n}-\omega_{k} \omega_{m}-\omega_{m} \omega_{n}+\omega_{k} \omega_{l}}{\left(\omega_{k} \omega_{l} \omega_{m} \omega_{n}\right)^{1 / 2}}\right]\right\} \\
& =\frac{1}{4}\left[\left(n_{k}+1\right)\left(n_{l}+1\right)\left(n_{m}+1\right)\left(n_{n}+1\right)\right]^{1 / 2} . \\
& 2 \sum_{\alpha} B_{\alpha}\left[\frac{\zeta_{k l}^{\alpha} \zeta_{m n}^{\alpha}\left(\omega_{k}+\omega_{l}\right)\left(\omega_{m}-\omega_{n}\right)+\zeta_{k m}^{\alpha} \zeta_{l n}^{\alpha}\left(\omega_{k}+\omega_{m}\right)\left(\omega_{l}-\omega_{n}\right)+\zeta_{k n}^{\alpha} \zeta_{m l}^{\alpha}\left(\omega_{k}+\omega_{n}\right)\left(\omega_{l}-\omega_{m}\right)}{\left(\omega_{k} \omega_{l} \omega_{m} \omega_{n}\right)^{1 / 2}}\right]
\end{aligned}
$$

Lastly, the cubic potential contribution will be derived. As with the general 2-2

resonance, nonzero contributions arise when the quanta of two of the modes involved in the resonance change in each bra-ket. In the first bra-ket of Equation (96), for the general 1-3 resonance, the quanta in two modes in state $a$ will change by one quantum for each mode, and the quanta in some mode $r$ will change by one quantum. In the second bra-ket of Equation (96), the quanta in the other two modes will change by one quantum for each mode, and the quanta in mode $r$ will return to the original quanta. As in IV.B.3.a, there are only six unique ways for pairs of quanta to change

$$
\text { kllmn } \mathrm{mn} / \mathrm{kl} \quad \mathrm{km} / \mathrm{ln} \ln / \mathrm{km} \quad \mathrm{kn} / \mathrm{lm} \mathrm{lm} / \mathrm{kn}
$$

where the first two letters represent the modes whose quanta change in the first bra-ket. 
As seen previously, for each of the three sets of two arrangements, there are two possible interactions between states $a$ and $b$, one increasing quanta in mode $r$ by one quantum and one decreasing quanta in mode $r$ by one quantum. To more fully illustrate this, the matrix elements and constants are given in the supplemental material for both paths of all six types.

The result formulated considering only one intermediate mode $r$ is

$$
\begin{aligned}
& \frac{1}{4}\left[\left(n_{k}+1\right)\left(n_{l}+1\right)\left(n_{m}+1\right)\left(n_{n}+1\right)\right]^{1 / 2}\left\{-h c \frac{1}{4} \phi_{k l r} \phi_{m n r} \cdot\left[\frac{1}{-\omega_{k}+\omega_{l}+\omega_{r}}+\frac{1}{-\omega_{m}-\omega_{n}+\omega_{r}}\right.\right. \\
& \left.+\frac{1}{\omega_{m}+\omega_{n}+\omega_{r}}+\frac{1}{\omega_{k}-\omega_{l}+\omega_{r}}\right] \\
& -h c \frac{1}{4} \phi_{k m r} \phi_{l n r} \cdot\left[\frac{1}{-\omega_{k}+\omega_{m}+\omega_{r}}+\frac{1}{-\omega_{l}-\omega_{n}+\omega_{r}}+\frac{1}{\omega_{l}+\omega_{n}+\omega_{r}}+\frac{1}{\omega_{k}-\omega_{m}+\omega_{r}}\right] \\
& \left.-h c \frac{1}{4} \phi_{k n r} \phi_{l m r} \cdot\left[\frac{1}{-\omega_{k}+\omega_{n}+\omega_{r}}+\frac{1}{-\omega_{l}-\omega_{m}+\omega_{r}}+\frac{1}{\omega_{l}+\omega_{m}+\omega_{r}}+\frac{1}{\omega_{k}-\omega_{n}+\omega_{r}}\right]\right\}
\end{aligned}
$$

To complete the expression, there must be a sum over all possible intermediate modes $r$ to ensure all modes are accounted for

$$
\begin{aligned}
& \frac{1}{2} \sum_{\gamma}\left\langle a\left|h c \sum_{k l m} \frac{1}{3 !} \phi_{k l m} q_{k} q_{l} q_{m}\right| \gamma\right\rangle\left\langle\gamma\left|h c \sum_{k^{\prime} l^{\prime} m^{\prime}} \frac{1}{3 !} \phi_{k^{\prime} l^{\prime} m^{\prime}} q_{k^{\prime}} q_{l^{\prime}} q_{m^{\prime}}\right| b\right\rangle\left(\frac{1}{E_{a}^{o}-E_{\gamma}^{o}}+\frac{1}{E_{b}^{o}-E_{\gamma}^{o}}\right)= \\
& \frac{1}{4}\left[\left(n_{k}+1\right)\left(n_{l}+1\right)\left(n_{m}+1\right)\left(n_{n}+1\right)\right]^{1 / 2}\left\{-h c \frac{1}{4} \sum_{r} \phi_{k l r} \phi_{m n r} \cdot\left[\frac{1}{-\omega_{k}+\omega_{l}+\omega_{r}}+\frac{1}{-\omega_{m}-\omega_{n}+\omega_{r}}\right.\right. \\
& \left.+\frac{1}{\omega_{m}+\omega_{n}+\omega_{r}}+\frac{1}{\omega_{k}-\omega_{l}+\omega_{r}}\right] \\
& -h c \frac{1}{4} \sum_{r} \phi_{k m r} \phi_{l n r} \cdot\left[\frac{1}{\omega_{k}-\omega_{m}+\omega_{r}}+\frac{1}{\omega_{l}+\omega_{n}+\omega_{r}}+\frac{1}{-\omega_{k}+\omega_{m}+\omega_{r}}+\frac{1}{-\omega_{l}-\omega_{n}+\omega_{r}}\right] \\
& \left.-h c \frac{1}{4} \sum_{r} \phi_{k n r} \phi_{l m r} \cdot\left[\frac{1}{\omega_{k}-\omega_{n}+\omega_{r}}+\frac{1}{\omega_{l}+\omega_{m}+\omega_{r}}+\frac{1}{-\omega_{k}+\omega_{n}+\omega_{r}}+\frac{1}{-\omega_{l}-\omega_{m}+\omega_{r}}\right]\right\}
\end{aligned}
$$

Note that for the reasons given in the general 2-2 derivation, the weighting of each term is the same when $r=k, l, m$, or $n$. 
Combining the quartic, vibrational angular momentum, and cubic contributions, given in Equations (104), (105), and (106), respectively, the final expression for the off-diagonal matrix element for the general 1-3 resonance becomes

$$
\left\langle. . n_{k}+1, n_{l}, n_{m}, n_{n} \ldots\left|\widetilde{H}^{\prime \prime}\right| . . n_{k}, n_{l}+1, n_{m}+1, n_{n}+1 \ldots\right\rangle=K_{k ; \operatorname{lnn} n} \frac{1}{4}\left[\left(n_{k}+1\right)\left(n_{l}+1\right)\left(n_{m}+1\right)\left(n_{n}+1\right)\right]^{1 / 2}
$$

where

$$
\begin{aligned}
& K_{k: l m n}=h c \phi_{k l m n}+2 \sum_{\alpha} B_{\alpha}\left[\frac{\zeta_{k l}^{\alpha} \zeta_{m n}^{\alpha}\left(\omega_{k}+\omega_{l}\right)\left(\omega_{m}-\omega_{n}\right)+\zeta_{k m}^{\alpha} \zeta_{l n}^{\alpha}\left(\omega_{k}+\omega_{m}\right)\left(\omega_{l}-\omega_{n}\right)+\zeta_{k n}^{\alpha} \xi_{m l}^{\alpha}\left(\omega_{k}+\omega_{n}\right)\left(\omega_{l}-\omega_{m}\right)}{\left(\omega_{k} \omega_{l} \omega_{m} \omega_{n}\right)^{1 / 2}}\right] \\
& -h c \frac{1}{4} \sum_{r} \phi_{k l r} \phi_{m n r} \cdot\left[\frac{1}{-\omega_{k}+\omega_{l}+\omega_{r}}+\frac{1}{-\omega_{m}-\omega_{n}+\omega_{r}}+\frac{1}{\omega_{k}-\omega_{l}+\omega_{r}}+\frac{1}{\omega_{m}+\omega_{n}+\omega_{r}}\right] \\
& -h c \frac{1}{4} \sum_{r} \phi_{k m r} \phi_{l n r} \cdot\left[\frac{1}{\omega_{k}-\omega_{m}+\omega_{r}}+\frac{1}{\omega_{l}+\omega_{n}+\omega_{r}}+\frac{1}{-\omega_{k}+\omega_{m}+\omega_{r}}+\frac{1}{-\omega_{l}-\omega_{n}+\omega_{r}}\right] \\
& -h c \frac{1}{4} \sum_{r} \phi_{k n r} \phi_{l m r} \cdot\left[\frac{1}{\omega_{k}-\omega_{n}+\omega_{r}}+\frac{1}{\omega_{l}+\omega_{m}+\omega_{r}}+\frac{1}{-\omega_{k}+\omega_{n}+\omega_{r}}+\frac{1}{-\omega_{l}-\omega_{m}+\omega_{r}}\right]
\end{aligned}
$$

Equation (108) corrects sign errors in Martin and Taylor's Equation (20) [4].

The other 1-3 resonance constants, $K_{k ; l l l}$ and $K_{k ; l m m}$, are specific cases of $K_{k ; l m n}$ and can be derived directly from $K_{k ; m n}$ using a process similar to the one followed in IV.B.3.b.

Consequently, their derivations will not be presented here. They are

$$
\left\langle\ldots n_{k}+1, n_{l}, n_{m} \ldots\left|\tilde{H}^{\prime \prime}\right| . . n_{k}, n_{l}+2, n_{m}+1 \ldots\right\rangle=K_{k ; l l m} \frac{1}{4}\left[\left(n_{k}+1\right)\left(n_{l}+1\right)\left(n_{l}+2\right)\left(n_{m}+1\right)\right]^{1 / 2}
$$

where 


$$
\begin{aligned}
& K_{k: l l m}=h c \frac{\phi_{k l l m}}{2}+2 \sum_{\alpha} B_{\alpha}\left[\frac{\xi_{k l}^{\alpha} \zeta_{l m}^{\alpha}\left(\omega_{k}+\omega_{l}\right)\left(\omega_{l}-\omega_{m}\right)}{\omega_{l}\left(\omega_{k} \omega_{m}\right)^{1 / 2}}\right] \\
& -h c \frac{1}{4} \sum_{r} \phi_{k l r} \phi_{l m r} \cdot\left[\frac{1}{-\omega_{k}+\omega_{l}+\omega_{r}}+\frac{1}{\omega_{k}-\omega_{l}+\omega_{r}}+\frac{1}{\omega_{l}+\omega_{m}+\omega_{r}}+\frac{1}{-\omega_{l}-\omega_{m}+\omega_{r}}\right] \\
& -h c \frac{1}{8} \sum_{r} \phi_{k m r} \phi_{l l r} \cdot\left[\frac{1}{\omega_{l}+\omega_{l}+\omega_{r}}+\frac{1}{-\omega_{l}-\omega_{l}+\omega_{r}}+\frac{1}{\omega_{k}-\omega_{m}+\omega_{r}}+\frac{1}{-\omega_{k}+\omega_{m}+\omega_{r}}\right]
\end{aligned}
$$

and

$$
\left\langle\ldots n_{k}+1, n_{l} \ldots\left|\widetilde{H}^{\prime \prime}\right| \ldots n_{k}, n_{l}+3 \ldots\right\rangle=K_{k ; l l l} \frac{1}{4}\left[\left(n_{k}+1\right)\left(n_{l}+1\right)\left(n_{l}+2\right)\left(n_{l}+3\right)\right]^{1 / 2}
$$

where

$$
\begin{aligned}
& K_{k ; l l l}=h c \frac{\phi_{k l l l}}{6} \\
& -h c \frac{1}{8} \sum_{r} \phi_{k l r} \phi_{l l r} \cdot\left[\frac{1}{\omega_{k}-\omega_{m}+\omega_{r}}+\frac{1}{-\omega_{k}+\omega_{m}+\omega_{r}}+\frac{1}{\omega_{l}+\omega_{l}+\omega_{r}}+\frac{1}{-\omega_{l}-\omega_{l}+\omega_{r}}\right]
\end{aligned}
$$

These correspond to Equations (21) and (22), respectively, of Martin and Taylor, correcting for typographical errors [4]. Lehmann, Matthews et al., Hänninen and Halonen, and Law do not consider $1-3$ resonances $[3,5,6,28]$.

\section{1-1 Resonances}

The final type of resonance that can be treated in second order is the 1-1 resonance. The matrix element for a 1-1 resonance is

$$
\left\langle n_{k}+1, n_{m}, n_{l}|\tilde{H}| n_{k}, n_{m}+1, n_{l}\right\rangle
$$

Despite a change of only two quantum numbers, there is no harmonic contribution because harmonic force constants are diagonal for normal mode vibrations, i.e., $\phi_{k m}=0$ for $k$ $\neq m$. Since the overall number of quanta in mode $l$ does not change, there are many possible 
intermediate states that connect class 1 states $a$ and $b$. A convenient means to categorize these states can begin with considering possible cases for mode $l$ : first, $l \neq k$ or $m$; second, $l=k$; third, $l=m$.

There are two types of resonance constants, $K_{k m ; m m}$ and $K_{k l ; m l}$, that arise from this matrix element, and one type could be considered a "special case" of the other. However, since the two occur simultaneously in the full treatment of a 1-1 resonance, the derivations of both resonance constants need to be presented.

There is no first order cubic correction. Since only two modes are involved in the resonance, not three, the matrix element is zero.

The quartic correction term can be derived in a manner similar to the procedure in IV.B.2 and IV.B.3. In the first case, where $l \neq k$ or $m$, we have

$$
\begin{aligned}
& \sum_{l}\left\langle n_{k}+1, n_{m}, n_{l}\left|h c \sum_{k l m n} \frac{1}{4 !} \phi_{k l m n} q_{k} q_{l} q_{m} q_{n}\right| n_{k}, n_{m}+1, n_{l}\right\rangle \\
& =\sum_{l}\left\langle n_{k}+1, n_{m}, n_{l}\left|h c \sum_{k m l} \frac{4 !}{1 ! 1 ! 2 !} \cdot \frac{1}{4 !} \phi_{k l m l} q_{k} q_{m} q_{l}^{2}\right| n_{k}, n_{m}+1, n_{l}\right\rangle \\
& =\frac{1}{2}\left[\left(n_{k}+1\right)\left(n_{m}+1\right)\right]^{1 / 2} \sum_{l}\left(n_{l}+\frac{1}{2}\right) \cdot \frac{1}{2} h c \phi_{k l m l}
\end{aligned}
$$

Note there is a sum over $l$ since there are $(3 N-6)-2=3 N-8$ possible modes $l$. In the second case, $l=k$; so then we have 


$$
\begin{aligned}
& \left\langle n_{k}+1, n_{m}, n_{k}\left|h c \sum_{k l m n} \frac{1}{4 !} \phi_{k l m n} q_{k} q_{l} q_{m} q_{n}\right| n_{k}, n_{m}+1, n_{k}\right\rangle \\
& =\left\langle n_{k}+1, n_{m}, n_{k}\left|h c \sum_{k l m n, l=k} \frac{4 !}{1 ! 3 !} \cdot \frac{1}{4 !} \phi_{k m k k} q_{k} q_{m} q_{k}^{2}\right| n_{k}, n_{m}+1, n_{k}\right\rangle \\
& =\left\langle n_{k}+1, n_{m}, n_{k}\left|h c \sum_{k l m n, l=k} \frac{4 !}{1 ! 3 !} \cdot \frac{1}{4 !} \phi_{m k k k} q_{k}^{3} q_{m}\right| n_{k}, n_{m}+1, n_{k}\right\rangle \\
& =\frac{1}{3 !} h c \phi_{m k k k}\left\langle n_{k}+1\left|q_{k}^{3}\right| n_{k}\right\rangle\left\langle n_{m}\left|q_{m}\right| n_{m}+1\right\rangle\left\langle n_{k} \mid n_{k}\right\rangle \\
& =\frac{1}{3 !} h c \phi_{m k k k} \frac{3}{2}\left[\frac{\left(n_{k}+1\right)^{3}}{2}\right]^{1 / 2}\left[\frac{\left(n_{m}+1\right)}{2}\right]^{1 / 2}(1) \\
& =h c \frac{1}{6} \phi_{m k k k} 3 \cdot \frac{\left[\left(n_{k}+1\right)\left(n_{m}+1\right)\right]^{1 / 2}}{4}\left(n_{k}+1\right) \\
& =3 \cdot \frac{\left[\left(n_{k}+1\right)\left(n_{m}+1\right)\right]^{1 / 2}}{4}\left(n_{k}+1\right) \cdot h c \frac{1}{6} \phi_{m k k k} \\
& =\frac{\left[\left(n_{k}+1\right)\left(n_{m}+1\right)\right]^{1 / 2}}{4}\left(n_{k}+1\right) \cdot h c \frac{1}{2} \phi_{m k k k}
\end{aligned}
$$

Similarly, in the third case, $l=m$, we have

$$
\begin{aligned}
& \left\langle n_{k}+1, n_{m}, n_{m}\left|h c \sum_{k l m n} \frac{1}{4 !} \phi_{k l m n} q_{k} q_{l} q_{m} q_{n}\right| n_{k}, n_{m}+1, n_{m}\right\rangle \\
& =\left\langle n_{k}+1, n_{m}, n_{m}\left|h c \sum_{k l m n, l=m} \frac{4 !}{13 !} \cdot \frac{1}{4 !} \phi_{k m m m} q_{k} q_{m} q_{m}^{2}\right| n_{k}, n_{m}+1, n_{m}\right\rangle \\
& =\left\langle n_{k}+1, n_{m}, n_{m}\left|h c \sum_{k l m n n, l=m} \frac{4 !}{1 ! 3 !} \cdot \frac{1}{4 !} \phi_{k m m m} q_{k} q_{m}^{3}\right| n_{k}, n_{m}+1, n_{m}\right\rangle \\
& =h c \frac{1}{3 !} \phi_{k m m m}\left\langle n_{m}+1\left|q_{m}^{3}\right| n_{m}\right\rangle\left\langle n_{k}\left|q_{k}\right| n_{k}+1\right\rangle\left\langle n_{m} \mid n_{m}\right\rangle \\
& =h c \frac{1}{3 !} \phi_{k m m m}\left[\frac{\left(n_{k}+1\right)}{2}\right]^{1 / 2} \frac{3}{2}\left[\frac{\left(n_{m}+1\right)^{3}}{2}\right]^{1 / 2}(1) \\
& =h c \frac{1}{6} \phi_{k m m m} 3 \cdot \frac{\left[\left(n_{k}+1\right)\left(n_{m}+1\right)\right]^{1 / 2}}{4}\left(n_{m}+1\right) \\
& =3 \cdot \frac{\left[\left(n_{k}+1\right)\left(n_{m}+1\right)\right]^{1 / 2}}{4}\left(n_{m}+1\right) \cdot h c \frac{1}{6} \phi_{k m m m} \\
& =\frac{\left[\left(n_{k}+1\right)\left(n_{m}+1\right)\right]^{1 / 2}}{4}\left(n_{m}+1\right) \cdot h c \frac{1}{2} \phi_{k m m m}
\end{aligned}
$$


The vibrational angular momentum term arises similarly as well. For the first case, consider the $\frac{4 !}{1 ! 1 ! 2 !}=12$ ways to arrange the indices $k \operatorname{lml}$

$\begin{array}{cc}\text { klml } & \text { mlkl } \\ \text { kllm } & \text { lmkl } \\ l k m l & m l l k \\ l k l m & l m l k \\ \text { kmll } & l l k m \\ m k l l & l l m k\end{array}$

Of the twelve arrangements, only eight will give nonzero results since $\zeta_{l l}^{\alpha}=0$. The matrix elements for the eight nonzero terms are given in the supplemental material. Note that there are four terms that do not have the same matrix element dependence as the quartic contribution in Equation (114). Nevertheless, these terms cancel each other out. The four remaining non-cancelling terms are paired, with only two vibrational angular momentum terms being unique. These remaining terms can be combined as follows to give the vibrational angular momentum contribution

$$
\begin{aligned}
& 2\left\{\frac{1}{2}\left[\left(n_{k}+1\right)\left(n_{m}+1\right)\right]^{1 / 2}\left(n_{l}+\frac{1}{2}\right) \cdot \sum_{\alpha} B_{\alpha}\left[\zeta_{k l}^{\alpha} \zeta_{m l}^{\alpha} \frac{\omega_{l}}{\left(\omega_{k} \omega_{m}\right)^{1 / 2}}\right]\right. \\
& \left.+\frac{1}{2}\left[\left(n_{k}+1\right)\left(n_{m}+1\right)\right]^{1 / 2}\left(n_{l}+\frac{1}{2}\right) \cdot \sum_{\alpha} B_{\alpha}\left[\zeta_{k l}^{\alpha} \zeta_{m l}^{\alpha} \frac{\left(\omega_{k} \omega_{m}\right)^{1 / 2}}{\omega_{l}}\right]\right\} \\
& =\frac{1}{2}\left[\left(n_{k}+1\right)\left(n_{m}+1\right)\right]^{1 / 2}\left(n_{l}+\frac{1}{2}\right) \cdot 2 \sum_{\alpha} B_{\alpha} \zeta_{k l}^{\alpha} \zeta_{m l}^{\alpha}\left[\frac{\omega_{l}}{\left(\omega_{k} \omega_{m}\right)^{1 / 2}}+\frac{\left(\omega_{k} \omega_{m}\right)^{1 / 2}}{\omega_{l}}\right] \\
& =\frac{1}{2}\left[\left(n_{k}+1\right)\left(n_{m}+1\right)\right]^{1 / 2}\left(n_{l}+\frac{1}{2}\right) \cdot 2 \sum_{\alpha} B_{\alpha} \frac{\zeta_{k l}^{\alpha} \zeta_{m l}^{\alpha}\left(\omega_{l}^{2}+\omega_{k} \omega_{m}\right)}{\omega_{l}\left(\omega_{k} \omega_{m}\right)^{1 / 2}}
\end{aligned}
$$

For the second case, consider the $\frac{4 !}{13 !}=4$ ways to arrange the indices $\mathrm{kkkm}$ 


\section{$k k k m \quad m k k k$ \\ $k k m k \quad k m k k$}

None of these cases will lead to nonzero matrix elements since $\xi_{l l}^{\alpha}=0$. Thus the vibrational angular momentum contribution to the 1-1 resonance is zero for the second case. The third case is analogously zero.

Compared to the quartic potential and vibrational angular momentum corrections, the second order cubic correction is much more expansive. Due to the sheer number of possible intermediate states, the process for identifying and evaluating the nonzero matrix elements for the second order cubic correction will be merely outlined, and not all matrix elements will be presented explicitly. The process is essentially the same as for the previous resonances: identification of potentially nonzero contributions, evaluation of matrix elements, combination of like terms according to energy denominators, and factorization of the quantum number dependence.

Let the resonant modes be denoted modes $k$ and $m$. Overall modes $k$ and $m$ change by one quantum each, as prescribed by the resonance. The total degree of $q_{i}$ in modes $k$ and $m$ must be odd since otherwise the quanta could not change by \pm 1 . Then the possible degrees of $q_{i}$ for modes $k$ and $m$ are 1,3, and 5. So the possible combinations of degrees for $q_{k}$ and $q_{m}$ are then 1 and 1, 1 and 3, 3 and 1,3 and 3,1 and 5, and 5 and 1.

In the 1 and 1 case, since the maximum total change of quanta is six in the second order cubic correction, there could be four additional modes whose quanta change. However, since the degree of each $q_{i}$ for these modes would be one, their matrix elements would not be diagonal overall, and so the overall matrix element would be zero. Similarly, if three other modes are considered, one will have degree one, and so the total matrix element would be zero. If there are two other modes considered, only when each mode has $q_{i}$ of degree two (not odd degree) will the matrix element be nonzero. If one other mode is considered, nonzero 
matrix elements could result, since the degree of $q_{i}$ would be four. The force constants associated with these potentially nonzero matrix elements are

$$
\begin{array}{cccccc}
\phi_{k l i} \phi_{m l i} & \phi_{k l l} \phi_{m i i} & \phi_{k m l} \phi_{l i i} & \phi_{m l i} \phi_{k l i} & \phi_{m i i} \phi_{k l l} & \phi_{l i i} \phi_{k m l} \\
\phi_{k l l} \phi_{m l l} & \phi_{k m l} \phi_{l l l} & \phi_{m l l} \phi_{k l l} & \phi_{l l l} \phi_{k m l} &
\end{array}
$$

where $l$ and $i$ are modes diagonal overall and the order indicates the order of the bra-kets in Equation (49).

In the 1 and 3 and in the 3 and 1 cases, if two other modes are considered, the overall matrix element is zero since each other mode would have $q_{i}$ of odd degree. Nonzero matrix elements are possible if one other mode is considered, and the associated force constants of these matrix elements are

$$
\begin{array}{cccccc}
\phi_{k l l} \phi_{m m m} & \phi_{k m l} \phi_{m m l} & \phi_{k m m} \phi_{m l l} & \phi_{m m m} \phi_{k l l} & \phi_{m m l} \phi_{k m l} & \phi_{m l l} \phi_{k m m} \\
\phi_{m l l} \phi_{k k k} & \phi_{m k l} \phi_{k k l} & \phi_{m k k} \phi_{k l l} & \phi_{k k k} \phi_{m l l} & \phi_{k k l} \phi_{m k l} & \phi_{k l l} \phi_{m k k}
\end{array}
$$

In the 3 and 3 case, there are two possible classes of matrix elements, and their associated force constants are

$$
\phi_{k k k} \phi_{m m m} \quad \phi_{k k m} \phi_{m m k} \quad \phi_{m m m} \phi_{k k k} \quad \phi_{m m k} \phi_{k k m}
$$

In the 1 and 5 and in the 5 and 1 cases, there clearly is one possible matrix element type for each, with the associated force constants

$$
\begin{array}{ll}
\phi_{k m m} \phi_{m m m} & \phi_{m m m} \phi_{k m m} \\
\phi_{k k k} \phi_{k k m} & \phi_{k k m} \phi_{k k k}
\end{array}
$$

Within these sets of force constants, there are many possible nonzero matrix elements. Due to their large number they are not presented here. Some of them combine to give zero contribution to the 1-1 resonance matrix element due to cancellation of terms. The force constants for terms that cancel are

$$
\begin{aligned}
& \phi_{m l l} \phi_{k k k} \quad \phi_{k k k} \phi_{m l l} \\
& \phi_{k k k} \phi_{m m m} \quad \phi_{m m m} \phi_{k k k}
\end{aligned}
$$




$$
\begin{array}{cc}
\phi_{k l l} \phi_{m i i} & \\
\phi_{k l l} \phi_{m m m} & \phi_{m i i} \phi_{k l l} \phi_{k l l} \\
\phi_{m l l} \phi_{k k k} & \phi_{k k k} \phi_{m l l}
\end{array}
$$

This leaves twenty of thirty matrix element types to check, which still is a laborious task. Within a force constant pair, the terms are grouped according to their energy denominators, and the expression is simplified algebraically. When all of the second order cubic matrix elements are considered, they can be grouped into three general cases, with the quantum number dependences

$$
\begin{aligned}
& \frac{\sqrt{\left(n_{k}+1\right)\left(n_{m}+1\right)}}{4}\left(n_{k}+1\right) \\
& \frac{\sqrt{\left(n_{k}+1\right)\left(n_{m}+1\right)}}{4}\left(n_{m}+1\right) \\
& \frac{\sqrt{\left(n_{k}+1\right)\left(n_{m}+1\right)}}{2}\left(n_{l}+\frac{1}{2}\right)
\end{aligned}
$$

so that the contributing second order cubic matrix elements for a 1-1 resonance become 


$$
\begin{aligned}
& \frac{1}{2} \sum_{\gamma}\left\langle n_{k}+1, n_{m}, n_{l}\left|h c \sum_{k l m} \frac{1}{3 !} \phi_{k l m} q_{k} q_{l} q_{m}\right| \gamma\right\rangle\left\langle\gamma\left|h c \sum_{k^{\prime} l^{\prime} m^{\prime}} \frac{1}{3 !} \phi_{k^{\prime} l^{\prime} m^{\prime}} q_{k^{\prime}} q_{l^{\prime}} q_{m^{\prime}}\right| n_{k}, n_{m}+1, n_{l}\right\rangle\left(\frac{1}{E_{a}^{o}-E_{\gamma}^{o}}+\frac{1}{E_{b}^{o}-E_{\gamma}^{o}}\right) \\
& =\frac{\sqrt{\left(n_{k}+1\right)\left(n_{m}+1\right)}}{4}\left(n_{k}+1\right) \cdot\left\{-h c \frac{1}{8} \sum_{i} \phi_{k m i} \phi_{k k i}\left[\frac{1}{2 \omega_{k}+\omega_{i}}+\frac{1}{-2 \omega_{k}+\omega_{i}}\right.\right. \\
& \left.\left.+\frac{4}{\omega_{i}}+\frac{2}{\omega_{m}-\omega_{k}+\omega_{i}}+\frac{2}{-\omega_{m}+\omega_{k}+\omega_{i}}+\frac{1}{\omega_{m}+\omega_{k}+\omega_{i}}+\frac{1}{-\omega_{m}-\omega_{k}+\omega_{i}}\right]\right\} \\
& +\frac{\sqrt{\left(n_{k}+1\right)\left(n_{m}+1\right)}}{4}\left(n_{m}+1\right) \cdot\left\{-h c \frac{1}{8} \sum_{i} \phi_{k m i} \phi_{m m i}\left[\frac{1}{2 \omega_{m}+\omega_{i}}+\frac{1}{-2 \omega_{m}+\omega_{i}}\right.\right. \\
& \left.\left.+\frac{4}{\omega_{i}}+\frac{2}{\omega_{k}-\omega_{m}+\omega_{i}}+\frac{2}{-\omega_{k}+\omega_{m}+\omega_{i}}+\frac{1}{\omega_{k}+\omega_{m}+\omega_{i}}+\frac{1}{-\omega_{k}-\omega_{m}+\omega_{i}}\right]\right\} \\
& +\frac{\sqrt{\left(n_{k}+1\right)\left(n_{m}+1\right)}}{2} \sum_{l \neq k, m}\left(n_{l}+\frac{1}{2}\right) \cdot\left\{-h c \frac{1}{8} \sum_{i} \phi_{k l i} \phi_{m l i}\left[\frac{1}{\omega_{k}+\omega_{l}+\omega_{i}}+\frac{1}{-\omega_{k}-\omega_{l}+\omega_{i}}+\frac{1}{\omega_{m}+\omega_{l}+\omega_{i}}\right.\right. \\
& \left.+\frac{1}{-\omega_{m}-\omega_{l}+\omega_{i}}+\frac{1}{\omega_{k}-\omega_{l}+\omega_{i}}+\frac{1}{-\omega_{k}+\omega_{l}+\omega_{i}}+\frac{1}{-\omega_{m}+\omega_{l}+\omega_{i}}+\frac{1}{\omega_{m}-\omega_{l}+\omega_{i}}\right] \\
& \left.-h c \frac{1}{8} \sum_{i} \phi_{k m i} \phi_{l i i}\left[\frac{1}{\omega_{k}-\omega_{m}+\omega_{i}}+\frac{1}{-\omega_{k}+\omega_{m}+\omega_{i}}+\frac{2}{\omega_{i}}\right]\right\}
\end{aligned}
$$

where $a$ and $b$ are the class 1 states in resonance and $\gamma$ is a connecting class 2 state.

Note that the quartic, vibrational angular momentum, and second order cubic matrix elements for the 1-1 resonance have the same three quantum number dependences. The matrix elements therefore can be combined to give the total 1-1 resonance matrix element 


$$
\begin{aligned}
& \left\langle n_{k}+1, n_{m}, n_{l}|\tilde{H}| n_{k}, n_{m}+1, n_{l}\right\rangle \\
& =\frac{\sqrt{\left(n_{k}+1\right)\left(n_{m}+1\right)}}{4}\left(n_{k}+1\right)\left\{h c \frac{1}{2} \phi_{k k k m}-h c \frac{1}{8} \sum_{i} \phi_{m k i} \phi_{k k i}\left[\frac{1}{2 \omega_{k}+\omega_{i}}+\frac{1}{-2 \omega_{k}+\omega_{i}}\right.\right. \\
& \left.\left.+\frac{4}{\omega_{i}}+\frac{2}{\omega_{m}-\omega_{k}+\omega_{i}}+\frac{2}{-\omega_{m}+\omega_{k}+\omega_{i}}+\frac{1}{\omega_{m}+\omega_{k}+\omega_{i}}+\frac{1}{-\omega_{m}-\omega_{k}+\omega_{i}}\right]\right\} \\
& +\frac{\sqrt{\left(n_{k}+1\right)\left(n_{m}+1\right)}}{4}\left(n_{m}+1\right)\left\{h c \frac{1}{2} \phi_{k m m m}-h c \frac{1}{8} \sum_{i} \phi_{k m i} \phi_{m m i}\left[\frac{1}{2 \omega_{m}+\omega_{i}}+\frac{1}{-2 \omega_{m}+\omega_{i}}\right.\right. \\
& \left.\left.+\frac{4}{\omega_{i}}+\frac{2}{\omega_{k}-\omega_{m}+\omega_{i}}+\frac{2}{-\omega_{k}+\omega_{m}+\omega_{i}}+\frac{1}{\omega_{k}+\omega_{m}+\omega_{i}}+\frac{1}{-\omega_{k}-\omega_{m}+\omega_{i}}\right]\right\} \\
& +\frac{\sqrt{\left(n_{k}+1\right)\left(n_{m}+1\right)}}{2} \sum_{l \neq k, m}\left(n_{l}+\frac{1}{2}\right) \cdot\left\{h c \frac{1}{2} \phi_{k l l m}+2 \sum_{\alpha} B_{\alpha} \frac{\xi_{k l}^{\alpha} \xi_{m l}^{\alpha}\left(\omega_{l}^{2}+\omega_{k} \omega_{m}\right)}{\omega_{l}\left(\omega_{k} \omega_{m}\right)^{1 / 2}}\right. \\
& -h c \frac{1}{8} \sum_{i} \phi_{k l i} \phi_{m l i}\left[\frac{1}{\omega_{k}+\omega_{l}+\omega_{i}}+\frac{1}{-\omega_{k}-\omega_{l}+\omega_{i}}+\frac{1}{\omega_{m}+\omega_{l}+\omega_{i}}+\frac{1}{-\omega_{m}-\omega_{l}+\omega_{i}}\right. \\
& \left.+\frac{1}{\omega_{k}-\omega_{l}+\omega_{i}}+\frac{1}{-\omega_{k}+\omega_{l}+\omega_{i}}+\frac{1}{-\omega_{m}+\omega_{l}+\omega_{i}}+\frac{1}{\omega_{m}-\omega_{l}+\omega_{i}}\right] \\
& \left.-h c \frac{1}{8} \sum_{i} \phi_{k m i} \phi_{l l i}\left[\frac{1}{\omega_{k}-\omega_{m}+\omega_{i}}+\frac{1}{-\omega_{k}+\omega_{m}+\omega_{i}}+\frac{2}{\omega_{i}}\right]\right\} \\
& =\frac{\sqrt{\left(n_{k}+1\right)\left(n_{m}+1\right)}}{4}\left(n_{k}+1\right)\left\{h c \frac{1}{2} \phi_{k k k m}-h c \frac{1}{8} \sum_{i} \phi_{m k i} \phi_{k k i}\left[\frac{1}{2 \omega_{k}+\omega_{i}}+\frac{1}{-2 \omega_{k}+\omega_{i}}\right.\right. \\
& \left.\left.+\frac{4}{\omega_{i}}+\frac{2}{\omega_{m}-\omega_{k}+\omega_{i}}+\frac{2}{-\omega_{m}+\omega_{k}+\omega_{i}}+\frac{1}{\omega_{m}+\omega_{k}+\omega_{i}}+\frac{1}{-\omega_{m}-\omega_{k}+\omega_{i}}\right]\right\} \\
& +\left(n_{m}+1\right)\left\{h c \frac{1}{2} \phi_{k m m m}-h c \frac{1}{8} \sum_{i} \phi_{k m i} \phi_{m m i}\left[\frac{1}{2 \omega_{m}+\omega_{i}}+\frac{1}{-2 \omega_{m}+\omega_{i}}\right.\right. \\
& \left.\left.+\frac{4}{\omega_{i}}+\frac{2}{\omega_{k}-\omega_{m}+\omega_{i}}+\frac{2}{-\omega_{k}+\omega_{m}+\omega_{i}}+\frac{1}{\omega_{k}+\omega_{m}+\omega_{i}}+\frac{1}{-\omega_{k}-\omega_{m}+\omega_{i}}\right]\right\} \\
& +2 \sum_{l \neq k, m}\left(n_{l}+\frac{1}{2}\right) \cdot\left\{h c \frac{1}{2} \phi_{k l l m}+2 \sum_{\alpha} B_{\alpha} \frac{\zeta_{k l}^{\alpha} \zeta_{m l}^{\alpha}\left(\omega_{l}^{2}+\omega_{k} \omega_{m}\right)}{\omega_{l}\left(\omega_{k} \omega_{m}\right)^{1 / 2}}\right. \\
& -h c \frac{1}{8} \sum_{i} \phi_{k l i} \phi_{m l i}\left[\frac{1}{\omega_{k}+\omega_{l}+\omega_{i}}+\frac{1}{-\omega_{k}-\omega_{l}+\omega_{i}}+\frac{1}{\omega_{m}+\omega_{l}+\omega_{i}}+\frac{1}{-\omega_{m}-\omega_{l}+\omega_{i}}\right. \\
& \left.+\frac{1}{\omega_{k}-\omega_{l}+\omega_{i}}+\frac{1}{-\omega_{k}+\omega_{l}+\omega_{i}}+\frac{1}{-\omega_{m}+\omega_{l}+\omega_{i}}+\frac{1}{\omega_{m}-\omega_{l}+\omega_{i}}\right] \\
& \left.-h c \frac{1}{8} \sum_{i} \phi_{k m i} \phi_{l l i}\left[\frac{1}{\omega_{k}-\omega_{m}+\omega_{i}}+\frac{1}{-\omega_{k}+\omega_{m}+\omega_{i}}+\frac{2}{\omega_{i}}\right]\right\}
\end{aligned}
$$




$$
=\frac{\sqrt{\left(n_{k}+1\right)\left(n_{m}+1\right)}}{2}\left\{\frac{3}{2}\left(n_{k}+1\right) K_{k k ; m k}+\frac{3}{2}\left(n_{m}+1\right) K_{k m ; m m}+\sum_{l \neq k, m}\left(n_{l}+\frac{1}{2}\right) K_{k l ; m l}\right\}
$$

where

$$
\begin{gathered}
K_{k k ; m k}=h c \frac{1}{6} \phi_{k k k m}-h c \frac{1}{24} \sum_{i} \phi_{m k i} \phi_{k k i}\left[\frac{1}{2 \omega_{k}+\omega_{i}}+\frac{1}{-2 \omega_{k}+\omega_{i}}\right. \\
\left.+\frac{4}{\omega_{i}}+\frac{2}{\omega_{m}-\omega_{k}+\omega_{i}}+\frac{2}{-\omega_{m}+\omega_{k}+\omega_{i}}+\frac{1}{\omega_{m}+\omega_{k}+\omega_{i}}+\frac{1}{-\omega_{m}-\omega_{k}+\omega_{i}}\right] \\
\left.+\frac{4}{\omega_{i}}+\frac{219)}{K_{k m ; m m}-h c \frac{1}{6} \phi_{k m m m}-h c \frac{1}{24} \sum_{i} \phi_{k m i} \phi_{m m i}\left[\frac{1}{2 \omega_{m}+\omega_{i}}+\frac{1}{-2 \omega_{m}+\omega_{i}}\right.}+\frac{1}{-\omega_{k}+\omega_{m}+\omega_{i}}+\frac{1}{\omega_{k}+\omega_{m}+\omega_{i}}+\frac{1}{-\omega_{k}-\omega_{m}+\omega_{i}}\right] \\
K_{k l ; m l}=h c \frac{1}{2} \phi_{k l l m}+2 \sum_{\alpha} B_{\alpha} \frac{\zeta_{k l}^{\alpha} \zeta_{m l}^{\alpha}\left(\omega_{l}^{2}+\omega_{k} \omega_{m}\right)}{\omega_{l}\left(\omega_{k} \omega_{m}\right)^{1 / 2}} \\
-h c \frac{1}{8} \sum_{i} \phi_{k l i} \phi_{m l i}\left[\frac{1}{\omega_{k}+\omega_{l}+\omega_{i}}+\frac{1}{-\omega_{k}-\omega_{l}+\omega_{i}}+\frac{1}{\omega_{m}+\omega_{l}+\omega_{i}}+\frac{1}{-\omega_{m}-\omega_{l}+\omega_{i}}\right. \\
\left.+\frac{1}{\omega_{k}-\omega_{l}+\omega_{i}}+\frac{1}{-\omega_{k}+\omega_{l}+\omega_{i}}+\frac{1}{-\omega_{m}+\omega_{l}+\omega_{i}}+\frac{1}{\omega_{m}-\omega_{l}+\omega_{i}}\right] \\
\sum_{i} \phi_{k m i} \phi_{l l i}\left[\frac{1}{\omega_{k}-\omega_{m}+\omega_{i}}+\frac{2}{-\omega_{k}+\omega_{m}+\omega_{i}}+\frac{1}{\omega_{i}}\right]
\end{gathered}
$$

Note that in contrast to other resonances which include a single resonance constant, the 1-1 resonance involves $3 N-6$ resonance constants. This makes fitting 1-1 resonances to experimental data difficult.

Equations (118)-(121) were verified numerically in Section VII and they correct numerous typographical errors in Lehmann's Equations (9) and (10) [3], as well as in Martin and 
Taylor's Equations (15) and (16) [4], and minor errors in Matthew et al.'s Equations (5) and (6) [6].

\section{Resonance denominators in diagonal matrix element, anharmonic constant, and resonance constant expressions}

In all of the expressions derived in IV.A. and IV.B, the notation does not indicate which states are omitted from the sum over states. Equation (49) is a sum over class 2 states $\gamma$ that does not include the strongly interacting class 1 states, and the left-hand sides of the equations following Equation (49) in IV.A. and IV.B also exclude class 1 states. Nevertheless, the right-hand sides do not explicitly indicate which terms are omitted. The sums over modes include all modes, but not all states are included when the expressions are used. Strongly interacting class 1 states must be excluded from the sum over all class 2 states. If these states were included, then the result would be inaccurate due to the additional large terms from the resonances. The following section elaborates on this concept.

\section{Discussion}

\section{A. Effect of resonances on anharmonic constants}

Van Vleck perturbation theory simultaneously accounts for strong interactions between states in resonance as well as weak interactions with other states. Resonances cause large discrepancies in values calculated using second order perturbation theory (PT2), and consequently considering their effects is of great importance. Examples of such resonances include the Fermi resonance in carbon dioxide [29] and the Darling-Dennison resonance in water [30].

In principle, treating resonances involves including their effect in the class 1 matrix and then excluding the resonant class 1 states from the summation over class 2 states in Equation (49). If a resonance in the summation over $\gamma$ is present, it can cause very small energy denominators (and hence very large individual terms) due to the near degeneracy of 
Fermi resonances, $\omega_{k} \approx \omega_{l}+\omega_{m}$. As will be seen, examples of denominators where such resonances become problematic include $\omega_{k}-\omega_{l}-\omega_{m}$ and $-\omega_{k}+\omega_{l}+\omega_{m}$. In practice the exclusion of class 1 states from the summation over $\gamma$ is accomplished by the exclusion of terms with resonant denominators.

In the case of diagonal matrix elements, terms with such energy denominators are present in the final results, as shown in Equation (63). Thus, in order to exclude resonance denominators, partial fraction expansion must be performed in order to isolate the resonance denominators. Anharmonic constants which include removal of resonant denominators are usually marked with an asterisk, e.g., $x_{k l}^{*}$. The exclusion of resonant denominators to form anharmonic constants $x_{k k}^{*}$ and $x_{k l}^{*}$ has been discussed by Califano as well as by Papoušek and Aliev [1-2].

There are seven terms in Equation (65) where resonance denominators are present: the first, fifth, sixth, seventh, eighth, ninth, and tenth terms. When partial fraction decomposition has been performed on each term, Equation (65) becomes

$h^{2} c^{2} \sum_{\gamma \neq a} \frac{\left\langle a\left|\sum_{k l m} \frac{1}{3 !} \phi_{k l m} q_{k} q_{l} q_{m}\right| \gamma\right\rangle\langle\gamma| \sum_{k^{\prime} l^{\prime} m^{3}} 3 !}{\frac{1}{3 !} \phi_{k^{\prime} l^{\prime} m^{\prime}} q_{k^{\prime}} q_{l^{\prime}} q_{m^{\prime}}|a\rangle}=$ 


$$
\begin{aligned}
& -h c \sum_{k \neq l} \frac{1}{16} \phi_{k k l}^{2}\left[\frac{2}{\omega_{l}}+\frac{1}{2\left(2 \omega_{k}+\omega_{l}\right)}-\frac{1}{2\left(2 \omega_{k}-\omega_{l}\right)}\right]\left(v_{k}+\frac{1}{2}\right)^{2}-h c \sum_{k} \frac{5}{48} \phi_{k k k}^{2} \frac{1}{\omega_{k}}\left(v_{k}+\frac{1}{2}\right)^{2} \\
& -h c \sum_{k \neq l \neq m} \frac{1}{8} \phi_{k k m} \phi_{l l m} \frac{1}{\omega_{m}}\left(v_{k}+\frac{1}{2}\right)\left(v_{l}+\frac{1}{2}\right)-h c \sum_{k \neq l} \frac{1}{4} \phi_{k k k} \phi_{k l l} \frac{1}{\omega_{k}}\left(v_{k}+\frac{1}{2}\right)\left(v_{l}+\frac{1}{2}\right) \\
& -h c \sum_{k \neq l \neq m} \frac{\phi_{k l m}^{2}}{12}\left[\frac{1}{4\left(\omega_{k}+\omega_{l}+\omega_{m}\right)}+\frac{1}{4\left(-\omega_{k}-\omega_{l}+\omega_{m}\right)}+\frac{1}{4\left(\omega_{k}-\omega_{l}+\omega_{m}\right)}+\frac{1}{4\left(-\omega_{k}+\omega_{l}+\omega_{m}\right)}\right]\left(\mathrm{v}_{k}+\frac{1}{2}\right)\left(\mathrm{v}_{l}+\frac{1}{2}\right) \\
& -h c \sum_{k \neq l \neq m} \frac{\phi_{k l m}^{2}}{12}\left[\frac{1}{4\left(\omega_{k}+\omega_{l}+\omega_{m}\right)}-\frac{1}{4\left(-\omega_{k}-\omega_{l}+\omega_{m}\right)}-\frac{1}{4\left(\omega_{k}-\omega_{l}+\omega_{m}\right)}+\frac{1}{4\left(-\omega_{k}+\omega_{l}+\omega_{m}\right)}\right]\left(\mathrm{v}_{k}+\frac{1}{2}\right)\left(\mathrm{v}_{m}+\frac{1}{2},\right. \\
& -h c \sum_{k \neq l \neq m} \frac{\phi_{k l m}^{2}}{12}\left[\frac{1}{4\left(\omega_{k}+\omega_{l}+\omega_{m}\right)}-\frac{1}{4\left(-\omega_{k}-\omega_{l}+\omega_{m}\right)}+\frac{1}{4\left(\omega_{k}-\omega_{l}+\omega_{m}\right)}-\frac{1}{4\left(-\omega_{k}+\omega_{l}+\omega_{m}\right)}\right]\left(\mathrm{v}_{l}+\frac{1}{2}\right)\left(\mathrm{v}_{m}+\frac{1}{2},\right. \\
& -h c \sum_{k \neq l} \frac{\phi_{k k l}^{2}}{2}\left[\frac{1}{4\left(2 \omega_{k}+\omega_{l}\right)}+\frac{1}{4\left(2 \omega_{k}-\omega_{l}\right)}\right]\left(\mathrm{v}_{k}+\frac{1}{2}\right)\left(\mathrm{v}_{l}+\frac{1}{2}\right) \\
& -h c \sum_{k \neq l \neq m} \frac{\phi_{k l m}^{2}}{24}\left[\frac{1}{8\left(\omega_{k}+\omega_{l}+\omega_{m}\right)}+\frac{1}{8\left(-\omega_{k}-\omega_{l}+\omega_{m}\right)}-\frac{1}{8\left(\omega_{k}-\omega_{l}+\omega_{m}\right)}-\frac{1}{8\left(-\omega_{k}+\omega_{l}+\omega_{m}\right)}\right] \\
& +h c \sum_{k \neq l} \frac{3}{64} \phi_{k k l}^{2}\left[-\frac{1}{2\left(2 \omega_{k}+\omega_{l}\right)}+\frac{1}{2\left(2 \omega_{k}-\omega_{l}\right)}\right]-h c \sum_{k} \frac{7}{576} \phi_{k k k}^{2}
\end{aligned}
$$

Similarly, once partial fraction decomposition has been performed on the terms in $x_{k k}$ and $x_{k l}$ with resonance denominators, Equations (78) and (80) become, respectively,

$$
\begin{gathered}
x_{k k}=h c \frac{1}{16} \phi_{k k k k}-h c \sum_{l} \frac{1}{16} \phi_{k k l}^{2}\left[\frac{2}{\omega_{l}}+\frac{1}{2\left(2 \omega_{k}+\omega_{l}\right)}-\frac{1}{2\left(2 \omega_{k}-\omega_{l}\right)}\right] \\
x_{k l}=h c \frac{1}{4} \phi_{k k l l}-h c \sum_{m} \frac{\phi_{k l m}^{2}}{2}\left[\frac{1}{4\left(\omega_{k}+\omega_{l}+\omega_{m}\right)}+\frac{1}{4\left(\omega_{k}+\omega_{l}-\omega_{m}\right)}+\frac{1}{4\left(\omega_{k}-\omega_{l}+\omega_{m}\right)}+\frac{1}{4\left(\omega_{k}-\omega_{l}-\omega_{m}\right)}\right] \\
-h c \sum_{m} \phi_{k k m} \phi_{l l m} \frac{1}{4 \omega_{m}}+\sum_{\alpha} B_{\alpha}\left(\zeta_{k l}^{\alpha}\right)^{2}\left(\frac{\omega_{l}}{\omega_{k}}+\frac{\omega_{k}}{\omega_{l}}\right)
\end{gathered}
$$

A convenient notation for the denominators expresses the resonance denominators as $[4,31]$

$$
\frac{1}{\left( \pm \omega_{k} \pm \omega_{l} \pm \omega_{m}\right)}=D( \pm k, \pm l, \pm m)
$$

For example, $D(k,-l, m)=\frac{1}{\left(\omega_{k}-\omega_{l}+\omega_{m}\right)}$. 
Equation (122) then becomes

$$
\begin{aligned}
& h^{2} c^{2} \sum_{\gamma \neq a} \frac{\left\langle a\left|\sum_{k l m} \frac{1}{3 !} \phi_{k l m} q_{k} q_{l} q_{m}\right| \gamma\right\rangle\left\langle\gamma\left|\sum_{k^{\prime} l^{\prime} m^{3}} \frac{1}{3 !} \phi_{k^{\prime} l^{\prime} m^{\prime}} q_{k^{\prime}} q_{l^{\prime}} q_{m^{\prime}}\right| a\right\rangle}{E_{a}^{o}-E_{\gamma}^{o}}= \\
& -h c \sum_{k \neq l} \frac{1}{16} \phi_{k k l}^{2}\left[\frac{2}{\omega_{l}}+\frac{1}{2} D(k, k, l)-\frac{1}{2} D(k, k,-l)\right]\left(\mathrm{v}_{k}+\frac{1}{2}\right)^{2}-h c \sum_{k} \frac{5}{48} \phi_{k k k}^{2} \frac{1}{\omega_{k}}\left(\mathrm{v}_{k}+\frac{1}{2}\right)^{2} \\
& -h c \sum_{k \neq l \neq m} \frac{1}{8} \phi_{k k m} \phi_{l l m} \frac{1}{\omega_{m}}\left(\mathrm{v}_{k}+\frac{1}{2}\right)\left(\mathrm{v}_{l}+\frac{1}{2}\right)-h c \sum_{k \neq l} \frac{1}{4} \phi_{k k k} \phi_{k l l} \frac{1}{\omega_{k}}\left(\mathrm{v}_{k}+\frac{1}{2}\right)\left(\mathrm{v}_{l}+\frac{1}{2}\right) \\
& -h c \sum_{k \neq l \neq m} \frac{\phi_{k l m}^{2}}{12}\left[\frac{1}{4} D(k, l, m)+\frac{1}{4} D(-k,-l, m)+\frac{1}{4} D(k,-l, m)+\frac{1}{4} D(-k, l, m)\right]\left(\mathrm{v}_{k}+\frac{1}{2}\right)\left(\mathrm{v}_{l}+\frac{1}{2}\right) \\
& -h c \sum_{k \neq l \neq m} \frac{\phi_{k l m}^{2}}{12}\left[\frac{1}{4} D(k, l, m)-\frac{1}{4} D(-k,-l, m)-\frac{1}{4} D(k,-l, m)+\frac{1}{4} D(-k, l, m)\right]\left(\mathrm{v}_{k}+\frac{1}{2}\right)\left(\mathrm{v}_{m}+\frac{1}{2}\right) \\
& -h c \sum_{k \neq l \neq m} \frac{\phi_{k l m}^{2}}{12}\left[\frac{1}{4} D(k, l, m)-\frac{1}{4} D(-k,-l, m)+\frac{1}{4} D(k,-l, m)-\frac{1}{4} D(-k, l, m)\right]\left(\mathrm{v}_{l}+\frac{1}{2}\right)\left(\mathrm{v}_{m}+\frac{1}{2}\right) \\
& -h c \sum_{k \neq l} \frac{\phi_{k k l}^{2}}{2}\left[\frac{1}{4} D(k, k, l)+\frac{1}{4} D(k, k,-l)\right]\left(\mathrm{v}_{k}+\frac{1}{2}\right)\left(\mathrm{v}_{l}+\frac{1}{2}\right) \\
& -h c \sum_{k \neq l \neq m} \frac{\phi_{k l m}^{2}}{24}\left[\frac{1}{8} D(k, l, m)+\frac{1}{8} D(-k,-l, m)-\frac{1}{8} D(k,-l, m)-\frac{1}{8} D(-k, l, m)\right] \\
& +h c \sum_{k \neq l} \frac{3}{64} \phi_{k k l}^{2}\left[-\frac{1}{2} D(k, k, l)+\frac{1}{2} D(k, k,-l)\right]-h c \sum_{k} \frac{7}{576} \phi_{k k k}^{2} \\
& -h(
\end{aligned}
$$

The anharmonic constants expressed using D notation are presented in Tables 2 and 3.

\section{B. Effect of resonances on resonance constants}

The resonance constants of Section IV.B are expressed in terms of the "D" notation quite easily, as they naturally occur in terms of partial fractions, which facilitate treating the effects of resonances. Lehmann, Law, Matthews et al., and Hänninen and Halonen do not expand their expressions into partial fractions and therefore do not allow for the effect of resonances on the anharmonic and resonance constants to be conveniently treated in their formulation $[3,5,6,28]$. The D expressions for resonance constants are presented in Tables 2 and 3. These tables, along with a set of resonances, completely define the computational 
method VPT2+K. The matrix elements in Tables 2 and 3 are carefully constructed as a constant multiplied by a harmonic oscillator matrix element, with the constant written in terms of partial fractions to facilitate fitting and the removal of class 1 states from the summation, i.e., the exclusion of resonance denominators.

\section{The exclusion of class 1 states from the sum over intermediate states is equivalent to the exclusion of resonant denominators}

If a Fermi resonance is present among specific modes $k$ and $l$ and $m$, D's of the form $D(-k, l, m), D(k,-l,-m), D(l,-k, m)$, etc. become extremely large since $\omega_{k} \approx \omega_{l}+\omega_{m}$. Consequently they are not adequately treated by perturbation theory, and their inclusion would lead to highly inaccurate results.

Setting the resonant $\mathrm{D}$ terms in Table 3 equal to zero is equivalent to excluding resonant states from the second order cubic summations, i.e., the summation over intermediate states $\gamma$, in Equation (49). The resonant states are strongly interacting class 1 states, and as such they are no longer considered class 2 states included in the summation over $\gamma$. For any two states, the energy difference between them indicates whether or not they are in resonance. If the two states are in resonance, the energy difference will be very small. Since $E=h c \omega$, the energy difference is characterized by the vibrational frequency difference between the two states. Resonance denominators arise from Fermi resonances, e.g., $\omega_{k} \approx \omega_{l}+\omega_{m}$, so resonance denominators have the form $\omega_{k}-\omega_{l}-\omega_{m}$ or $-\omega_{k}+\omega_{l}+\omega_{m}$. Then when two states are in resonance, the energy denominators in Equation (40) become $h c\left(\omega_{k}-\omega_{l}-\omega_{m}\right)$ or $h c\left(-\omega_{k}+\omega_{l}+\omega_{m}\right)$. The terms in the equations from IV with denominators other than those resulting from Fermi resonances arise from other possible intermediate states. As seen in Equation (40), each intermediate state gives rise to only two energy denominators. For states in Fermi resonance, the only terms present are those two 
with resonance denominators. Interacting class 1 states thereby give rise to a resonant denominator. When terms with resonant denominators due to a particular resonance are set to zero, the effect of that resonance is removed, and its exclusion prevents the consideration of an interaction caused by that resonance and thereby excludes the resonant state from the summation. Thus the partial fraction-expanded expressions are crucial for the practical implementation of the Van Vleck transformation and the attainment of results comparable to experimentally observed spectroscopic constants.

The computer programs encoding the algebraic VVPT results utilize Equation (126) as well as the expressions in Table 3, enabling resonances to be treated explicitly. Further discussion of the code is presented in Section VI. Spectroscopic constants computed according to the expressions in Table 3 are presented for a few example molecules in Section VII and in Davisson et al. [31].

\section{Effect of resonances on zero-point energy}

Although removing terms with resonance denominators allows for more accurate treatment of resonances, this procedure affects the calculated zero-point energy since resonance denominators are present in the constant terms in Equation (77) and in the zeropoint terms arising from the terms with quantum number dependences in Equation (74). When resonances are accounted for in vibrational energy calculations, they must be accounted for in the zero-point energy as well by removing terms with resonant denominators. Proper accounting of zero-point energy is necessary when determining vibrational energies relative to the energy minima computed by electronic structure calculations.

Schuurman et al. give expressions that exclude terms with resonant denominators that are consistent with Equations (122)-(124) with the exception of their $Z_{\text {kinetic }}$ term, which 
includes a contribution from the ignored constant in Equation (2) as well as an error in the vibrational angular momentum contribution [32].

\section{E. Incorporating VPT2+K into computational chemistry software}

The computational process for predicting vibrational energy levels begins by optimizing the geometry, computing the quartic force field, and performing a normal mode analysis to determine the constants $\omega_{i}, B_{\alpha}, \zeta, \phi_{k l m}$, and $\phi_{k l m n}$. Fermi (1-2) and DarlingDennison (1-1, 2-2, 1-3) resonances are identified among vibrational modes. The anharmonic constants $x$ and the appropriate resonance constants $K$ then may be calculated, making certain to zero out the appropriate D's. Polyad matrices of resonantly interacting states are constructed based on the resonances, matrix elements of the transformed Hamiltonian are calculated using Tables 2 and 3, and the resulting Hamiltonian is diagonalized to obtain vibrational energy levels.

The results in Tables 2 and 3 are also useful for fitting experimental data. After completing the arduous task of recording and assigning vibrational spectra, Fermi and Darling-Dennison resonances are identified. From these resonances polyad matrices or strongly interacting states are constructed. Then resonance constants, scaled by harmonic oscillator matrix elements, are fit to the experimental energies. Note that one must be careful in associating the appropriate polyad eigenvalue to an experimental energy level; see Polik and van Ommen for one such automatic procedure for this process [26].

The form of matrix elements presented in Tables 2 and 3 is a constant multiplied by a harmonic oscillator matrix element, with the constant written in terms of partial fractions. This very practical form allows for generalized fitting of the constants to experimental data and easy removal of class 1 states from the summation by exclusion of terms with resonance denominators. 
Since the expressions presented here are general, in principle they can be applied to molecular systems of any size. It should be noted that, as with any other calculation, increasing the number of vibrational modes leads to significantly longer computation time. However, the VPT2+K calculation takes considerably less time than the determination of the underlying force field.

The VPT2+K code for vibrational energy matrix element expressions can be readily incorporated into quantum chemistry codes for more accurate calculation of vibrational energy levels from electronic structure calculations. Computational spectroscopy primarily focuses on the zero-point energies and fundamental frequencies and tends not to consider highly excited vibrational levels, particularly strongly interacting sets of levels in polyads. Currently, Gaussian09 can compute anharmonic constants $x_{k k}$ and $x_{k l}$, and it accounts for resonances with a reduced-dimensional variational approach [33-34]. This approach has been extended to account for resonance effects by a scheme that switches between PT2 and a degeneracy-corrected second order perturbation theory, in which nearly degenerate terms are rewritten to exclude resonance denominators and the effect of the resonance is estimated [35]. Another approach, the TOSH method, which is a first order treatment of shifted Hermite functions, does not encounter the difficulties associated with resonance denominators since it does not use second order perturbation theory [36]. CFOUR allows for manual inclusion of selected resonances [37]. Given the large effects of resonances on excited vibrational energy levels [26, 31, 38, 39], it is crucial that their effect be incorporated into such calculations. Future work needs to focus on determining criteria for identifying resonances $[7,31]$ and automatically including them into vibrational energy calculations.

\section{Computer implementation and verification}

In order to validate the accuracy and practicality of the derived formulae, the general expressions before algebraic manipulation and the matrix elements and resonance constants 
after algebraic manipulation were encoded into computer programs. The code was written in FORTRAN and compiled using both g77 and gfortran compilers to ensure generality and portability [40].

The verification presented in this section ensures that each step of the derivation process, to each of which there corresponds a computer program, is consistent with the previous step. Each program outputs the individual contributions to the vibrational energy and the total vibrational energy. Additionally, each program diagonalizes the Hamiltonian matrix and provides the eigenvalues along with their associated eigenvectors. The code for each program is given in the supplemental material.

The first program entitled HOME (short for "Harmonic Oscillator Matrix Elements") represents the most general form of both the on-diagonal and off-diagonal matrix element equations, essentially implementing Equation (40). The summations used throughout are nonrestrictive, accounting for all possible permutations of vibrational modes. The program reads in a block of class 1 states and the ab initio constants $\omega_{i}, B_{\alpha}, \zeta, \phi_{k l m}$, and $\phi_{k l m n}$. It finds valid intermediate states $\gamma$ using differences in quantum numbers between states. For the first order, second order quartic, and second order cubic contributions, the vibrational terms are computed explicitly using harmonic oscillator basis function integrals.

The second program, called E (short for "Energy"), encodes the algebraic expressions for vibrational energy levels given as Equation (126) and the expressions in Tables 2 and 3. Similar to HOME, E reads in a block of class 1 states and the ab initio constants $\omega_{i}, B_{\alpha}$, $\zeta, \phi_{k l m}$, and $\phi_{k l m n}$, though $\mathrm{E}$ also reads in the Fermi resonances in the molecule. The resonance denominators are excluded by pre-computing a D array and zeroing out all elements corresponding to the Fermi resonances present $[4,31]$. It determines whether each pair of class 1 states $a$ and $b$ gives a diagonal or off-diagonal matrix element and proceeds to 
calculate the matrix element. If the matrix element is off-diagonal, then it identifies the resonance if present, calculates the corresponding resonance constants, and computes the matrix element. E calculates the harmonic, first order, and second order contributions for both diagonal and off-diagonal matrix elements.

The third program is XKL (short for " $x_{k l}$ ”), which finds diagonal matrix elements by calculating the spectroscopically observable constants $\omega_{i}, x_{i i}, x_{i j}$, and computes off-diagonal matrix elements using the spectroscopic constants $K$. It implements Equations (123) and (124), which neglect non- $\left(v+\frac{1}{2}\right)$ terms, as well as the other expressions in Tables 2 and 3. All summations are non-restrictive. As with the previous program, XKL reads in a block of class 1 states, the $a b$ initio constants $\omega_{i}, B_{\alpha}, \zeta, \phi_{k l m}$, and $\phi_{k l m n}$, and the Fermi resonances. The resonance denominators are excluded by pre-computing a D array and zeroing out all elements corresponding to the Fermi resonances present $[4,31]$. Like E, it identifies whether each pair of states $a$ and $b$ gives a diagonal or off-diagonal matrix element and then calculates the matrix element. If the matrix element is off-diagonal, XKL identifies the resonance. The program then finds and uses the appropriate expression to compute the matrix element. After these calculations are performed, the neglected terms are added in using Equation (77) to account for dropped constant term $C$.

These three programs were used to calculate energy levels for quantum states of formaldehyde. To validate the calculated on-diagonal matrix elements, the results for the $\langle 11111|$ state without resonances are shown in Table 4. The quartic force field was computed at the CCSD(T)/ aug-cc-p-pVQZ level of theory by Davisson [31], and the $a b$ initio constants $\omega_{i}, B_{\alpha}, \zeta, \phi_{k l m}$, and $\phi_{k l m n}$ were calculated with SPECTRO [41]. Since each 
program represents a different step in the derivation process, the expressions are essentially equivalent forms and so should give identical results.

The off-diagonal matrix element expressions were validated by comparing the results of HOME and E for three off-diagonal matrix elements in formaldehyde with the polyad, or group of strongly interacting class 1 states, $\langle 020200|,\langle 020002|,\langle 010011|,\langle 000020|,\langle 001011|,\langle 011002|,\langle 011200|,\langle 002200|,\langle 002002|$, $\langle 200000|$. This calculation tested the formulae for $K_{k ; l m}\left(K_{3 ; 56}\right), K_{k k ; l l}\left(K_{44 ; 66}\right)$, and $K_{k l ; m l}\left(K_{2 ; 3}\right)$. The results are presented in Table 5.

As seen in Tables 4 and 5, though the different programs calculate matrix elements in distinct ways, they give identical final results. Additional comparisons were made to test each type of resonance. This validates the derivation process and insures the equivalence of the approaches for calculating vibrational energy levels.

\section{Examples}

Given an experimental data set in which the energy is written as a function of vibrational quantum numbers as in Equation (74), the data can be fit to the anharmonic constants $x_{k k}$ and $x_{k l}$ via overtones and a complete set of combination bands, respectively [31]. To fit to resonance constants, ideally there is enough data so that the problem is over-determined. Then linear least squares fitting can be used to determine the constants. In order to fit $K$ constants, resonances must be present in the polyad of interest, and appropriate quantum numbers are needed to numerically determine the constants through fitting.

The transformed vibrational Hamiltonian was computed for selected polyads of the isotopic set $\mathrm{H}_{2} \mathrm{CO}, \mathrm{HDCO}$, and $\mathrm{D}_{2} \mathrm{CO}$. Though small molecules, they contain resonances and thus serve as ideal examples for illustrating the value of VVPT in vibrational energy level analysis. Furthermore, since they are isotopomers of each other, they have the same potential 
but exhibit different resonances due to differences in atomic masses and molecular symmetry. For each molecule, the vibrational energy levels of a polyad of strongly interacting states will be presented as measured by experiment and as calculated by both PT2 and VVPT.

The ab initio constants $\omega_{i}, B_{\alpha}, \zeta, \phi_{k l m}$, and $\phi_{k l m n}$ were determined at the $\operatorname{CCSD(T)/aug-cc-p-~}$ pVQZ level of theory by Davisson [31], and the experimental data used in the comparison were obtained by dispersed fluorescence spectroscopy [26, 42-45]. All vibrational energies are reported relative to the zero-point energy.

\section{A. $\mathrm{H}_{2} \mathrm{CO}$}

The Fermi resonances present are $K_{5 ; 26}$ and $K_{5 ; 36}$, and the other resonances considered are $K_{44 ; 66}, K_{11 ; 55}$, and $K_{2 ; 3}$. Consider the ten-state polyad in Table 6 . Every vibrational state has been observed experimentally by Bouwens et al. and Ellsworth et al. [42, 44]. Ignoring the effects of resonances (PT2) results in a RMS error of $120 \mathrm{~cm}^{-1}$, whereas accounting for resonances $(\mathrm{VPT} 2+\mathrm{K})$ reduces the error by a factor of six to $18 \mathrm{~cm}^{-1}$.

The 1-1 resonance constant $K_{2 ; 3}$ between modes 2 and 3 was fit by Davisson as $K_{25 ; 35}$ [31]. In reality, this 1-1 resonance appears not as one resonance constant, but as a family of $3 N-6$ resonance constants $K_{2 i, 3 i}$, where $i$ represents one of $3 N-6=6$ vibrational modes in $\mathrm{H}_{2} \mathrm{CO}$. Clearly this presents a problem for fitting experimental data, as a resonance might only be observed with a limited number of other vibrational levels, leading to only a limited number of constants being determinable. Some of these constants might be zero or small, and their overall sign may be either positive or negative, leading to partial cancellation. The variety of values that can occur in a family of 1-1 resonance constants is illustrated for the $K_{2 i ; 3 i}$ constants for $\mathrm{H}_{2} \mathrm{CO}$ in Table 7 .

To determine a specific 1-1 resonance constant $K_{2 i ; 3 i}$, the 2-3 resonance needs to occur in a series of $v_{i}$ quantum number levels. For this particular data set, the 2-3 resonance was observed in a series of $\mathrm{v}_{5}$ levels. Since the $K_{25 ; 35}$ was the largest constant for the 2-3 
resonance in Table 7, it is not entirely unreasonable for the constant to have been labeled as such, especially if the resonance was not resolvable in series of other quantum number levels.

\section{B. HDCO}

The Fermi resonances present are $K_{5 ; 33}, K_{1 ; 44}$, and $K_{1 ; 66}$, and the other resonance considered is $K_{44 ; 66}$. Consider the six-state polyad in Table 8 connected by these resonances. All the vibrational levels have been observed experimentally by Ellsworth et al. [43]. Ignoring the effects of resonances results in a RMS error of $142 \mathrm{~cm}^{-1}$, whereas accounting for resonances reduces the error by a factor of seven to $21 \mathrm{~cm}^{-1}$.

\section{C. $\mathrm{D}_{2} \mathrm{CO}$}

The Fermi resonances present are $K_{5 ; 36}, K_{1 ; 44}$, and $K_{1 ; 66}$, and the other resonance considered is $K_{44 ; 66}$. Consider the six-state polyad in Table 9 connected by these resonances. Only some of the vibrational levels have been experimentally observed [45]. The RMS errors for both PT2 and VPT2+K are comparable to each other and also are comparable to the VPT2 $+\mathrm{K}$ errors for $\mathrm{H}_{2} \mathrm{CO}$ and $\mathrm{HDCO}$. This suggests that resonances are not as important in $\mathrm{D}_{2} \mathrm{CO}$ as those in $\mathrm{H}_{2} \mathrm{CO}$ and HDCO. Indeed Burleigh and Sibert [46] have suggested that in contrast to $\mathrm{H}_{2} \mathrm{CO}$, resonances are not important in $\mathrm{D}_{2} \mathrm{CO}$ at these energies. This can be seen from the comparison of the frequency differences between resonant modes in $\mathrm{D}_{2} \mathrm{CO}$ to those for $\mathrm{H}_{2} \mathrm{CO}$ and $\mathrm{HDCO}$. All of the $\omega_{k}-\omega_{l}-\omega_{m}$ frequency differences for Fermi resonances in $\mathrm{D}_{2} \mathrm{CO}$ are over $100 \mathrm{~cm}^{-1}$, whereas both $\mathrm{H}_{2} \mathrm{CO}$ and $\mathrm{HDCO}$ have Fermi resonances with frequency differences as low as $37 \mathrm{~cm}^{-1}$.

\section{Conclusion}

Nearly resonant vibrational energy levels are ubiquitous in molecules, and therefore a general approach for treating them is crucial. Van Vleck perturbation theory (VVPT) provides a means to account simultaneously for small perturbative and large resonant interactions among vibrational states. Second order VVPT is used in conjunction with a 
quartic force field to derive general expressions for matrix elements of polyatomic molecules that account for resonances and can be efficiently implemented into computer code. The formulations presented in Tables 2 and 3 involve resonance constants $K$ multiplied by harmonic oscillator matrix elements, which are useful both for fitting experimental data and computation of vibrational energy levels. They are written in partial fraction form so that the effects of Fermi resonances can be identified and accounted for easily. The formulae define a versatile computational technique, VPT2+K, for determining harmonic, anharmonic and resonance constants, $\omega, x$, and $K$, and the calculated vibrational energy levels are comparable to experimental data. Though validated using data from small molecules, the method in principle is applicable to larger molecules. The technique can be readily incorporated into quantum chemistry programs.

\section{Acknowledgements}

We gratefully acknowledge John L. Davisson for his computation of molecular force fields and Dr. Robert W. Field and Dr. Peter R. Taylor for many insightful discussions. This work was supported by the Howard Hughes Medical Institute and by National Science Foundation grants CHE-0624602 and CHE-1039925.

\section{References}

[1] S. Califano, Vibrational States (Wiley, Bristol, 1976).

[2] D. Papoušek and M.R. Aliev, Molecular Vibrational-Rotational Spectra (Elsevier, Prague, 1982).

[3] K.K. Lehmann, Mol. Phys. 66 (6), 1129 (1989); 75 (3)(E) 739 (1992).

[4] J.M.L. Martin and P.R. Taylor, Spectrochim. Acta, Part A. 53, 1039 (1997).

[5] V. Hänninen and L. Halonen, Mol. Phys. 101 (18), 2907 (2003).

[6] D.A. Matthews, J. Vázquez, and J.F. Stanton, Mol. Phys. 105 (19-22), 2659 (2007).

[7] S.V. Krasnoshchekov, E.V. Isayeva, and N.F. Stepanov, J. Chem. Phys. A 116, 3691 (2012).

[8] J.K.G. Watson, Mol. Phys. 15 (5), 479 (1968). 
[9] H.H. Nielsen, Phys. Rev. 68 (7-8), 181 (1945).

[10] H.H. Nielsen in Handbuch der Physik, edited by S. Flügge (Springer-Verlag, Berlin, 1959).

[11] G. Amat, H.H. Nielsen, and G. Tarrago, Rotation-Vibration of Polyatomic Molecules (Dekker, New York, 1971).

[12] I.M. Mills, in Molecular Spectroscopy: Modern Research, vol. 1, edited by K.N. Rao (Academic Press, Orlando, 1972), pp. 115-140.

[13] M.R. Aliev and J.K.G. Watson, in Molecular Spectroscopy: Modern Research, vol. 3, edited by K.N. Rao (Academic Press, Orlando, 1985).

[14] A.R. Hoy, I.M. Mills, and G. Strey, Mol. Phys. 24, 1265 (1972).

[15] E. Hill and J.H. Van Vleck, Phys. Rev. 32, 250 (1928).

[16] J.H. Van Vleck, Phys. Rev. 33, 467 (1929).

[17] L. Pauling and E.B. Wilson, Jr., Introduction to Quantum Mechanics (Dover, New York, 1985).

[18] H. Lefebvre and R.W. Field, Perturbations in the Spectra of Diatomic Molecules (Academic Press, London, 1986), pp. 139-143.

[19] R.N. Zare, Angular Momentum (Wiley, New York, 1988), pp. 279-281.

[20] R.W. Field, J.H. Baraban, S.H. Lipoff, and A.R. Beck, in Handbook of High-Resolution Spectroscopy, edited by M. Quack and F. Merkt (Wiley, New York, 2011).

[21] A.B. McCoy and E. L. Sibert III, J. Chem. Phys. 97 (5), 2938 (1992).

[22] T.L. Chow, Mathematical Methods for Physicists (Cambridge University Press, Cambridge, 2000), pp. 452-453.

[23] E.B. Wilson, Jr., J.C. Decius, and P.C. Cross, Molecular Vibrations (Dover, New York, 1955/1980), p. 284.

[24] D. Huber, Int. J. Quantum Chem. 28, 245 (1985).

[25] G. Herzberg, Molecular Spectra and Molecular Structure, vol. II (Van Nostrand Reinhold Company, New York, 1945).

[26] W.F. Polik and J. R. van Ommen, in Highly Excited States: Relaxation, Reactions, and Structure, edited by A.S. Mullin and G.C. Schatz (American Chemical Society, Washington D.C., 1997), pp. 51-68.

[27] S.V.Krasnoshchekov, N.C. Craig, and N.F. Stepanov, J. Chem. Phys. A 117 (14), 3041 (2013).

[28] M.M. Law, Ph.D. Thesis, University of Aberdeen, 1993 (unpublished). 
[29] E. Fermi, Z. Phys. 71, 250 (1931).

[30] B.T. Darling and D.M. Dennison, Phys. Rev. 57, 128 (1940).

[31] J.L. Davisson, N.R. Brinkmann, and W.F. Polik, Mol. Phys. 110 (19-20), 2587 (2012).

[32] M.S. Schuurman, W.D. Allen, and H.F. Schaefer III, J. Comp. Chem. 26 (11), 1106 (2005).

[33] V. Barone, J. Chem. Phys. 122, 14108 (2005).

[34] M. J. Frisch, G.W. Trucks, H.B. Schlegel, G.E. Scuseria, and others, Gaussian09, Revision A.2, Gaussian, Inc., Wallingford, CT, 2009.

[35] J. Bloino, M. Biczysko, and V. Barone, J. Chem. Theory Comput. 8, 1015 (2012).

[36] C.Y. Lin, A.T.B. Gilbert, P.M.W. Gill, Theor. Chem. Account. 120, 23 (2008).

[37] J.F. Stanton, J. Gauss, M.E. Harding, P.G. Szalay, and others, CFOUR, $<$ www.cfour.de $>$.

[38] S.A.B. Solina, J.P. O’Brien, R.W. Field, and W.F. Polik, J. Phys. Chem. 100, 7797 (1996).

[39] C. Mukarakate, C. Tao, C. D. Jordan, W. F. Polik, and S. A. Reid, J. Phys. Chem. 112, 466 (2008).

[40] Free Software Foundation, GCC Compiler Collection (Free Software Foundation, Inc., Boston, MA, 2013).

[41] J.F. Gaw, A. Willets, W.H. Green and N.C. Handy, in Advances in Molecular Vibrations and Collision Dynamics, edited by J.M. Bowman (JAI Press, Greenwich, 1990), p. 169, SPECTRO.

[42] R.J. Bouwens, J.A. Hammerschmidt, T.A. Stegink, M.M. Grzeskowiak, P.M. Yorba, and W.F. Polik, J. Chem. Phys. 104, 460 (1996).

[43] K.K. Ellsworth, B.D. Lajiness, J.P. Lajiness, and W.F. Polik, J. Mol. Spec. 252, 205 (2008).

[44] K.K. Ellsworth, J.D. Herdman, B.D. Lajiness, J.P. Lajiness, and W.F. Polik (preprint available).

[45] G.D. Martin, T.P. Chassee, W.F. Polik (in preparation).

[46] D.C. Burleigh and E.L. Sibert III, J. Chem. Phys. 98 (11), 8419 (1993). 
Table 1. Harmonic oscillator basis function matrix elements [1-2].

\begin{tabular}{|l|l|}
\hline$\left\langle\mathrm{v}_{i}\left|q_{i}\right| \mathrm{v}_{i}+1\right\rangle=\left(\frac{\mathrm{v}_{i}+1}{2}\right)^{\frac{1}{2}}$ & $\left\langle\mathrm{v}_{i}\left|p_{i} q_{i}\right| \mathrm{v}_{i}\right\rangle=-\frac{1}{2} i$ \\
\hline$\left\langle\mathrm{v}_{i}\left|q_{i}\right| \mathrm{v}_{i}-1\right\rangle=\left(\frac{\mathrm{v}_{i}}{2}\right)^{\frac{1}{2}}$ & $\left\langle\mathrm{v}_{i}\left|p_{i}^{2}\right| \mathrm{v}_{i}\right\rangle=\left(\mathrm{v}_{i}+\frac{1}{2}\right)$ \\
\hline$\left\langle\mathrm{v}_{i}\left|p_{i}\right| \mathrm{v}_{i}+1\right\rangle=-i\left(\frac{\mathrm{v}_{i}+1}{2}\right)^{\frac{1}{2}}$ & $\left\langle\mathrm{v}_{i}\left|q_{i}^{3}\right| \mathrm{v}_{i}+3\right\rangle=\frac{1}{2}\left[\frac{\left(\mathrm{v}_{i}+1\right)\left(\mathrm{v}_{i}+2\right)\left(\mathrm{v}_{i}+3\right)}{2}\right]^{\frac{1}{2}}$ \\
\hline$\left.\left\langle\mathrm{v}_{i}\left|p_{i}\right| \mathrm{v}_{i}-1\right\rangle=i\left(\frac{\mathrm{v}_{i}}{2}\right)^{\frac{1}{2}}+1\right\rangle=\frac{3}{2}\left[\frac{\left(\mathrm{v}_{i}+1\right)^{3}}{2}\right]^{\frac{1}{2}}$ & $\left\langle\mathrm{v}_{i}\left|q_{i}^{3}\right| \mathrm{v}_{i}-1\right\rangle=\frac{3}{2}\left(\frac{\mathrm{v}_{i}^{3}}{2}\right)^{\frac{1}{2}}$ \\
\hline$\left.\left\langle\mathrm{v}_{i}\left|q_{i}^{2}\right| \mathrm{v}_{i}+2\right\rangle=\frac{1}{2}\left[\left(\mathrm{v}_{i}+1\right)\left(\mathrm{v}_{i}+2\right)\right]\right]^{\frac{1}{2}}$ & $\left\langle\mathrm{v}_{i}\left|q_{i}^{3}\right| \mathrm{v}_{i}-3\right\rangle=\frac{1}{2}\left[\frac{\mathrm{v}_{i}\left(\mathrm{v}_{i}-1\right)\left(\mathrm{v}_{i}-2\right)}{2}\right]^{\frac{1}{2}}$ \\
\hline$\left\langle\mathrm{v}_{i}\left|q_{i}^{2}\right| \mathrm{v}_{i}\right\rangle=\left(\mathrm{v}_{i}+\frac{1}{2}\right)$ & $\left\langle\mathrm{v}_{i}\left|q_{i}^{4}\right| \mathrm{v}_{i}\right\rangle=\frac{3}{2}\left[\left(\mathrm{v}_{i}+\frac{1}{2}\right)^{2}+\frac{1}{4}\right]$ \\
\hline$\left\langle\mathrm{v}_{i}\left|q_{i}^{2}\right| \mathrm{v}_{i}-2\right\rangle=\frac{1}{2}\left[\mathrm{v}_{i}\left(\mathrm{v}_{i}-1\right)\right]^{\frac{1}{2}}$ & \\
\hline$\left\langle\mathrm{v}_{i}\left|q_{i} p_{i}\right| \mathrm{v}_{i}\right\rangle=\frac{1}{2} i$ & \\
\hline
\end{tabular}




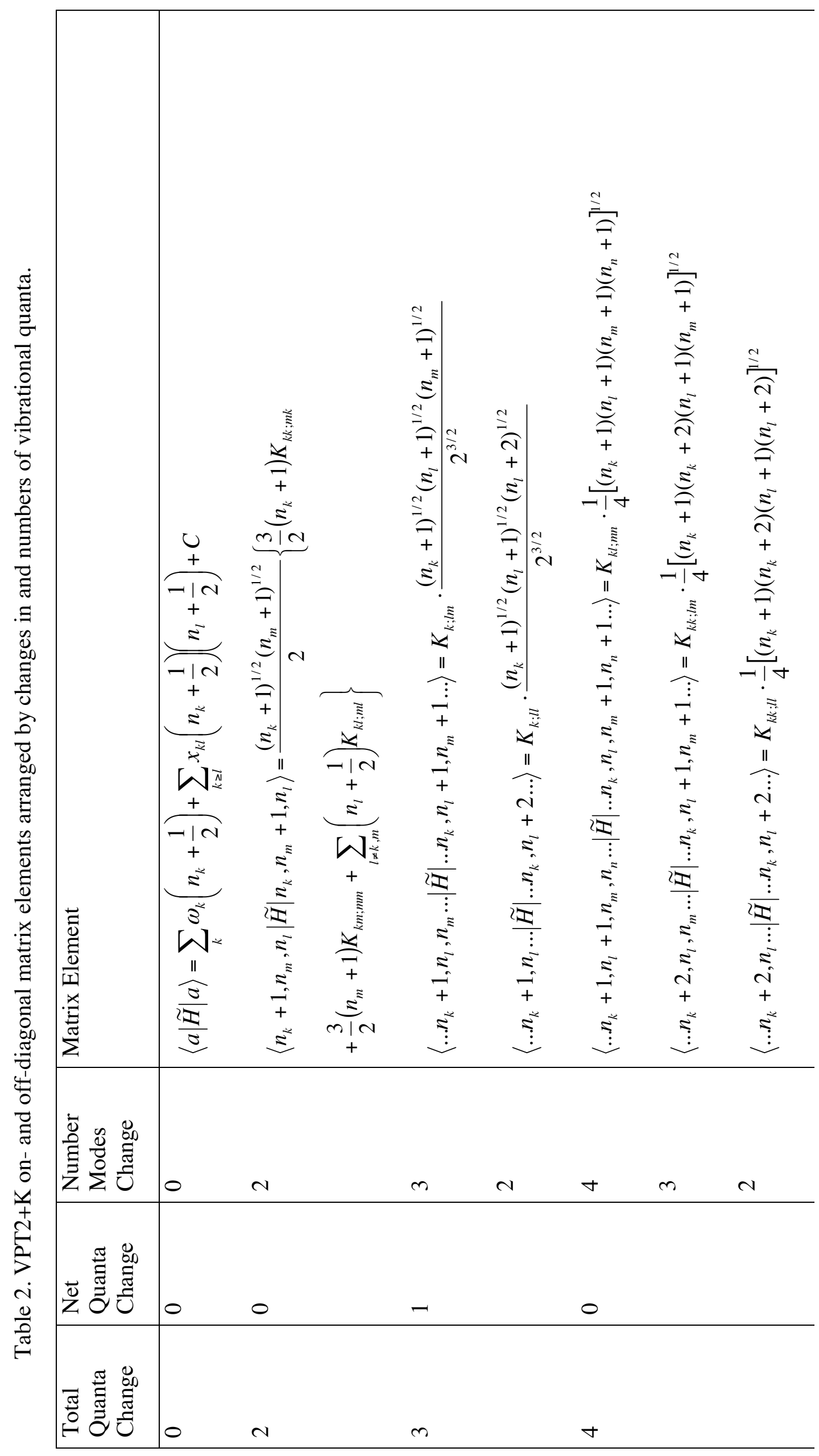




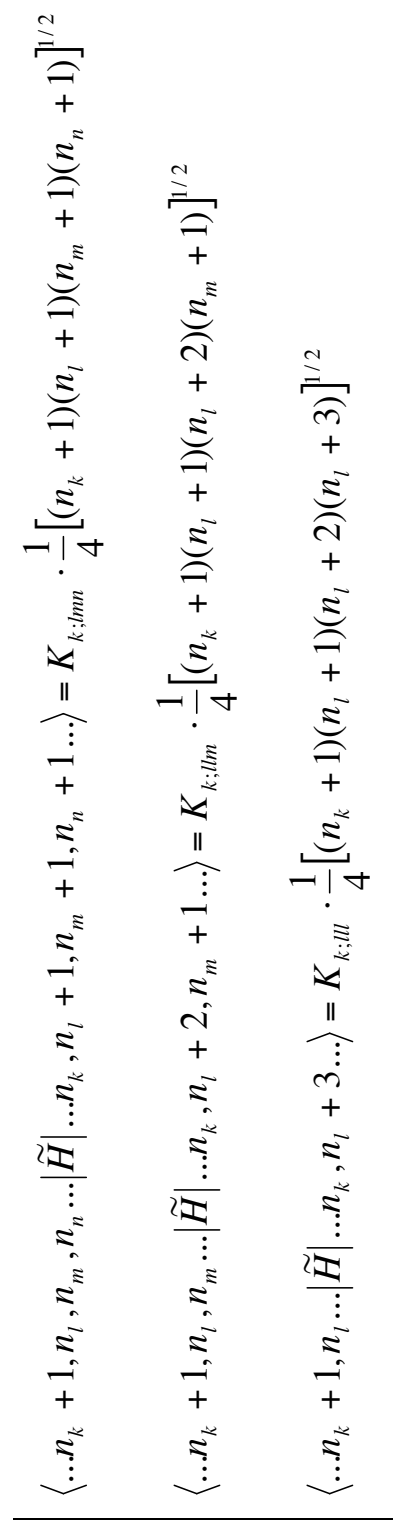

$\forall m \quad r$

N 


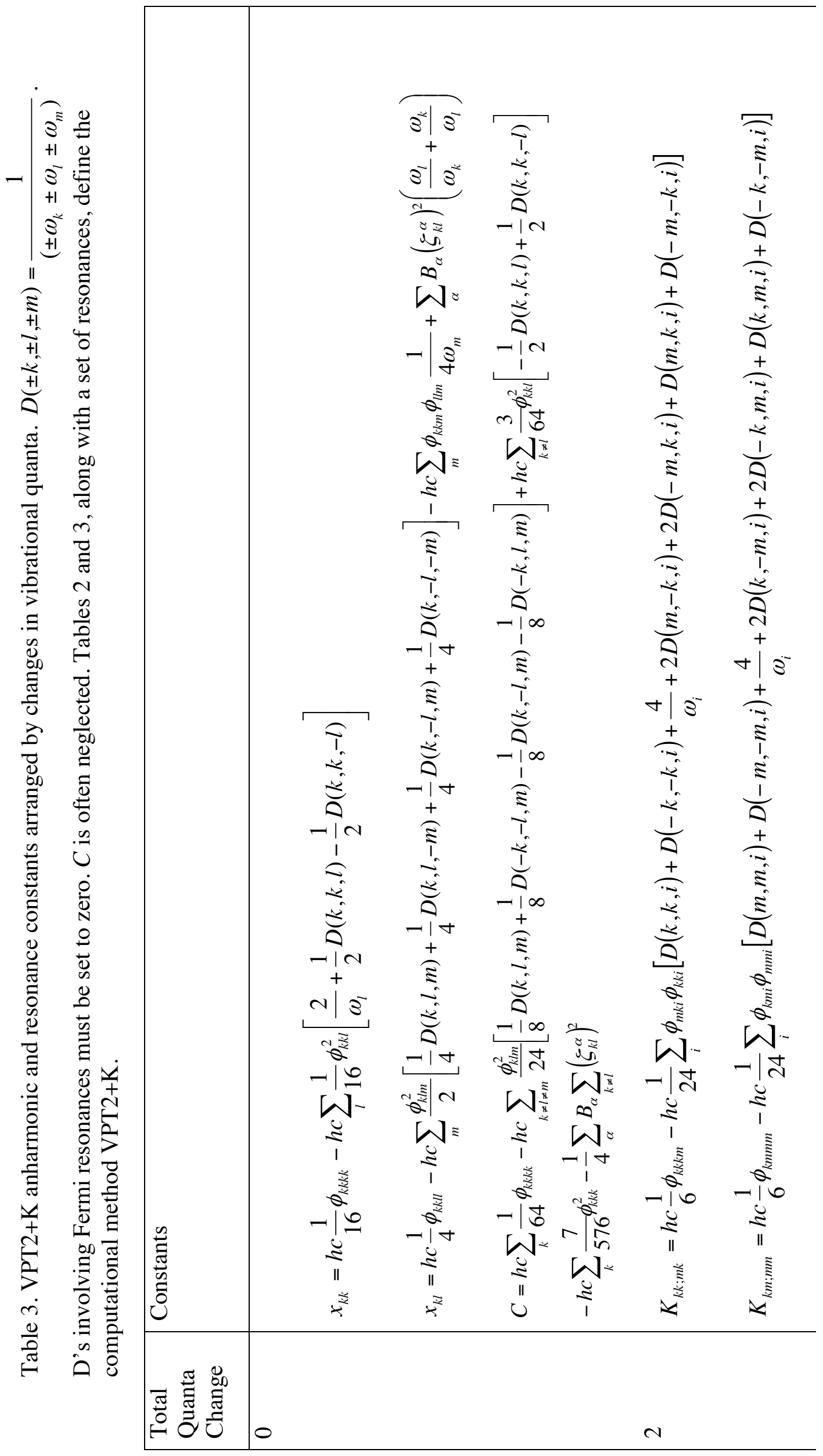




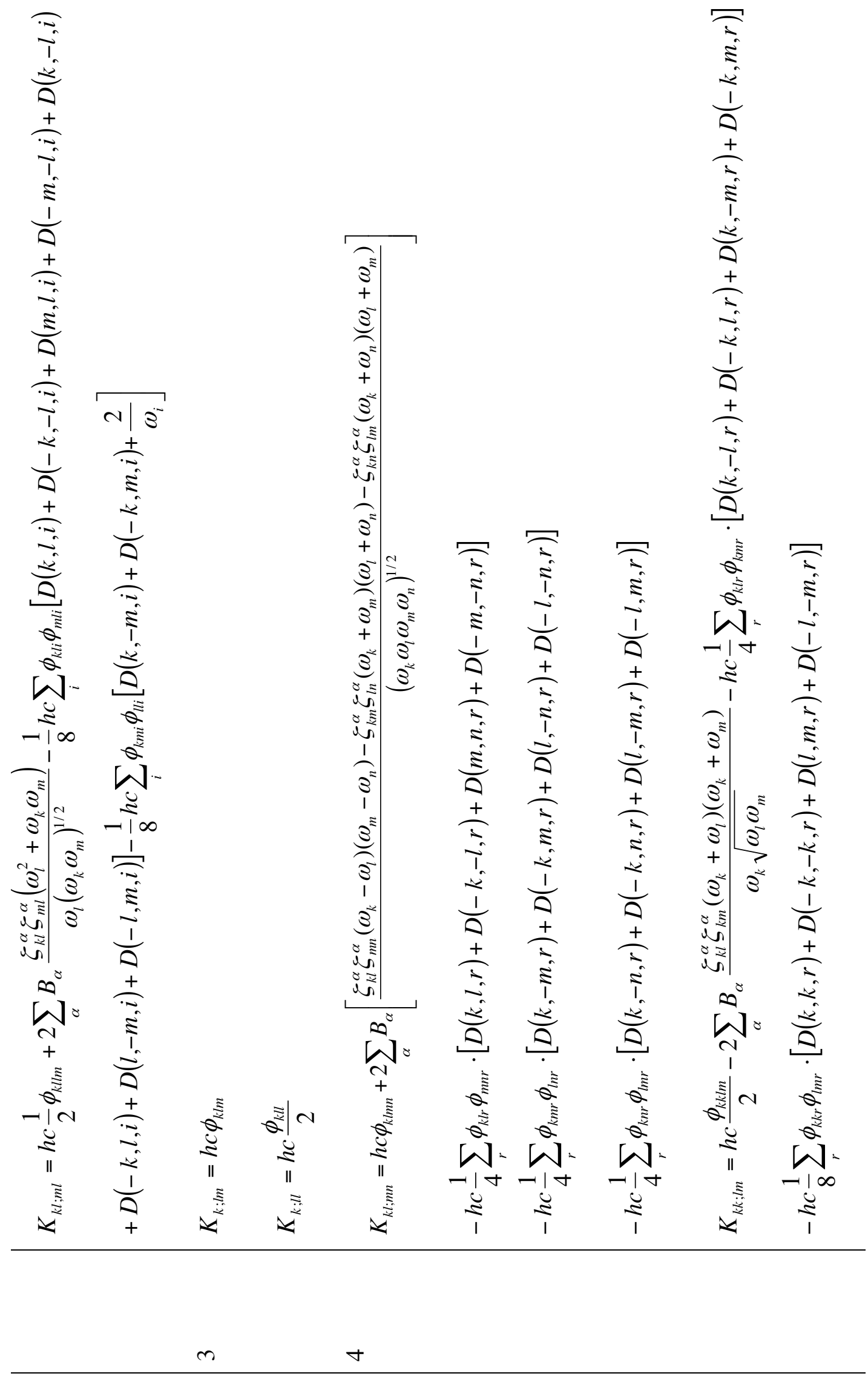




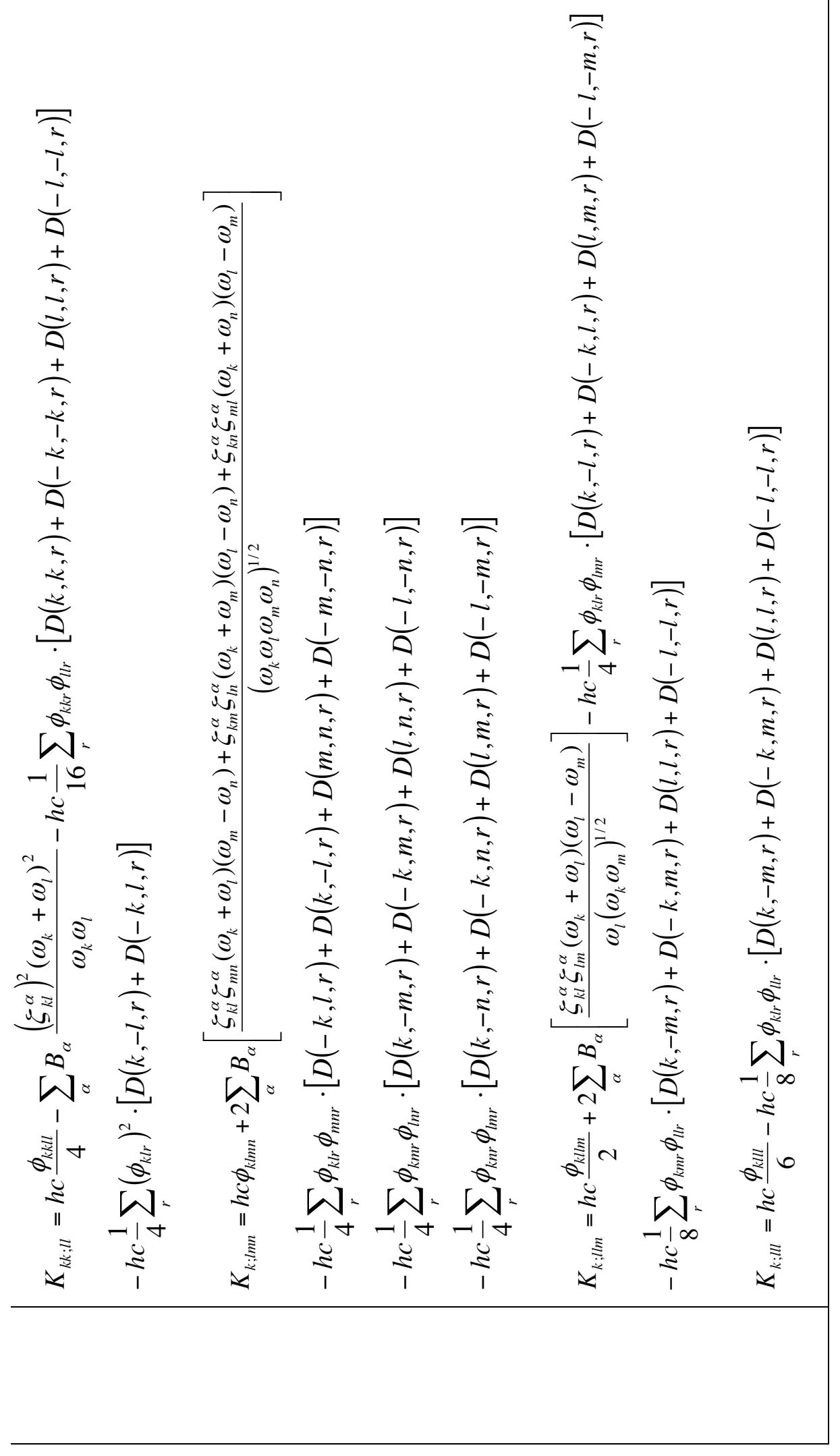


Table 4. Diagonal matrix elements for the $\langle 111111|$ state of formaldehyde. The cubic terms of $\widetilde{H}^{\prime \prime}$ are identified according to their order in Equation (66).

\begin{tabular}{|c|c|c|c|}
\hline Term & $\operatorname{HOME}\left(\mathrm{cm}^{-1}\right)$ & $\mathrm{E}\left(\mathrm{cm}^{-1}\right)$ & XKL $\left(\mathrm{cm}^{-1}\right)$ \\
\hline$\tilde{H}^{o}$ & 17551.5009 & 17551.5009 & 17551.5009 \\
\hline$\tilde{H}^{\prime}$ & 0 & & \\
\hline$\widetilde{H}^{\prime \prime}$ & -907.4029 & & \\
\hline Quartic & & -224.7133 & \\
\hline $3^{\text {rd }}$ Cubic & & 159.8452 & \\
\hline $4^{\text {th }}$ Cubic & & -188.4489 & \\
\hline $2^{\text {nd }}+11^{\text {th }}$ Cubic & & -196.2608 & \\
\hline $1^{\text {st }}+8^{\text {th }}+10^{\text {th }}$ Cubic & & -406.1208 & \\
\hline $5^{\text {th }}+6^{\text {th }}+7^{\text {th }}+9^{\text {th }}$ Cubic & & -122.1396 & \\
\hline Vibrational Angular Momentum & & 70.4353 & \\
\hline Total Anharmonicities & & & -932.2557 \\
\hline$+\mathrm{C}$ Terms & & & 24.8521 \\
\hline Total & 16644.0973 & 16644.0973 & 16644.0973 \\
\hline
\end{tabular}


Table 5. Off-diagonal matrix elements for formaldehyde for $K_{3 ; 56}, K_{44 ; 66}$, and $K_{2 ; 3}$.

\begin{tabular}{|c|c|c|c|c|c|c|}
\hline \multirow[t]{2}{*}{ Term } & \multicolumn{2}{|c|}{$\langle 002002|\tilde{H}| 001011\rangle$} & \multicolumn{2}{|c|}{$\langle 020200|\tilde{H}| 020002\rangle$} & \multicolumn{2}{|c|}{$\langle 002002|\tilde{H}| 011002\rangle$} \\
\hline & $\begin{array}{c}\text { HOME } \\
\left(\mathrm{cm}^{-1}\right)\end{array}$ & $\mathrm{E}\left(\mathrm{cm}^{-1}\right)$ & $\operatorname{HOME}\left(\mathrm{cm}^{-1}\right)$ & $\mathrm{E}\left(\mathrm{cm}^{-1}\right)$ & $\begin{array}{c}\mathrm{HOME} \\
\left(\mathrm{cm}^{-1}\right)\end{array}$ & $\mathrm{E}\left(\mathrm{cm}^{-1}\right)$ \\
\hline$\tilde{H}^{\prime}$ & 132.2493 & 132.2493 & 0 & 0 & 0 & 0 \\
\hline$\widetilde{H}^{\prime \prime}$ & & & & & & \\
\hline Quartic & 0 & 0 & 3.7737 & 3.7737 & 17.2513 & 17.2513 \\
\hline Cubic & 0 & 0 & -11.7194 & -11.7194 & -22.0288 & -22.0288 \\
\hline $\begin{array}{c}\text { Vibrational } \\
\text { Angular } \\
\text { Momentum }\end{array}$ & 0 & 0 & -5.1933 & -5.1933 & -0.9012 & -0.9012 \\
\hline Total & 132.2493 & 132.2493 & -13.1391 & -13.1391 & -5.6787 & -5.6787 \\
\hline
\end{tabular}


Table 6. Vibrational energy levels for a polyad of states in $\mathrm{H}_{2} \mathrm{CO}$ considering the resonances $K_{5 ; 26}, K_{5 ; 36}, K_{44 ; 66}, K_{11 ; 55}$, and $K_{2 ; 3}$.

\begin{tabular}{|c|c|c|c|c|c|}
\hline State & $\begin{array}{c}\text { Experiment } \\
\left(\mathrm{cm}^{-1}\right)\end{array}$ & PT2 $\left(\mathrm{cm}^{-1}\right)$ & $\begin{array}{c}\text { PT2 }- \text { Exp } \\
\left(\mathrm{cm}^{-1}\right)\end{array}$ & $\begin{array}{c}\text { VPT2+K } \\
\left(\mathrm{cm}^{-1}\right)\end{array}$ & VPT2+K - Exp $\left(\mathrm{cm}^{-1}\right)$ \\
\hline $3_{2} 4_{2}$ & 5321.29 & 5323.53 & 2.24 & 5318.13 & -3.16 \\
$3_{2} 6_{2}$ & 5389.36 & 5407.82 & 18.46 & 5334.17 & -55.19 \\
$1_{2}$ & 5462.72 & 5492.69 & 29.97 & 5452.58 & -10.14 \\
$2_{1} 3_{1} 4_{2}$ & 5546.50 & 5547.16 & 0.66 & 5542.69 & -3.81 \\
$3_{1} 5_{1} 6_{1}$ & 5551.35 & 5401.23 & -150.12 & 5550.61 & -0.74 \\
$5_{2}$ & 5650.98 & 5468.57 & -182.41 & 5655.89 & 4.91 \\
$2{ }_{1} 3_{1} 6_{2}$ & 5687.89 & 5813.72 & 125.83 & 5683.59 & -4.30 \\
$2{ }_{2} 4_{2}$ & 5768.77 & 5766.96 & -1.81 & 5765.69 & -3.08 \\
$25_{1} 5_{1}$ & 5809.50 & 5671.38 & -138.12 & 5812.56 & 3.06 \\
$2{ }_{2} 6_{2}$ & 5986.19 & 6215.79 & 229.60 & 5994.12 & 7.93 \\
RMS Difference & & & 120.28 & & 18.16 \\
\hline
\end{tabular}


Table 7. Comparison of resonance constants $K_{2 i ; 3 i}$ for the 2-3 resonance in $\mathrm{H}_{2} \mathrm{CO}$.

\begin{tabular}{|c|c|}
\hline Resonance Constant & Constant Value $\left(\mathrm{cm}^{-1}\right)$ \\
\hline$K_{21 ; 31}$ & 32.6083 \\
$K_{22 ; 32}$ & -21.5924 \\
$K_{23 ; 33}$ & -10.1190 \\
$K_{24 ; 34}$ & -3.0937 \\
$K_{25 ; 35}$ & 36.9339 \\
$K_{26 ; 36}$ & -8.1560 \\
\hline
\end{tabular}


Table 8. Vibrational energy levels for a polyad of states in HDCO considering the resonances $K_{5 ; 33}, K_{1 ; 44}, K_{1 ; 66}$, and $K_{44 ; 66}$.

\begin{tabular}{|c|c|c|c|c|c|}
\hline State & Experiment $\left(\mathrm{cm}^{-1}\right)$ & PT2 $\left(\mathrm{cm}^{-1}\right)$ & $\begin{array}{c}\text { PT2 }- \text { Exp } \\
\left(\mathrm{cm}^{-1}\right)\end{array}$ & VPT2 $+\mathrm{K}\left(\mathrm{cm}^{-1}\right)$ & VPT2+K - Exp $\left(\mathrm{cm}^{-1}\right)$ \\
\hline $1_{1} 4_{1} 6_{2}$ & 5067.90 & 5239.26 & 171.36 & 5036.94 & -30.96 \\
$1_{2} 4_{1}$ & 5150.15 & 5263.99 & 113.84 & 5118.19 & -31.96 \\
$1_{1} 4_{3}$ & 5189.75 & 5296.26 & 106.51 & 5185.94 & -3.81 \\
$4_{1} 6_{4}$ & 5231.32 & 5100.52 & -130.80 & 5233.76 & 2.44 \\
$4_{3} 6_{2}$ & 5252.28 & 5171.97 & -80.31 & 5273.06 & 20.78 \\
$4_{5}$ & 5318.63 & 5111.41 & -207.22 & 5335.51 & 16.88 \\
RMS & & & 141.52 & & 21.28 \\
Difference & & & & \\
\hline
\end{tabular}


Table 9. Vibrational energy levels for a polyad of states in $\mathrm{D}_{2} \mathrm{CO}$ considering the resonances $K_{5 ; 36}, K_{1 ; 44}, K_{1 ; 66}$, and $K_{44 ; 66}$.

\begin{tabular}{|c|c|c|c|c|c|}
\hline State & Experiment $\left(\mathrm{cm}^{-1}\right)$ & PT2 $\left(\mathrm{cm}^{-1}\right)$ & $\begin{array}{c}\text { PT2 }- \text { Exp } \\
\left(\mathrm{cm}^{-1}\right)\end{array}$ & VPT2+K $\left(\mathrm{cm}^{-1}\right)$ & VPT2+K $-\mathrm{Exp}_{\left(\mathrm{cm}^{-1}\right)}$ \\
\hline $3_{1} 4_{2} 6_{1}$ & 3937.59 & 3947.39 & 9.80 & 3920.21 & -17.38 \\
$3_{1} 6_{3}$ & 4014.81 & 4010.37 & -4.44 & 3986.73 & -28.08 \\
$4_{2} 5_{1}$ & 4007.91 & 4001.08 & -6.83 & 4010.06 & 2.15 \\
$1_{1} 3_{1} 6_{1}$ & - & 4103.47 & - & 4098.78 & - \\
$5_{1} 6_{2}$ & - & 4119.45 & - & 4130.96 & 9.46 \\
$1_{1} 5_{1}$ & 4172.14 & 4146.57 & -25.57 & 4181.60 & 17.21 \\
RMS & & & 14.28 & & \\
Difference & & & & & \\
\hline
\end{tabular}

